\title{
Biointerface Research in Applied Chemistry: Celebrating the Publication of 600 Papers in 2021, and First Bibliometric Analysis from 2016-2020
}

\author{
Waseem Hassan 1,* (D), Mehreen Zafar ${ }^{1(\mathbb{D})}$, Alexandru Mihai Grumezescu ${ }^{2,3}$ (D) \\ 1 Institute of Chemical Sciences, University of Peshawar, Peshawar 25120, Khyber Pakhtunkhwa, Pakistan \\ 2 Department of Science and Engineering of Oxide Materials and Nanomaterials, Faculty of Applied Chemistry and \\ Materials Science, Politehnica University of Bucharest, Romania \\ 3 Research Institute of the University of Bucharest (ICUB), University of Bucharest, Romania \\ * Correspondence: waseem_anw@yahoo.com (W.H);
}

Scopus Author ID 25651506900

Received: 15.03.2021; Revised: 5.04.2021; Accepted: 10.04.2021; Published: 17.04.2021

\begin{abstract}
Web of Science (WoS) is covering Biointerface Research in Applied Chemistry (BRIAC) from 2015 onwards. However, we performed its $1^{\text {st }}$ bibliometric analysis of the last five years (from 2016 to 2020). The data from WoS was retrieved on $8^{\text {th }}$ February 2021. In this period, BRIAC has published seven hundred and fifty-seven documents $(\mathrm{n}=757)$, majorly comprising research articles $(n=665)$, reviews $(n=86)$, and editorials $(n=6)$. In all publications, exactly 2519 authors, 838 institutes or departments, and 77 countries have contributed. For numerical representations, we designed the publication and citation clubs. One thousand four hundred and thirty-four $(n=1434)$ citations were noted. The total h-index was found to be 11 , while the citation per item was recorded to be 1.89 . The unofficial impact factor is 1.93. The calculation is based on citations in 2020 (498), divided by papers published in 2018-2019 (258). By VOSviewer analysis, we also represented the co-authorship network for authors, institutes, and countries. Four thousand nine hundred and thirty-eight $(n=4938)$ keywords were noted in all publications. By co-words analysis, we represented the trends in publications. From WoS, we retrieved the per year h-index, citation per item, and total citations of BRIAC. It is worthy to note that BRIAC has accepted 600 research articles for the year 2021. All issues in 2021 are already published in advance. The present research article (bibliometric report) is paper number $600^{\text {th }}$, and it is closing the year. Based on the data, it can be concluded that in a short span of the last five years, BRIAC showed positive growth.
\end{abstract}

Keywords: Bibliometric Analysis; Web of Science; Co words Analysis Abbreviations: Web of Science (WoS), Biointerface Research in Applied Chemistry (BRIAC) Q4 = 4th Quartile, SJR= SCImago journal rank.

(C) 2021 by the authors. This article is an open-access article distributed under the terms and conditions of the Creative Commons Attribution (CC BY) license (https://creativecommons.org/licenses/by/4.0/).

\section{Introduction}

Bibliometrics is a quantitative and statistical analysis tool that illustrates the publication patterns related to a particular field or a source of publications. It offers a general outline and overall structure of a particular research area [1]. It can also evaluate and determine the influence and contributions of various journals, countries, and institutions [2]. The bibliometric analysis also helps identify the leading journals, authors, and countries involved in publications in a particular research area [3]. The present project is designed to study the publication history and progress of Biointerface Research in Applied Chemistry (BRIAC). The data was retrieved 
from the Web of Science (WoS) in February 2021. Based on the number of publications and citations, we aim to highlight the top-ranked researchers, institutions, and countries. We have also performed the co-words analysis, which may help understand the research trend or focus of publications.

\section{Materials and Methods}

\subsection{Source of information.}

In the present study, we used the WoS database. The data was retrieved in February 2021. We extracted the data from January 2016 to December 2020 using the name and ISSN of the journal (BRIAC). The authors collected, downloaded, and later quantitatively and qualitatively analyzed in Microsoft Excel 2013 for access type, year, author name, document type, keywords, affiliations, and country.

\subsection{Visualization maps.}

There are various software tools for conducting science mapping, for example, Bibexcel, Bibliometrix, BiblioMaps, CiteSpace, CitNetExplorer, SciMAT, and Sci ${ }^{2}$ Tool. Here, we used VOSviewer version 1.6.9. The software was developed by Van Eck and Waltman for constructing and visualizing bibliometric networks.

\section{Results and Discussion}

\subsection{Types of documents.}

BRIAC has completed and celebrated ten years of platinum open access [4]. WoS started covering it from 2015 onwards.

Per Year Publications

Total Citations

757

\begin{tabular}{|c|c|c|c|c|c|c|c|c|c|c|c|}
\hline 136 & 61 & 121 & 137 & 302 & $\begin{array}{c}757 \\
\end{array}$ & 294 & 125 & 351 & 367 & 297 & $\begin{array}{c}1434 \\
\bullet\end{array}$ \\
\hline$\approx$ & -1 & $\alpha$ & $\checkmark$ & & & 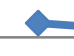 & 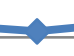 & $\leadsto$ & $\gamma$ & $\rightarrow$ & \\
\hline 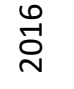 & 空 & $\stackrel{\infty}{\stackrel{1}{N}}$ & 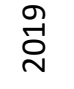 & 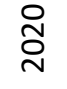 & 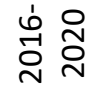 & $\begin{array}{l}\stackrel{0}{1} \\
\text { Dे }\end{array}$ & 穴 & $\stackrel{\infty}{\stackrel{1}{\sim}}$ & $\stackrel{\sigma}{\stackrel{N}{N}}$ & ণ్రి & 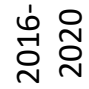 \\
\hline
\end{tabular}

h-Index

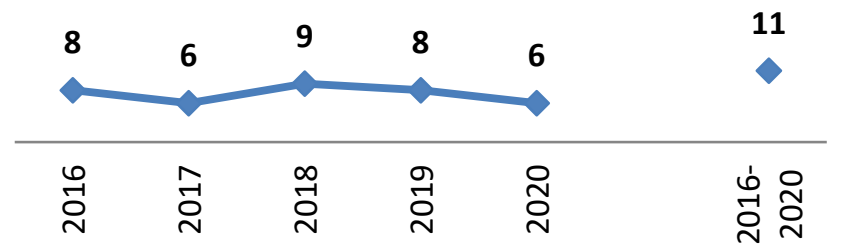

Citations Per Item

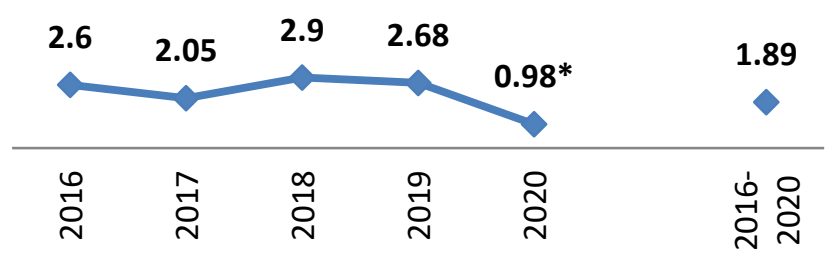

Figure 1. The per year publications, h-index, citations per item, and total citations obtained from WoS. (*=Most of the citations are not yet generated, and this is a normal value because the papers were just published, and there is a probable delay of 3-6 months until papers are cited. 
Till December 2020, it has completed research publications of seven hundred and fiftyseven documents $(n=757)$, majorly comprising of research articles $(n=665)$, reviews $(n=86)$, and editorials $(n=6)$. The total numbers of publications are described in Figure 1. The highest number of documents were published in 2020 ( $\mathrm{n}=302)$, followed by $2019(\mathrm{n}=137), 2016$ $(\mathrm{n}=136), 2018(\mathrm{n}=121)$ and $2017(\mathrm{n}=61)$. WoS categorized all publications in applied chemistry. Based on WoS data, we also presented the per year h-index, citations per document and total citations (Figure 1).

\subsection{Details about authors, institutes, \& countries and VOSviewer analysis.}

This part of the manuscript is dedicated to all researchers, institutions, and countries that have significantly contributed to all research publications $(n=757)$. Numerically we focused on a number of publications and citation analysis. It is a bibliometric technique that explores the patterns, frequency of citations, and academic researchers' efficiency [1-3]. Citation analysis can be carried out for authors, institutes, countries, and the most influential or productive documents. In all publications, exactly 2519 authors, 838 institutes, and 77 countries have significantly contributed (from 2016 till 2020). To categorize authors, institutes, and countries, we designed the publication and citation clubs. The data is depicted in Table 1 and Table 2, respectively. Furthermore, details about each author (Table S1), institute (Table S2), and country (Table S3) are also described. Based on the number of publications and total citations, we represented the top ten $(n=10)$ authors, institutes, and countries in Figure 2 A-E.

Table 1. Publication clubs (PC) for authors, institutes, and countries. NoA=Number of authors, NoI=Number of institutes, and $\mathrm{NoC}=$ Number of Countries.

\begin{tabular}{l|l|l|l|l|l}
$\mathbf{P C}$ & NoA & $\mathbf{P C}$ & NoI & PC & NoC \\
\hline 24 & 1 & 70 & 1 & $150-165$ & 2 \\
\hline 18 & 1 & 40 & 1 & $70-75$ & 1 \\
\hline 6 & 3 & $20-26$ & 2 & 30 to 39 & 6 \\
\hline 15 & 1 & 10 to 18 & 11 & 20 to 29 & 5 \\
\hline 14 & 2 & 9 & 4 & 10 to 16 & 5 \\
\hline 13 & 2 & 8 & 4 & 8 & 2 \\
\hline 12 & 1 & 7 & 3 & 7 & 3 \\
\hline 9 & 3 & 6 & 5 & 6 & 1 \\
\hline 8 & 5 & 5 & 17 & 5 & 4 \\
\hline 7 & 6 & 4 & 24 & 4 & 5 \\
\hline 6 & 6 & 3 & 55 & 3 & 7 \\
\hline 5 & 26 & 2 & 114 & 2 & 9 \\
\hline 4 & 33 & 1 & 597 & 1 & 27 \\
\hline 3 & 80 & Total & 838 & Total & 77 \\
\hline 2 & 263 & & & &
\end{tabular}

Table 2. Citations clubs (CC) for authors, institutes, and countries. NoA=Number of authors, NoI=Number of institutes, and $\mathrm{NoC}=$ Number of Countries.

\begin{tabular}{l|l|l|l|l|l}
$\mathbf{C C}$ & NoA & CC & NoI & CC & NoC \\
\hline 70 & 1 & 200 to 230 & 1 & 400 to 420 & 1 \\
\hline 60 & 1 & 100 to 160 & 2 & 200 to 300 & 2
\end{tabular}




\begin{tabular}{|c|c|c|c|c|c|}
\hline $\mathrm{CC}$ & NoA & $\mathrm{CC}$ & NoI & $\mathrm{CC}$ & NoC \\
\hline 50 to 60 & 3 & 40 to 90 & 5 & 50 to 90 & 6 \\
\hline 30 to 49 & 7 & 20 to 40 & 15 & 20 to 49 & 7 \\
\hline 20 to 29 & 18 & 10 to 19 & 50 & 10 to 19 & 10 \\
\hline 19 & 8 & 9 & 8 & 9 & 1 \\
\hline 18 & 5 & 8 & 13 & 8 & 5 \\
\hline 17 & 3 & 7 & 27 & 7 & 2 \\
\hline 16 & 11 & 6 & 24 & 6 & 4 \\
\hline 15 & 4 & 5 & 23 & 5 & 4 \\
\hline 14 & 3 & 4 & 44 & 4 & 4 \\
\hline 13 & 14 & 3 & 72 & 3 & 5 \\
\hline 12 & 8 & 2 & 84 & 2 & 1 \\
\hline 11 & 19 & 1 & 192 & 1 & 8 \\
\hline 10 & 33 & 0 & 278 & 0 & 17 \\
\hline 9 & 31 & Total & 838 & Total & 77 \\
\hline 8 & 43 & & & & \\
\hline 7 & 51 & & & & \\
\hline 6 & 71 & & & & \\
\hline 5 & 78 & & & & \\
\hline 4 & 112 & & & & \\
\hline 3 & 186 & & & & \\
\hline 2 & 259 & & & & \\
\hline 1 & 581 & & & & \\
\hline 0 & 969 & & & & \\
\hline Total & 2519 & & & & \\
\hline
\end{tabular}

\subsection{The VOSviewer analysis.}

Considerable literature is available, which confirms the importance of analysis of coauthorship, citations, and co-citation networks etc. We presented the results as "science mapping". The details of the analysis are given below. Co-author analysis consists of cooccurrences of authors, institutions, and countries [5]. In fact, it helps in understanding and evaluating scientific collaboration. Co-authorship analysis identifies the co-operation via research articles that are signed by at least two different researchers.

It can measure the volume of work carried out by the teams of scientists at the individual or institutional level and at the national or international level. It is a parameter for measuring the growth (or decline) of co-operative research as compared with research undertaken by a single scientist [6]. The foremost benefits of co-author analysis are endorsing access to modern and expensive instrumentations and assimilating worldwide researchers' participation in intricate tasks. Moreover, it also shows affirmative results on productivity, knowledge, and economic development [7]. Co-author analysis is considered a powerful method used for the identification of leading authors, organizations, and countries in a particular research field. To draw the co-authorship network in VOSviewer, we defined the minimum number of published articles to be three $(n=3)$ with zero $(n=0)$ citations. 170 authors were found to meet the threshold. The institutional co-authorship network is shown in Figure 3. 127 institutes have published at least three documents (Figure 4). 
NoP
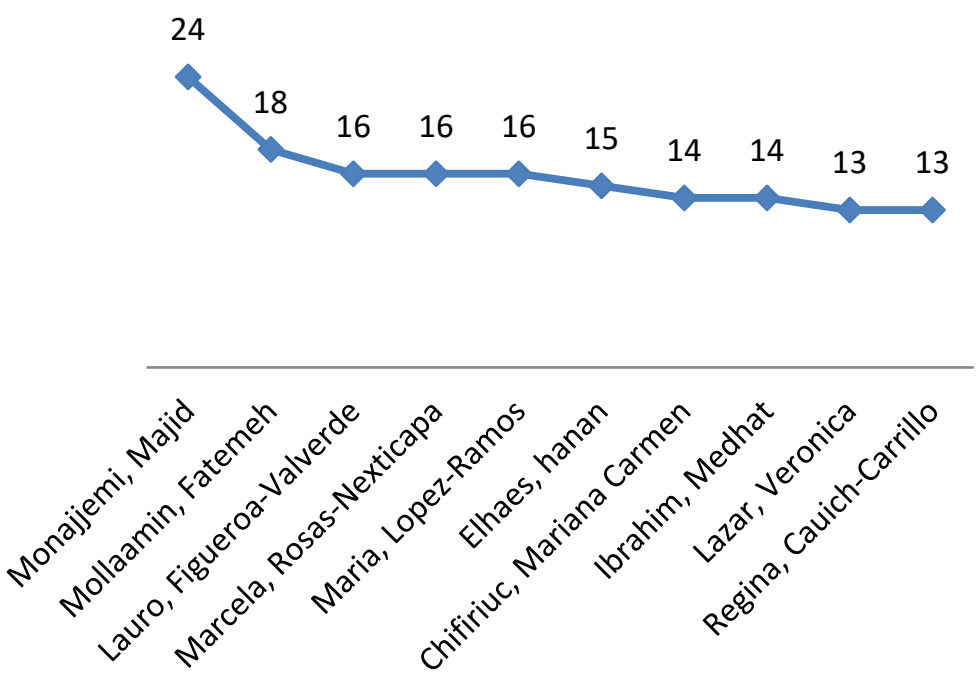

A (Authors)

NoD
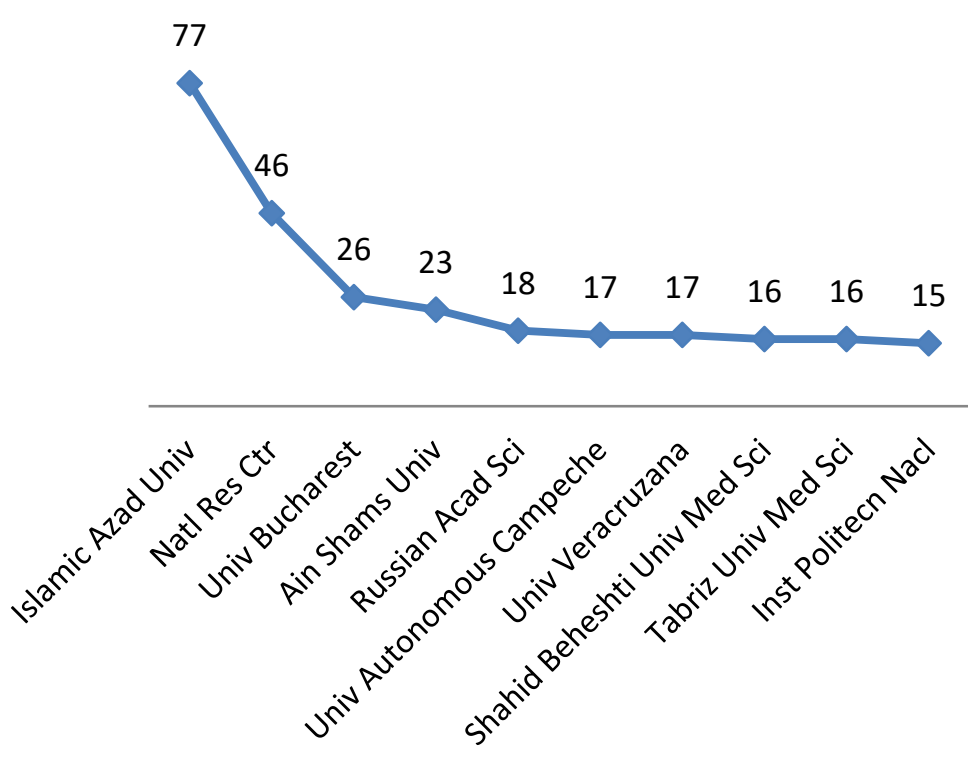

C (Institutes/Universities)

NoD

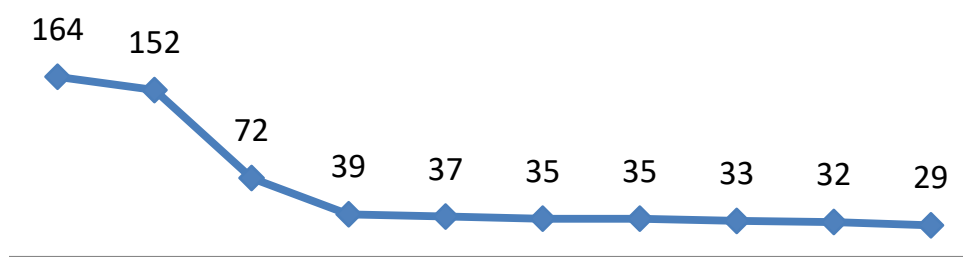

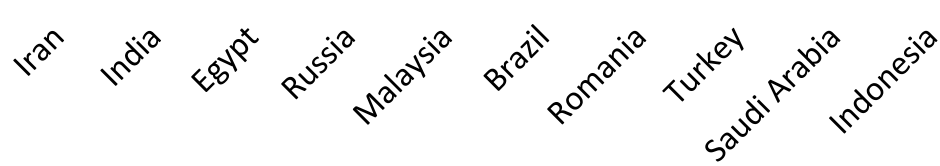

E (Countries)

Figure 2 (A-E). The top ten authors (A/B), institutes (C/D), and countries (E/F). Nop=number of publications and $\mathrm{NoC}=$ number of citations .

NoC

73

\section{B (Authors)}

NoC

226

D (Institutes/Universities)

NoC

\section{F (Countries)}
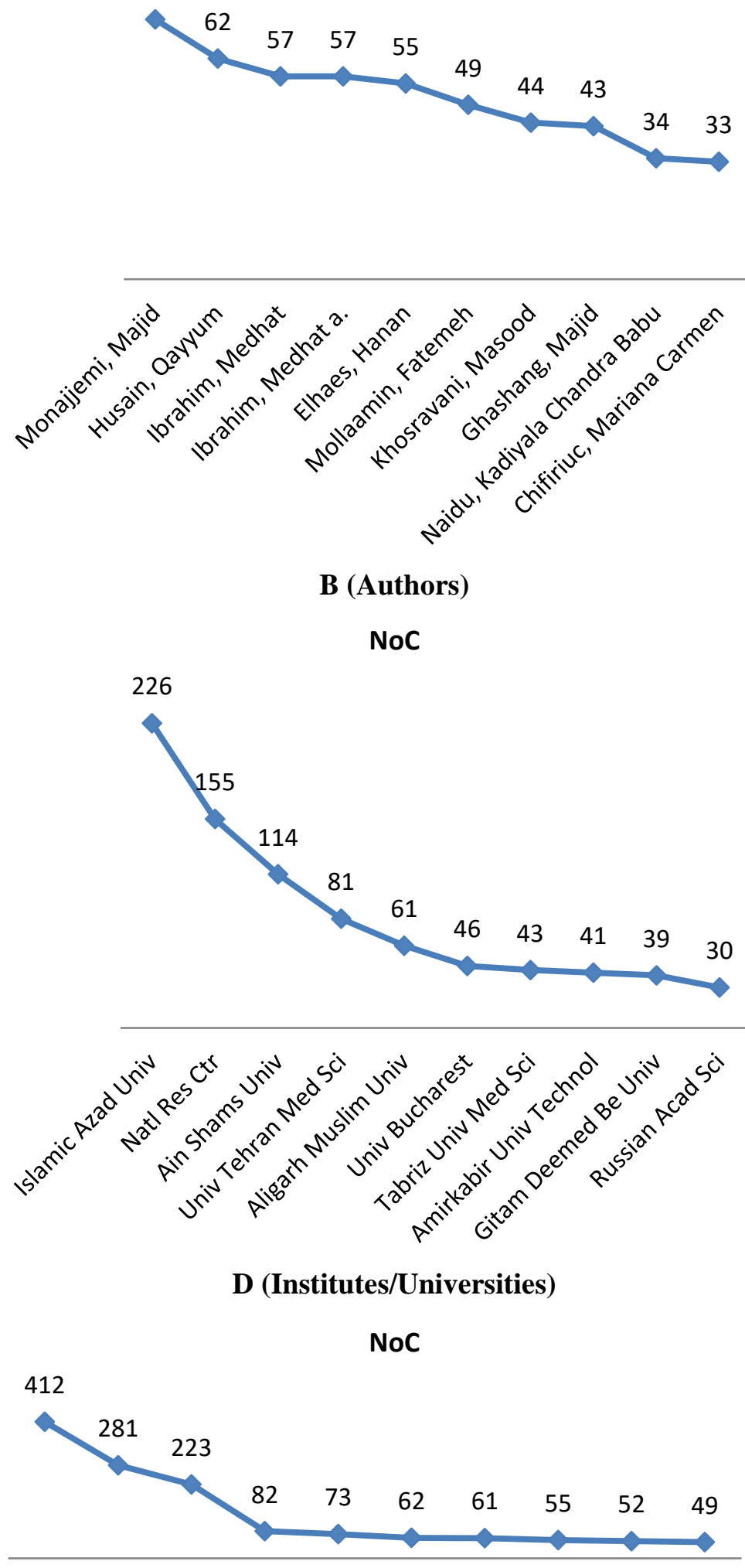

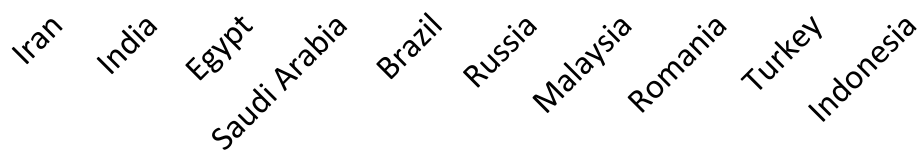

and $\mathrm{NoC}=$ number of citations. 

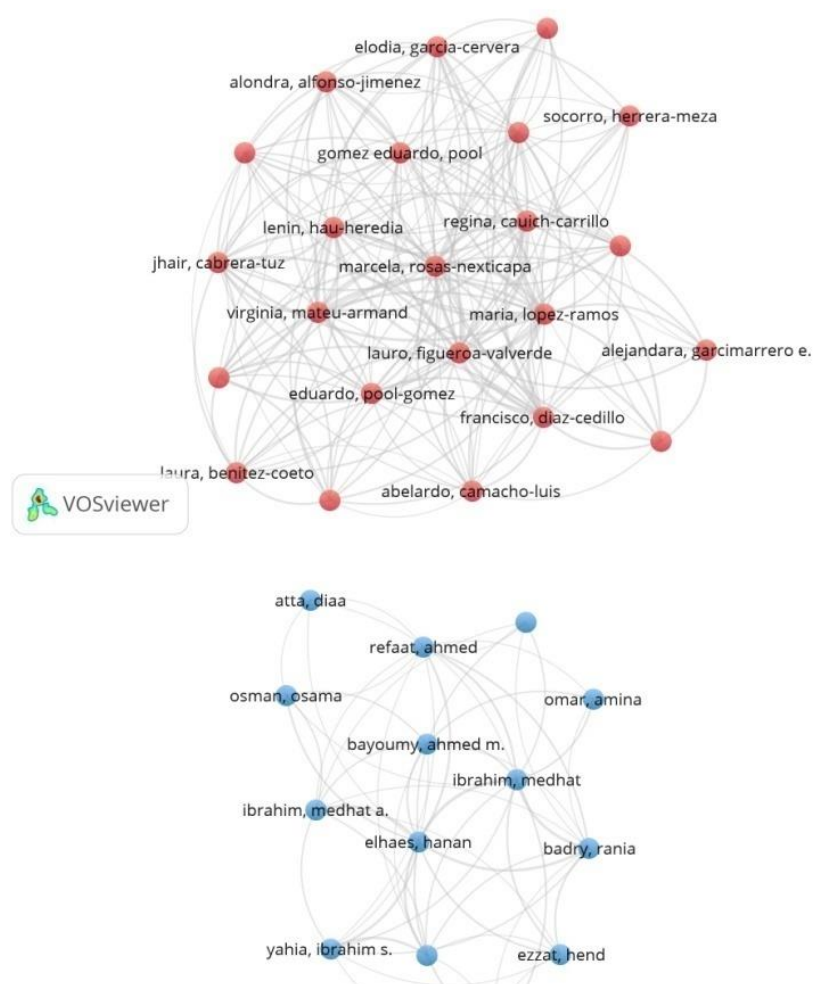

\& Vosviewer
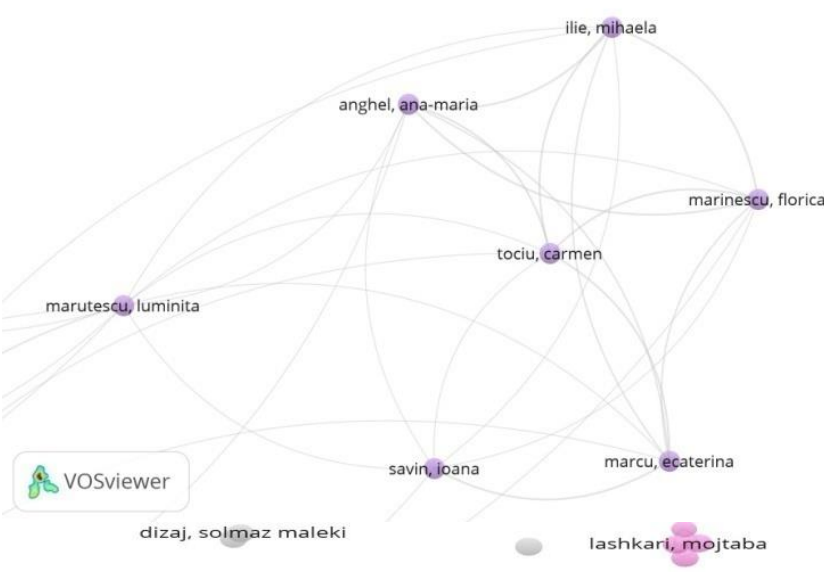

denizli, adil

al-anber, mohammed a.

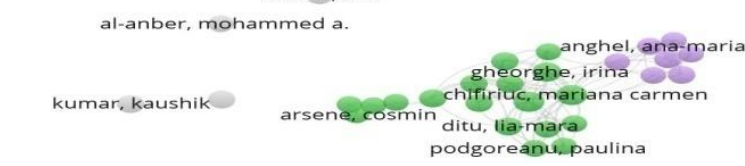

chaib, messaoud

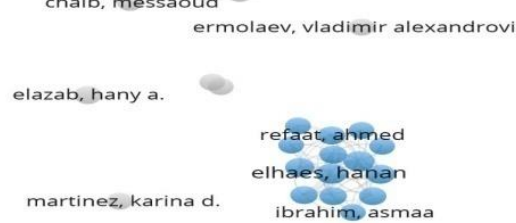

singh, inderbir

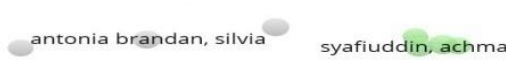

foroughifar, naser

elodia, garcia-cervera

gomez eduardo, pool

mafcela, rosas-nexticapa
lauro, figueroa-valverde

Sohrabvandi, sara

- maria virginia. mateu-armadbaltaci, abdulkerim kasim

\& vosviewer

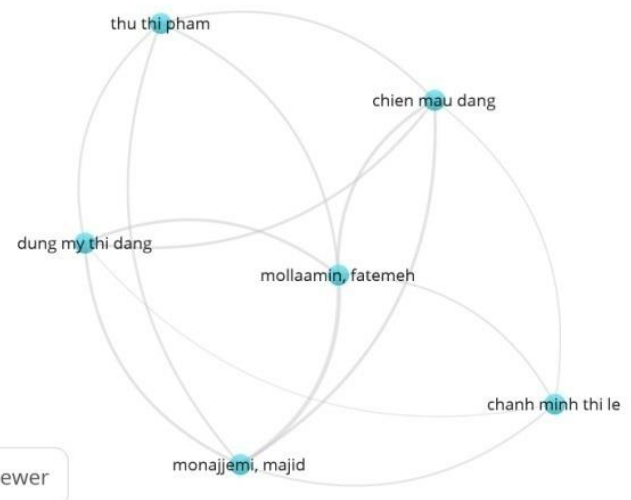

chien mau dang khalil, ahmed $\mathrm{m}$. monajjemi, majid

mandal, badal kumar

naidu, kadiyala chandra babu

naidu, $k$. chandra babu ningaiah, srikantamurthy manjunatha, $\mathrm{h}$.

curutiugarmen

cucu,natalia ionescubbogdan lazar, veronica

ratnam, $k$, venkata ratnamala, a.

pignatello, rosario bayraktar, oguz shah, munir h.

abdelghany, amr $\mathrm{m}$

fis vosviewer

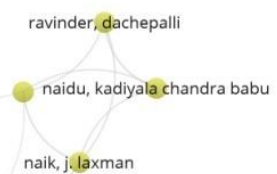

o, b. ramakrishna

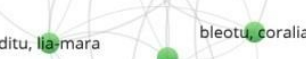

delcarus cristina

chifiriuc, mariana carmen

Figure 3. The co-authorship network for authors. 


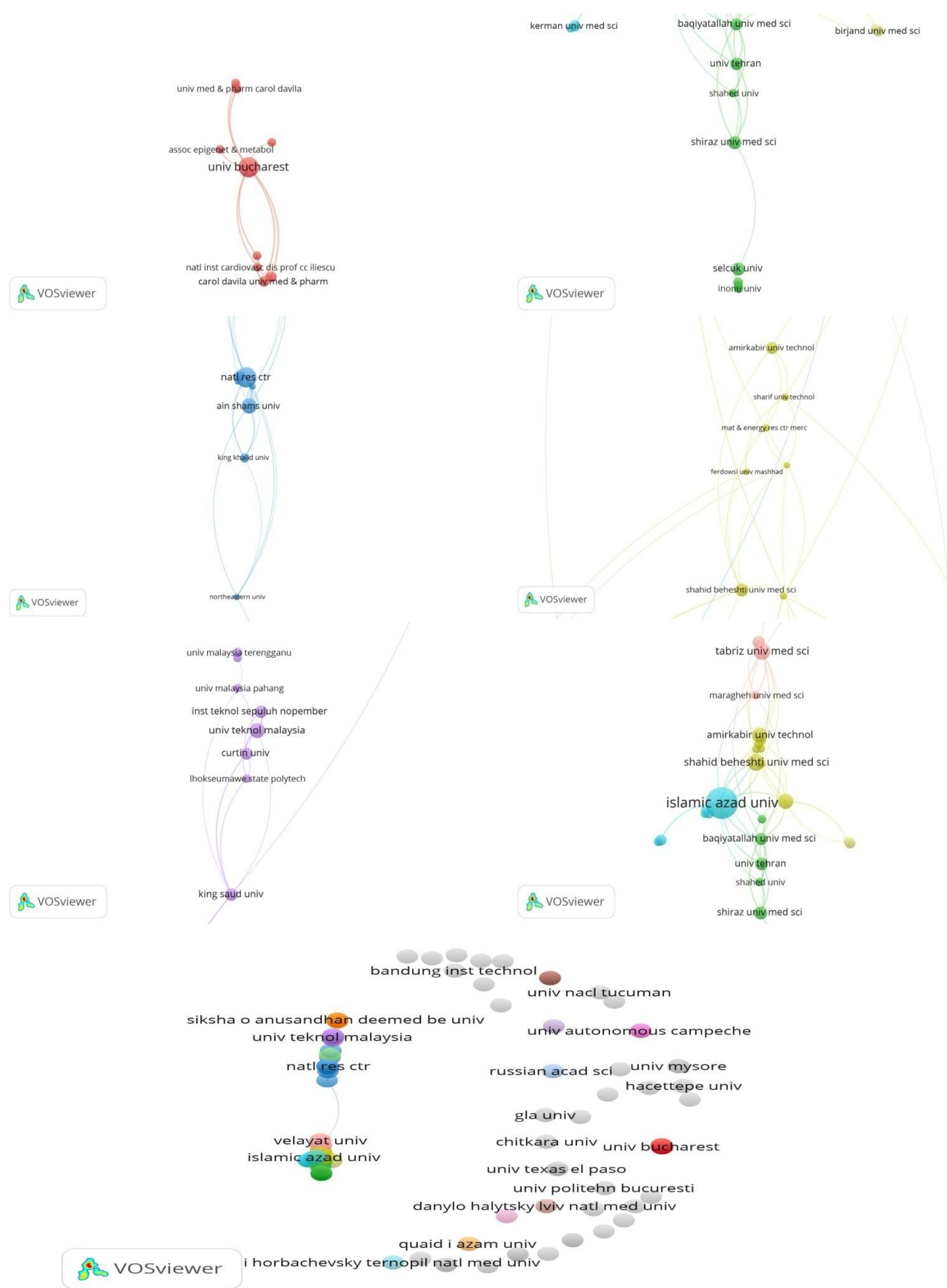

Figure 4. The co-authorship network for institutes. Different clusters are depicted. 


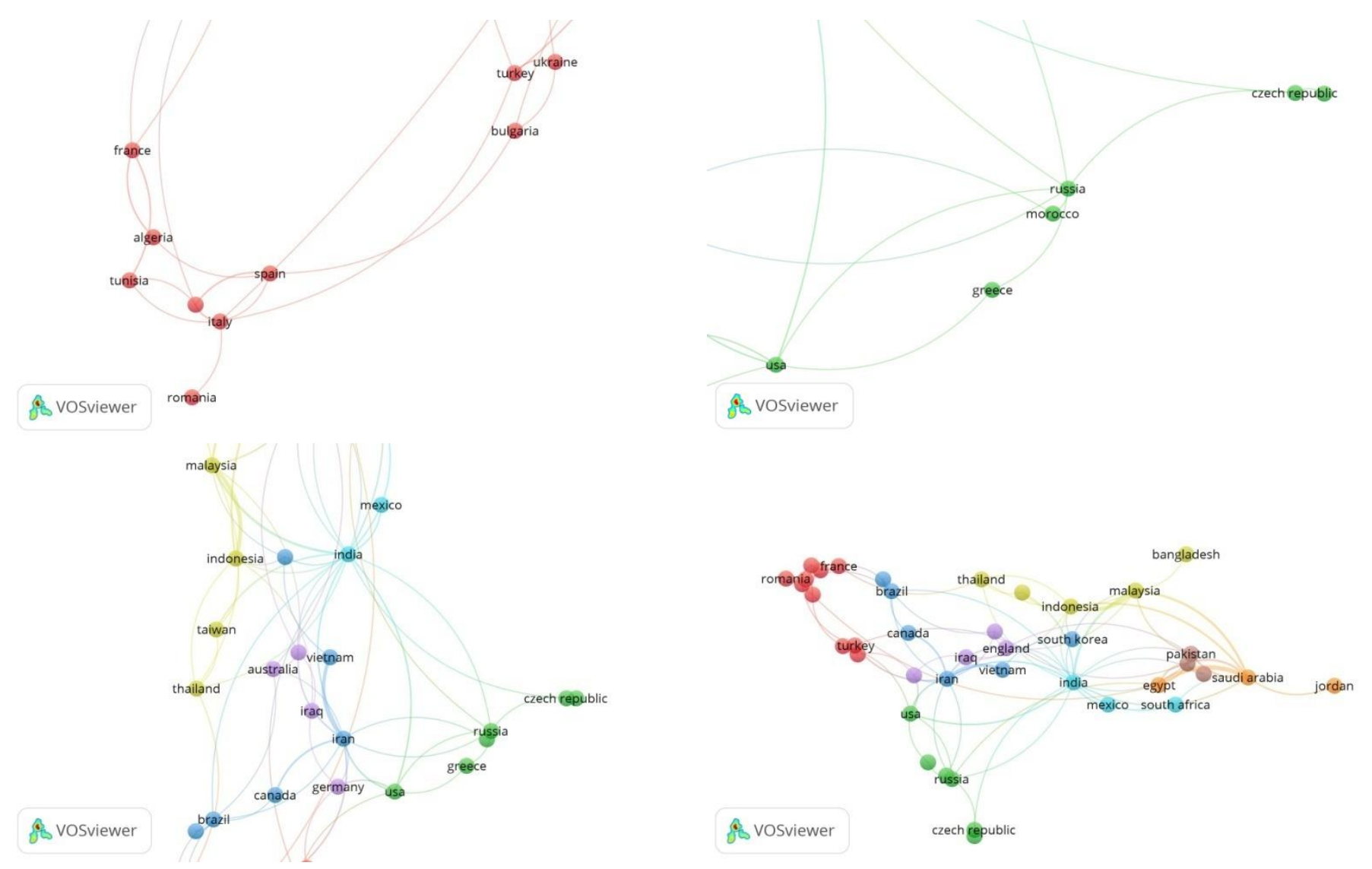

Figure 5. The co-authorship network for countries. Different clusters are depicted.

At the same time, 41 countries were found from the data, with at least three (3) publications and zero (0) citations. The data is presented in Figure 5.

\subsection{Co-word analysis.}

Co-words analysis is used to depict the main theme of a particular research area or a journal. It's important to note that more than four thousand nine hundred and thirty-eight $(n=4938)$ keywords appeared in all publications. 221 words appeared at least 5 times. However, we ignored simple words like animals, humans, rats, cells, etc. This may help in portraying the major research focus of all publications.

The publications focused on nanocomposites, nanofibers, nanomaterials, nanoparticles, nanotechnology, silver nanoparticles, magnetic nanoparticles, or different drugs, chemical or compounds like activated carbon, ascorbic acid, biomaterials, biosensor, chitosan, curcumin, essential oil, graphene, heavy metals, Ibuprofen, indigo carmine, pyrazole, and phenol. The authors studied their green chemistry, green synthesis, green method, or organic synthesis. Different instrumental techniques were applied for their characterizations, for example, XPS, XRD, TDM, vibrational analysis, ultrasonic velocity, FTIR, cyclic voltammetry, differential pulse voltammetry. The electrospinning, magnetic properties, and mechanical properties were also described. Furthermore, the authors also applied molecular docking, molecular dynamics simulation, molecular modeling, molecular structure, DFT calculations, docking, force fields, Freundlich to describe their geometrical characteristics or properties. Similarly, other keywords like catalytic behavior, cell membrane capacitors, degradation, dissolution, drug delivery, lithium-ion battery, photocatalysis, wastewater, water treatment, and adsorption may describe their chemical applications. While in biological applications, the authors studied oxidative stress, anti-inflammatory, antibacterial activity, antibiotic resistance, anticancer activity, 
antimicrobial activity, cytotoxicity, fungicide, insecticide, tissue engineering, and wound healing, etc. They also studied breast cancer, cancer, apoptosis, diabetes, and Alzheimer's disease. The interconnectivity between these keywords is described in Figure 6 .
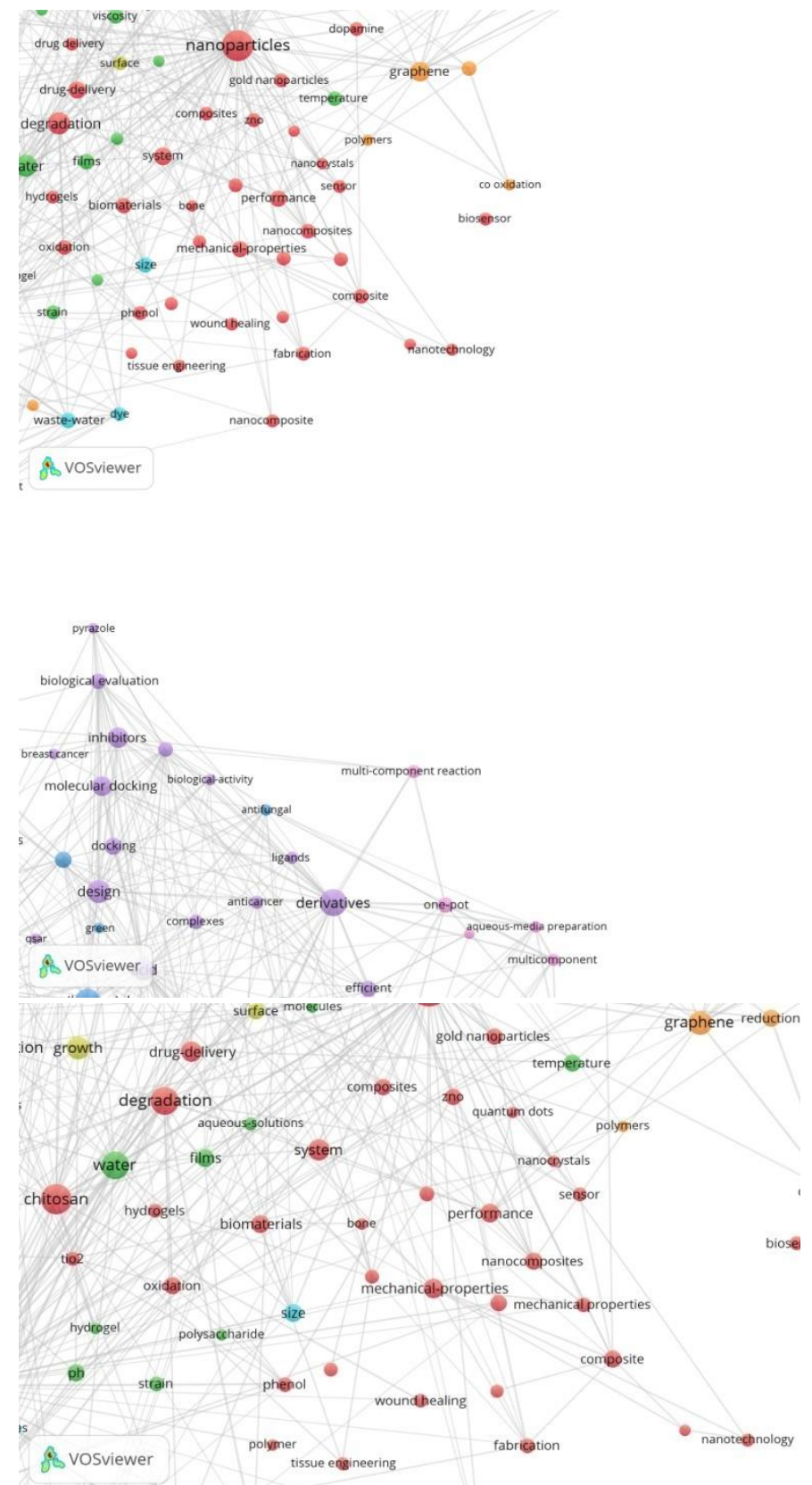
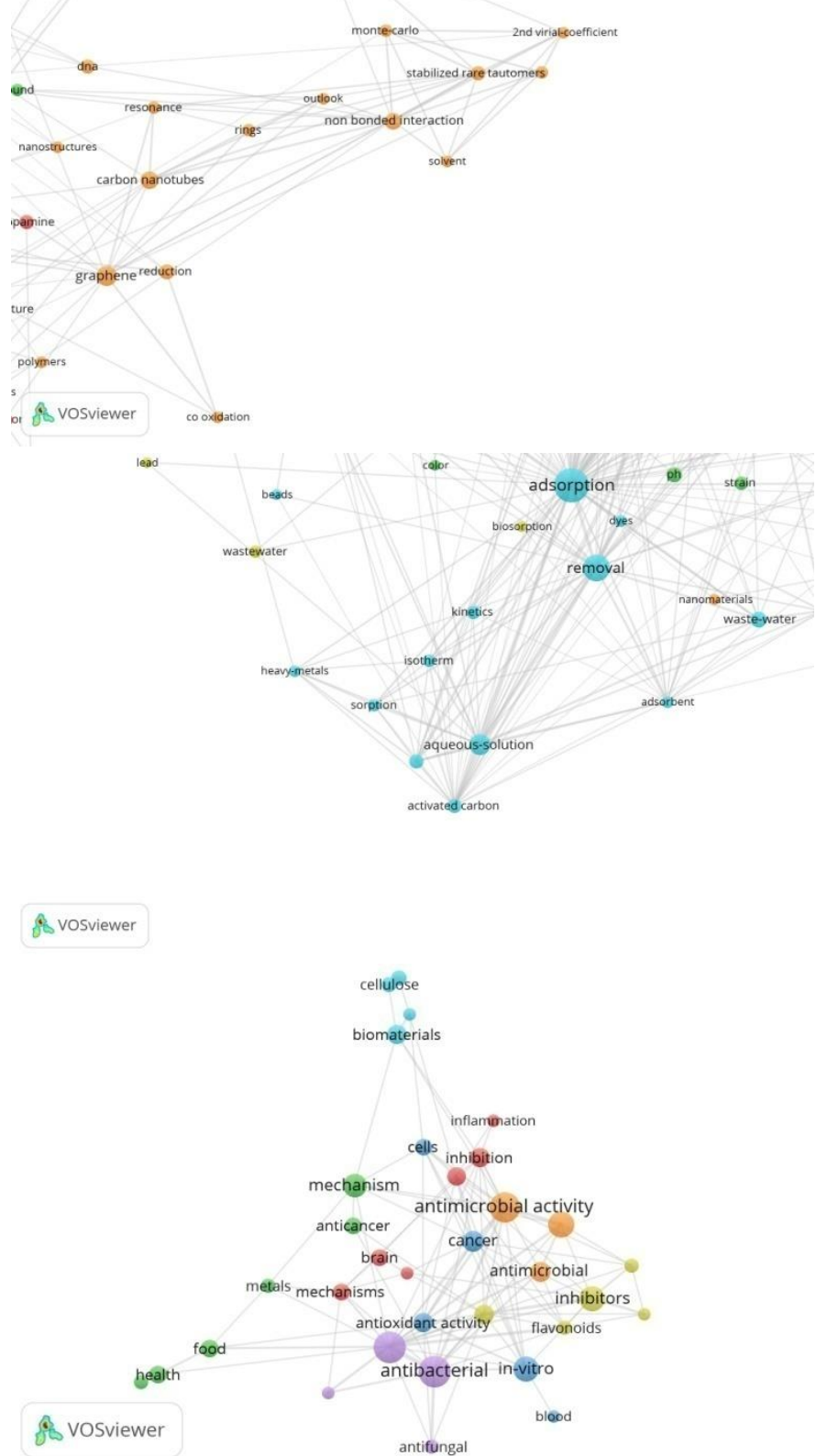

Figure 6. Co-words analysis for keywords. Different clusters are described, which represent major keywords.

\section{Conclusions}

We retrieved the citation and relevant data from Web of Science. It is worthy to note that the unofficial impact factor was found to be 1.93 (according to the Web of Science database) (498 citations in 2020 divided by 258 papers published in 2018-2019), meaning that the journal fits in the Q2 zone for applied chemistry. This shows remarkable growth in a short span of time. 


\section{Limitations}

There are some limitations to our study. We noticed writing mistakes in affiliations. For example, in one format, they described post box numbers, while in another, the authors did not add postal addresses. We only performed co-words analysis for keywords. The full-text publications were not analyzed for detailed understanding.

\section{Funding}

This research received no external funding.

\section{Acknowledgments}

Special thanks to all contributors that support the journal.

\section{Conflicts of Interest}

The authors declare no conflict of interest.

\section{References}

1. Muhuri, P.K.; Shukla, A.K.; Abraham, A. Industry 4.0: A bibliometric analysis and detailed overview. Eng. Appl. Artif. Intell 2019, 78, 218-235, https://doi.org/10.1016/j.engappai.2018.11.007.

2. Nunen, V.K.; J, Li..; Reniers. G.; \& Ponnet, K. Bibliometric analysis of safety culture research. Saf Sci 2018, 108, 248-258, https://doi.org/10.1016/j.ssci.2017.08.011.

3. Okumus, B.; Koseoglu, M.A.; \& Ma, F. Food and gastronomy research in tourism and hospitality: A bibliometric analysis. Int. J. Hosp. Manag 2018, 73, 64-74, https://doi.org/10.1016/j.ijhm.2018.01.020.

4. Grumezescu, A.M. Editorial: Celebrating ten years of platinum open access. Biointerface Res. Appl. Chem. 2020, 10, 4705, https://doi.org/10.33263/BRIAC101.705705.

5. Lin, H.; Zhu, Y.; Ahmad, N.; Han, Q. A scientometric analysis and visualization of global research on brownfields. ENVIRON SCI POLLUT R 2019, 26, 17666-17684, https://doi.org/10.1007/s11356-019-051493.

6. Bruna de Paula, FF.; Sampaio, R.B.; Fonseca, M.V.; Zicker, F. Co-authorship network analysis in health research: method and potential use. Health Res. Policy Syst 2016, 14, 34, https://doi.org/10.1186/s12961016-0104-5.

7. Bornmann, L.; Leydesdorff, L. Topical connections between the institutions within an organisation (institutional co-authorships, direct citation links and co-citations). Scientometrics 2015, 102, 455-463, https://doi.org/10.1007/s11192-014-1425-1. 


\section{Supplementary material}

Table S1. List of 2519 authors with total documents and citations. Author

\begin{tabular}{|c|c|c|}
\hline & & \\
\hline Monajjemi, Majid & 24 & 73 \\
\hline Husain, Qayyum & 4 & 62 \\
\hline Ibrahim, Medhat & 14 & 57 \\
\hline Ibrahim, Medhat A. & 8 & 57 \\
\hline Elhaes, Hanan & 15 & 55 \\
\hline Mollaamin, Fatemeh & 18 & 49 \\
\hline Khosravani, Masood & 5 & 44 \\
\hline Ghashang, Majid & 7 & 43 \\
\hline Naidu, Kadiyala Chandra Babu & 4 & 34 \\
\hline Chifiriuc, Mariana Carmen & 14 & 33 \\
\hline Adabi, Mohsen & 3 & 30 \\
\hline Esnaashari, Seyedeh Sara & 3 & 30 \\
\hline Khosravi-Darani, Kianoush & 9 & 29 \\
\hline Bassem, Samah M. & 2 & 29 \\
\hline El-Taweel, Gamila E. & 2 & 29 \\
\hline Sabry, Noha M. & 2 & 29 \\
\hline Elazab, Hany A. & 5 & 27 \\
\hline Hadibarata, Tony & 8 & 26 \\
\hline Gheorghe, Irina & 7 & 26 \\
\hline Adabi, Mahdi & 3 & 26 \\
\hline Foroughifar, Naser & 8 & 23 \\
\hline Bayoumy, Ahmed M. & 5 & 23 \\
\hline Pasdar, Hoda & 7 & 22 \\
\hline Mozafari, Masoud & 4 & 22 \\
\hline Chien Mau Dang & 8 & 21 \\
\hline Badry, Rania & 7 & 21 \\
\hline Kumar, Kaushik & 5 & 21 \\
\hline Ravinder, Dachepalli & 3 & 21 \\
\hline Yahia, Ibrahim S. & 5 & 20 \\
\hline Zahran, Heba Y. & 5 & 20 \\
\hline Lazar, Veronica & 13 & 19 \\
\hline Naghibzadeh, Majid & 2 & 19 \\
\hline Abdel-Gawad, Fagr Kh. & 1 & 19 \\
\hline Nassar, Hossam F. & 1 & 19 \\
\hline Okasha, Aly & 1 & 19 \\
\hline Saffar-Teluri, Ali & 1 & 19 \\
\hline Tolba, Sahar & 1 & 19 \\
\hline Zare, Mohsen & 1 & 19 \\
\hline Dung My Thi Dang & 7 & 18 \\
\hline Chaban, Taras & 5 & 18 \\
\hline Matiychuk, Vasyl & 5 & 18 \\
\hline Adabi, Mandi & 2 & 18 \\
\hline Valizadeh, Alireza & 2 & 18 \\
\hline Mandal, Badal Kumar & 6 & 17 \\
\hline Refaat, Ahmed & 5 & 17 \\
\hline Thu Thi Pham & 5 & 17 \\
\hline Khamehchi, Ehsan & 3 & 16 \\
\hline Faisal, Nadeem & 2 & 16 \\
\hline Rezvani, Zahra & 2 & 16 \\
\hline Abdolghaffari, Amir Hossein & 1 & 16 \\
\hline Abdollahi, Mohammad & 1 & 16 \\
\hline Amanzadi, Bentolhoda & 1 & 16 \\
\hline Boroumand, Safieh & 1 & 16 \\
\hline Hassanzadeh, Gholamreza & 1 & 16 \\
\hline Majidi, Reza Faridi & 1 & 16 \\
\hline Mandaviani, Parvin & 1 & 16 \\
\hline Mirzaei, Esmaeil & 1 & 16 \\
\hline
\end{tabular}




\begin{tabular}{|c|c|c|}
\hline Author & Documents & Citations \\
\hline Fahmy, Ahmed & 3 & 15 \\
\hline Ali, Imran & 1 & 15 \\
\hline Hsieh, Ming-Fa & 1 & 15 \\
\hline Lone, Mohammad Nadeem & 1 & 15 \\
\hline Syafiuddin, Achmad & 9 & 14 \\
\hline Franca, Eduardo Luzia & 3 & 14 \\
\hline Osman, Osama & 3 & 14 \\
\hline Antonia Brandan, Silvia & 5 & 13 \\
\hline Ezzat, Hend & 4 & 13 \\
\hline Dias-Souza, Marcus Vinicius & 2 & 13 \\
\hline Honorio-Franca, Adenilda Cristina & 2 & 13 \\
\hline Hussein, Tarek & 2 & 13 \\
\hline Leite, Fabio De Lima & 2 & 13 \\
\hline Mihaescu, Grigore & 2 & 13 \\
\hline Anuchina, N. M. & 1 & 13 \\
\hline Krayukhina, M. A. & 1 & 13 \\
\hline Kumar, Rapole Jeevan & 1 & 13 \\
\hline Naresh, U. & 1 & 13 \\
\hline Piskarev, V. E. & 1 & 13 \\
\hline Popov, D. A. & 1 & 13 \\
\hline Samoilova, N. A. & 1 & 13 \\
\hline Shafiee, Mohammad Reza Mohammad & 4 & 12 \\
\hline Omar, Amina & 3 & 12 \\
\hline Abdel-Fattah, Wafa, I & 2 & 12 \\
\hline Basha, D. Baba & 1 & 12 \\
\hline Kumar, Nagasamudram Suresh & 1 & 12 \\
\hline Ramesh, Singampalli & 1 & 12 \\
\hline Sekhar, B. Chandra & 1 & 12 \\
\hline Srinivas, Kurapati & 1 & 12 \\
\hline Al-Ghamdi, Abdullah Ahmed & 3 & 11 \\
\hline Bayraktar, Oguz & 3 & 11 \\
\hline Cristea, Violeta Corina & 3 & 11 \\
\hline Movafeghi, Ali & 3 & 11 \\
\hline Akil, Hazizan Md & 2 & 11 \\
\hline Jamila, Nargis & 2 & 11 \\
\hline Javed, Fatima & 2 & 11 \\
\hline Khan, Abdul Naeem & 2 & 11 \\
\hline Shahriari, Sara & 2 & 11 \\
\hline Ullah, Faheem & 2 & 11 \\
\hline Ayodele, Adebileje Tajudeen & 1 & 11 \\
\hline Batra, Navneet & 1 & 11 \\
\hline Goel, Abhishek & 1 & 11 \\
\hline Madani, Fatemeh & 1 & 11 \\
\hline Phutela, Kanika & 1 & 11 \\
\hline Pricop, Roxana & 1 & 11 \\
\hline Sharma, Neetu & 1 & 11 \\
\hline Soni, Sajeev & 1 & 11 \\
\hline Tatu, Alin Laurentiu & 1 & 11 \\
\hline Denizli, Adil & 5 & 10 \\
\hline Farkhondeh, Tahereh & 5 & 10 \\
\hline Samarghandian, Saeed & 5 & 10 \\
\hline Endarko, Endarko & 3 & 10 \\
\hline Mortazavian, Amir M. & 3 & 10 \\
\hline Popa, Marcela & 3 & 10 \\
\hline Barakat, Khouloud M. & 2 & 10 \\
\hline El-Idreesy, Tamer T. & 2 & 10 \\
\hline Jiang, Jia-Qian & 2 & 10 \\
\hline Ogurtsov, Volodymyr & 2 & 10 \\
\hline Abdel-Gawad, Fagr Kh & 1 & 10 \\
\hline Abdelghany, Amr Mohamed & 1 & 10 \\
\hline Adel, Moein & 1 & 10 \\
\hline
\end{tabular}




\begin{tabular}{|c|c|c|}
\hline Author & Documents & Citations \\
\hline Bosch, Paula & 1 & 10 \\
\hline Chauhan, Narendra Pal Singh & 1 & 10 \\
\hline El-Bana, Afaf Adel & 1 & 10 \\
\hline Fesahat, Farzaneh & 1 & 10 \\
\hline Firoozi, Saman & 1 & 10 \\
\hline Grabchev, Ivo & 1 & 10 \\
\hline Grozdanov, Peter & 1 & 10 \\
\hline Jalali, Newsha & 1 & 10 \\
\hline Khoradmehr, Arezoo & 1 & 10 \\
\hline Medel, Sandra & 1 & 10 \\
\hline Meikhail, Mahrous Shaker & 1 & 10 \\
\hline Nassar, Hossam & 1 & 10 \\
\hline Nikolova, Ivanka & 1 & 10 \\
\hline Nodoushan, Fatemeh Sadeghian & 1 & 10 \\
\hline Pazoki-Toroudi, Hamidreza & 1 & 10 \\
\hline Staneva, Desislava & 1 & 10 \\
\hline Tavakol, Shima & 1 & 10 \\
\hline Tolba, Sahar T. M. & 1 & 10 \\
\hline Vasileva-Tonkova, Evgenia & 1 & 10 \\
\hline Zahmatkeshan, Masoumeh & 1 & 10 \\
\hline Bolocan, Alexandra & 4 & 9 \\
\hline Darwesh, Osama M. & 4 & 9 \\
\hline Sharifi, Simin & 4 & 9 \\
\hline Atta, Diaa & 3 & 9 \\
\hline Bennet, Kevin E. & 3 & 9 \\
\hline Ibrahim, Asmaa & 3 & 9 \\
\hline Joice, G. Helen Ruth & 3 & 9 \\
\hline Manciu, Felicia S. & 3 & 9 \\
\hline Naik, J. Laxman & 3 & 9 \\
\hline Steffens, Juliana & 3 & 9 \\
\hline Banu, Otilia & 2 & 9 \\
\hline Ciubuc, John D. & 2 & 9 \\
\hline Eastman, Michael & 2 & 9 \\
\hline Ezzat, Hend A. & 2 & 9 \\
\hline Kargar, Mahboubeh & 2 & 9 \\
\hline Steffens, Clarice & 2 & 9 \\
\hline Abd El-Moez, Sh, I & 1 & 9 \\
\hline Ali, Ghareib W. & 1 & 9 \\
\hline Bahramian, Ghazaleh & 1 & 9 \\
\hline Dhole, Soumita & 1 & 9 \\
\hline Ghayoor, Hamid & 1 & 9 \\
\hline Golestan, Leila & 1 & 9 \\
\hline Hegazy, Maroof A. & 1 & 9 \\
\hline Khan, Fazlur-Rahman Nawaz & 1 & 9 \\
\hline Khosravian, Peyman & 1 & 9 \\
\hline Ling, Yong-Chien & 1 & 9 \\
\hline Malekpour, Mohammad Reza & 1 & 9 \\
\hline Mujokoro, Basil & 1 & 9 \\
\hline Mukhopadhyay, Oeindrila & 1 & 9 \\
\hline Nada, Nadra A. & 1 & 9 \\
\hline Najafabadi, Mohammad Reza H. & 1 & 9 \\
\hline Naidu, K. Chandra Babu & 6 & 8 \\
\hline Shah, Munir H. & 6 & 8 \\
\hline Chaib, Messaoud & 3 & 8 \\
\hline Kumar, Vinod & 3 & 8 \\
\hline Marutescu, Luminita & 3 & 8 \\
\hline Abbasi, Arshad Mehmood & 2 & 8 \\
\hline Chaban, Ihor & 2 & 8 \\
\hline Drapak, Iryna & 2 & 8 \\
\hline Hassabo, Ahmed G. & 2 & 8 \\
\hline Hassan, Sahar H. & 2 & 8 \\
\hline
\end{tabular}




\begin{tabular}{|c|c|c|}
\hline Author & Documents & Citations \\
\hline Kushkevych, Ivan & 2 & 8 \\
\hline Lelyukh, Maryan & 2 & 8 \\
\hline Mohamed, Amina L. & 2 & 8 \\
\hline Sabae, Shawky Z. & 2 & 8 \\
\hline Tozetti, Ines Aparecida & 2 & 8 \\
\hline Yilmaz, Fatma & 2 & 8 \\
\hline Adriaens, Annemie & 1 & 8 \\
\hline Akramzadeh, Naimeh & 1 & 8 \\
\hline Amoabediny, Ghassem & 1 & 8 \\
\hline Arzani, Hossein & 1 & 8 \\
\hline Brezolin, Alexandra Nava & 1 & 8 \\
\hline De Cezaro, Alana Marie & 1 & 8 \\
\hline De Keersmaecker, Michel & 1 & 8 \\
\hline Dias, Cleiton Goncalves & 1 & 8 \\
\hline Dorkoosh, Farid & 1 & 8 \\
\hline Ferreira-Marcal, Pedro Henrique & 1 & 8 \\
\hline Gusev, Aleksandr I. & 1 & 8 \\
\hline Hosseini, Morteza & 1 & 8 \\
\hline Kamali, Morteza & 1 & 8 \\
\hline Khashayar, Patricia & 1 & 8 \\
\hline Koller, Martin & 1 & 8 \\
\hline Larijani, Bagher & 1 & 8 \\
\hline Maleki, Hasan & 1 & 8 \\
\hline Manzoli, Alexandra & 1 & 8 \\
\hline Martinazzo, Janine & 1 & 8 \\
\hline Mosafer, Jafar & 1 & 8 \\
\hline Muenchen, Daniela Kunkel & 1 & 8 \\
\hline Nekounam, Houra & 1 & 8 \\
\hline Rigo, Aline Andressa & 1 & 8 \\
\hline Sadovnikov, Stanislav I. & 1 & 8 \\
\hline Schaubroeck, David & 1 & 8 \\
\hline Vanfleteren, Jan & 1 & 8 \\
\hline Verplancke, Rik & 1 & 8 \\
\hline Tebyanian, Hamid & 6 & 7 \\
\hline Lashkari, Mojtaba & 5 & 7 \\
\hline Dizaj, Solmaz Maleki & 4 & 7 \\
\hline Nugrahani, Ilma & 4 & 7 \\
\hline Tociu, Carmen & 4 & 7 \\
\hline De Melo Cotrim, Aron Carlos & 2 & 7 \\
\hline Divband, Baharak & 2 & 7 \\
\hline Fatima, Nighat & 2 & 7 \\
\hline Jaiswal, Krishna Kumar & 2 & 7 \\
\hline Zare, Karim & 2 & 7 \\
\hline Amini, Elahe & 1 & 7 \\
\hline Arratia-Perez, Ramiro & 1 & 7 \\
\hline Azadfallah, Mohammad & 1 & 7 \\
\hline Bienek, Diane R. & 1 & 7 \\
\hline Brainina, Khiena Z. & 1 & 7 \\
\hline Chandia, Nancy & 1 & 7 \\
\hline Da Silva Fernandes, Rubian Trindade & 1 & 7 \\
\hline De Oliveira Silva, Bruna Santiago & 1 & 7 \\
\hline Durrer, William G. & 1 & 7 \\
\hline El-Khodary, Sherif A. & 1 & 7 \\
\hline El-Sayed, El-Sayed M. & 1 & 7 \\
\hline Feres Machi, Patricia Gelli & 1 & 7 \\
\hline Fujimori, Mahmi & 1 & 7 \\
\hline Geraldo, Daniela A. & 1 & 7 \\
\hline Gomes Fagundes Triches, Danny Laura & 1 & 7 \\
\hline Goswami, Anuradha & 1 & 7 \\
\hline Hara, Seth & 1 & 7 \\
\hline Karimi, Fatemeh & 1 & 7 \\
\hline
\end{tabular}




\begin{tabular}{|c|c|c|}
\hline Author & Documents & Citations \\
\hline Kazakov, Yan E. & 1 & 7 \\
\hline Kazemi, Elham Mohajel & 1 & 7 \\
\hline Keshvadi, Mohammadhosein & 1 & 7 \\
\hline Kholmurodov, Kholmirzo T. & 1 & 7 \\
\hline Kosari-Nasab, Morteza & 1 & 7 \\
\hline Kotrsova, Vera & 1 & 7 \\
\hline Mahjouri, Sepideh & 1 & 7 \\
\hline Massmman, Patricia Fernandes & 1 & 7 \\
\hline Mattar, Magdy Z. & 1 & 7 \\
\hline Mcintosh, Malcolm B. & 1 & 7 \\
\hline Mora, Guido C. & 1 & 7 \\
\hline Morsy, Mohamed & 1 & 7 \\
\hline Needham, Paula & 1 & 7 \\
\hline Qiu, Chao & 1 & 7 \\
\hline Schmidt, Ute & 1 & 7 \\
\hline Seabra, Amedea Barozzi & 1 & 7 \\
\hline Skrtic, Drago & 1 & 7 \\
\hline Tomshine, Jonathan R. & 1 & 7 \\
\hline Valizadeh, Siavash & 1 & 7 \\
\hline Varzakova, Daria P. & 1 & 7 \\
\hline Vidrevich, Marina B. & 1 & 7 \\
\hline Villagra, Nicolas A. & 1 & 7 \\
\hline Zaid, Hamdya & 1 & 7 \\
\hline Baltaci, Abdulkerim Kasim & 5 & 6 \\
\hline Mogulkoc, Rasim & 5 & 6 \\
\hline Hazeri, Nourallah & 4 & 6 \\
\hline Anghel, Ana-Maria & 3 & 6 \\
\hline Chanh Minh Thi Le & 3 & 6 \\
\hline Hosseini, Hedayat & 3 & 6 \\
\hline Ilie, Mihaela & 3 & 6 \\
\hline Marinescu, Florica & 3 & 6 \\
\hline Naskar, Atanu & 3 & 6 \\
\hline Rao, B. Ramakrishna & 3 & 6 \\
\hline Utrera Martines, Marco Antonio & 3 & 6 \\
\hline Ahmed, Kawsar & 2 & 6 \\
\hline Ashrafizadeh, Milad & 2 & 6 \\
\hline Barkhordari, Aref & 2 & 6 \\
\hline Baskaran, Iruson & 2 & 6 \\
\hline Bhuyian, Touhid & 2 & 6 \\
\hline Dang, Chien Mau & 2 & 6 \\
\hline Davallo, Mehran & 2 & 6 \\
\hline Hashim, Mohd & 2 & 6 \\
\hline Jana, Sunirmal & 2 & 6 \\
\hline Karami, Ali & 2 & 6 \\
\hline Killivalavan, Govindarasu & 2 & 6 \\
\hline Manikandan, Elayaperumal & 2 & 6 \\
\hline Naghsh, Farnoush & 2 & 6 \\
\hline Paul, Bikash Kumar & 2 & 6 \\
\hline Prabakar, Arthur Charles & 2 & 6 \\
\hline Rasoolzadeh, Reza & 2 & 6 \\
\hline Sathyaseelan, Balaraman & 2 & 6 \\
\hline Senthilnathan, Krishnamoorthy & 2 & 6 \\
\hline Sivakumar, Dhananjayan & 2 & 6 \\
\hline Soufdoost, Reza Sayyad & 2 & 6 \\
\hline Zindani, Divya & 2 & 6 \\
\hline Adeyanju, Anne Adebukola & 1 & 6 \\
\hline Adeyemi, Oluyomi Stephen & 1 & 6 \\
\hline Alamri, Saud A. & 1 & 6 \\
\hline Awakan, Oluwakemi Josephine & 1 & 6 \\
\hline Beresteanu, Serban Vifor & 1 & 6 \\
\hline Caponetti, Eugenio & 1 & 6 \\
\hline
\end{tabular}




\begin{tabular}{|c|c|c|}
\hline Author & Documents & Citations \\
\hline Chakravarthula, Sarada Nallani & 1 & 6 \\
\hline Colomba, Paola & 1 & 6 \\
\hline Demirbag, Caglar & 1 & 6 \\
\hline Ezzati, Shoaib & 1 & 6 \\
\hline Farhadihosseinabadi, Behrouz & 1 & 6 \\
\hline Faridi-Majidi, Reza & 1 & 6 \\
\hline Gholipourmalekabadi, Mazaher & 1 & 6 \\
\hline Girasolo, Maria Assunta & 1 & 6 \\
\hline Grigore, Raluca & 1 & 6 \\
\hline Hidayat, Topik & 1 & 6 \\
\hline Kanthasamy, Shivashanggeeta & 1 & 6 \\
\hline Khafagy, Rasha M. & 1 & 6 \\
\hline Kudus, Muhammad Helmi Abdul & 1 & 6 \\
\hline Martino, Delia F. Chillura & 1 & 6 \\
\hline Merezeanu, Nicoleta & 1 & 6 \\
\hline Minhaz, Aaliya & 1 & 6 \\
\hline Mosaddad, Seyed Ali & 1 & 6 \\
\hline Najafi, Parisa Zarif & 1 & 6 \\
\hline Narasaiah, Boya Palajonna & 1 & 6 \\
\hline Niri, Alireza Dehghan & 1 & 6 \\
\hline Olajide, Isaac Oluwafemi & 1 & 6 \\
\hline Otohinoyi, David Adeiza & 1 & 6 \\
\hline Pantea, Octav & 1 & 6 \\
\hline Peivasteh-Roudsari, Leila & 1 & 6 \\
\hline Perchyonok, Victoria Tamara & 1 & 6 \\
\hline Rubino, Simona & 1 & 6 \\
\hline Saber, Reza & 1 & 6 \\
\hline Saladino, Maria Luisa & 1 & 6 \\
\hline Salari, Yalda & 1 & 6 \\
\hline Samadikuchaksaraei, Ali & 1 & 6 \\
\hline Tahmasebi, Elaheh & 1 & 6 \\
\hline Yazdanian, Alireza & 1 & 6 \\
\hline Yazdanian, Mohsen & 1 & 6 \\
\hline Mohamadpour, Farzaneh & 4 & 5 \\
\hline Abdelghany, Amr M. & 3 & 5 \\
\hline Csutak, Ortansa & 3 & 5 \\
\hline Maghsoodlou, Malek Taher & 3 & 5 \\
\hline Alaraidh, Ibrahim A. & 2 & 5 \\
\hline Ali, Salah Fawzi Abdellah & 2 & 5 \\
\hline Alsahli, Abdulaziz A. & 2 & 5 \\
\hline Badaoui, Mohamed & 2 & 5 \\
\hline Dehbalaei, Masoumeh Gholami & 2 & 5 \\
\hline Dewi, Ratni & 2 & 5 \\
\hline Elwina, Elwina & 2 & 5 \\
\hline Gaur, Praveen Kumar & 2 & 5 \\
\hline Matiichuk, Yuliia & 2 & 5 \\
\hline Mokhtarpour, Masumeh & 2 & 5 \\
\hline Omrani, Maryam Mehdizadeh & 2 & 5 \\
\hline Ostapiuk, Yuriy & 2 & 5 \\
\hline Puri, Dinesh & 2 & 5 \\
\hline Ratnasari, Anisa & 2 & 5 \\
\hline Romani, Davide & 2 & 5 \\
\hline Shanmugam, Sadish Kumar & 2 & 5 \\
\hline Srivastava, Abhishek & 2 & 5 \\
\hline Zarshenas, Mohammad M. & 2 & 5 \\
\hline Ziane, Belkacem & 2 & 5 \\
\hline Abdelrazek, Elmetwally Mohamed M. & 1 & 5 \\
\hline Al Qahtani, Hamad M. S. & 1 & 5 \\
\hline Alotaibi, Mohammed & 1 & 5 \\
\hline Amer, Hamada & 1 & 5 \\
\hline Ansari, Mojtaba & 1 & 5 \\
\hline
\end{tabular}




\begin{tabular}{|c|c|c|}
\hline Author & Documents & Citations \\
\hline Bahri, Afshin & 1 & 5 \\
\hline Barbosa Figueiredo, Flavio Junior & 1 & 5 \\
\hline Bera, Susanta & 1 & 5 \\
\hline Bhattacharya, Rahul & 1 & 5 \\
\hline Carmen, Mariana Chifiriuc & 1 & 5 \\
\hline Ceravolo, Isabela Penna & 1 & 5 \\
\hline Corina, Cristea Violeta & 1 & 5 \\
\hline Ejtemaee, Pedram & 1 & 5 \\
\hline Fajar, Muhammad Noer & 1 & 5 \\
\hline Farrage, Neveen Mohamed & 1 & 5 \\
\hline Feldman, Dorel & 1 & 5 \\
\hline Fojan, Peter & 1 & 5 \\
\hline Foliush, Volodymyr & 1 & 5 \\
\hline Hanif, Muhammad & 1 & 5 \\
\hline Hausen, Moema & 1 & 5 \\
\hline Higa, Akemi Martins & 1 & 5 \\
\hline Irina, Gheorghe & 1 & 5 \\
\hline Jimena Marquez, Maria & 1 & 5 \\
\hline Juluri, Raghavendra R. & 1 & 5 \\
\hline Khalid, Mohamed & 1 & 5 \\
\hline Khattak, Rozina & 1 & 5 \\
\hline Kiaie, Nasim & 1 & 5 \\
\hline Kose, Merve Deniz & 1 & 5 \\
\hline Kristanti, Risky Ayu & 1 & 5 \\
\hline Mahlovanyy, Anatoliy & 1 & 5 \\
\hline Malek, Nik Ahmad Nizam Nik & 1 & 5 \\
\hline Mambrini, Giovanni Pimenta & 1 & 5 \\
\hline Marcela, Popa & 1 & 5 \\
\hline Moein, Mahmoodreza & 1 & 5 \\
\hline Nicoleta, Merezeanu & 1 & 5 \\
\hline Octav, Pantea & 1 & 5 \\
\hline Oraby, Ahmed Hamza & 1 & 5 \\
\hline Otilia, Banu & 1 & 5 \\
\hline Popok, Vladimir N. & 1 & 5 \\
\hline Radwan, Ahmed & 1 & 5 \\
\hline Rameshkumar, Gubendiran & 1 & 5 \\
\hline Rawat, Bhupendra Singh & 1 & 5 \\
\hline Rodrigues Dos Santos, Lucas David & 1 & 5 \\
\hline Roy, Sib Sankar & 1 & 5 \\
\hline Rubiyanto, Agus & 1 & 5 \\
\hline Saghavaz, Bahare Hedyati & 1 & 5 \\
\hline Salehi, Roghayeh & 1 & 5 \\
\hline Shojaei, Shahrokh & 1 & 5 \\
\hline Soares Dos Santos, Agatha Emanuelle & 1 & 5 \\
\hline Strixino, Francisco Trivinho & 1 & 5 \\
\hline Talebi, Marjan & 1 & 5 \\
\hline Tatu, Laurentiu Alin & 1 & 5 \\
\hline Veronica, Lazar & 1 & 5 \\
\hline Yazdani, Elahe & 1 & 5 \\
\hline Zakaria, Muhammad Razlan & 1 & 5 \\
\hline Khajeh-Amiri, Alireza & 4 & 4 \\
\hline Pignatello, Rosario & 4 & 4 \\
\hline Samrot, Antony, V & 4 & 4 \\
\hline Vassu, Tatiana & 4 & 4 \\
\hline Al-Anber, Mohammed A. & 3 & 4 \\
\hline Bozdogan, Adnan & 3 & 4 \\
\hline Dallago, Rogerio Marcos & 3 & 4 \\
\hline Delcaru, Cristina & 3 & 4 \\
\hline Ermolaev, Vladimir Alexandrovich & 3 & 4 \\
\hline Fuochi, Virginia & 3 & 4 \\
\hline Furneri, Pio Maria & 3 & 4 \\
\hline
\end{tabular}




\begin{tabular}{|c|c|c|}
\hline Author & Documents & Citations \\
\hline Hamzah, Sofiah & 3 & 4 \\
\hline Khan, Ikramullah & 3 & 4 \\
\hline Podgoreanu, Paulina & 3 & 4 \\
\hline Shuaib, Muhammad & 3 & 4 \\
\hline Ulukanli, Zeynep & 3 & 4 \\
\hline Ahangari, Ghasem & 2 & 4 \\
\hline De Castro, Gustavo Rocha & 2 & 4 \\
\hline Demaman Oro, Carolina Elisa & 2 & 4 \\
\hline Guastaldi, Antonio C. & 2 & 4 \\
\hline Kameshwar, Vivek Hamse & 2 & 4 \\
\hline Kariyappa, Ajay Kumar & 2 & 4 \\
\hline Keshvari, Hamid & 2 & 4 \\
\hline Meng, Fanli & 2 & 4 \\
\hline Mohammed, Salam A. & 2 & 4 \\
\hline Narendrakumar, G. & 2 & 4 \\
\hline Petronio, Giulio Petronio & 2 & 4 \\
\hline Ponnaiah, Paulraj & 2 & 4 \\
\hline Rodriguez-Chanfrau, Jorge E. & 2 & 4 \\
\hline Salati, Amir & 2 & 4 \\
\hline Sanati, Mohammad Hossein & 2 & 4 \\
\hline Vahed, Sepideh Zununi & 2 & 4 \\
\hline Yousif, Emad & 2 & 4 \\
\hline Achutha, Dileep Kumar & 1 & 4 \\
\hline Afradi, Nahid & 1 & 4 \\
\hline Afrin, Shaik & 1 & 4 \\
\hline Ahmed, Minhaz Uddin & 1 & 4 \\
\hline Aliah, Ahmad Irsyad & 1 & 4 \\
\hline Alyoshina, Lyudmila & 1 & 4 \\
\hline Amor, Ali & 1 & 4 \\
\hline Anuar, Fatin Izzaidah & 1 & 4 \\
\hline Asir, Suleyman & 1 & 4 \\
\hline Babu, Puttagunta Srinivasa & 1 & 4 \\
\hline Baysal, Zubeyde & 1 & 4 \\
\hline Benali, Mouffok & 1 & 4 \\
\hline Benincasa, Giulio & 1 & 4 \\
\hline Bibi, Nazma & 1 & 4 \\
\hline Brainina, Khiena & 1 & 4 \\
\hline Buia, Ruxandra & 1 & 4 \\
\hline Cakir, Oguz & 1 & 4 \\
\hline Chawla, Surinder Pritamdas & 1 & 4 \\
\hline Crisci, Alessandro & 1 & 4 \\
\hline Crisci, Francesco & 1 & 4 \\
\hline Crisci, Michela & 1 & 4 \\
\hline Derazshamshir, Ali & 1 & 4 \\
\hline Duman, Aysel & 1 & 4 \\
\hline Fadlilah, Dian Rosyida & 1 & 4 \\
\hline Fona, Zahra & 1 & 4 \\
\hline Gokturk, Ilgim & 1 & 4 \\
\hline Greco, Antonio S. & 1 & 4 \\
\hline Haq, Sirajul & 1 & 4 \\
\hline Harish, Bachala Sai & 1 & 4 \\
\hline Hatefi, Yeganeh & 1 & 4 \\
\hline Hozairi, Hozairi & 1 & 4 \\
\hline Jayalakshmi, Basavegowda & 1 & 4 \\
\hline Kanatt, Sweetie Raju & 1 & 4 \\
\hline Karamad, Dina & 1 & 4 \\
\hline Kazakov, Yan & 1 & 4 \\
\hline Keasberry, Natasha Ann & 1 & 4 \\
\hline Khadivi, Ramona & 1 & 4 \\
\hline Khan, Basharat & 1 & 4 \\
\hline Khane, Yasmine & 1 & 4 \\
\hline
\end{tabular}




\begin{tabular}{|c|c|c|}
\hline Author & Documents & Citations \\
\hline Kumar, Basappa Chidanda Vasantha & 1 & 4 \\
\hline Kumar, Nitin & 1 & 4 \\
\hline Lahcene, Belarbi & 1 & 4 \\
\hline Lopez-Malo, Aurelio & 1 & 4 \\
\hline Mejia-Garibay, Beatriz & 1 & 4 \\
\hline Mohamad, Azureen & 1 & 4 \\
\hline Navarro-Amador, Ricardo & 1 & 4 \\
\hline Negrea, Stefania Madalina & 1 & 4 \\
\hline Ningappa, Mylarappa Bantaganahalli & 1 & 4 \\
\hline Pal, Himanshu & 1 & 4 \\
\hline Palou, Enrique & 1 & 4 \\
\hline Palu, Serge & 1 & 4 \\
\hline Pioch, Daniel & 1 & 4 \\
\hline Punvichai, Teerasak & 1 & 4 \\
\hline Qomi, Mahnaz & 1 & 4 \\
\hline Ramedani, Arash & 1 & 4 \\
\hline Ramirez-Corona, Nelly & 1 & 4 \\
\hline Rasheed, Parsa & 1 & 4 \\
\hline Rehman, Mahfooz Ur & 1 & 4 \\
\hline Rehman, Wajid & 1 & 4 \\
\hline Rekha, Nangappagowda Dharmappa & 1 & 4 \\
\hline $\begin{array}{l}\text { Revanasiddappa, Hosakere Doddarevanna } \\
\text { L. }\end{array}$ & 1 & 4 \\
\hline Rizwan, Mohammad & 1 & 4 \\
\hline Sari, Duygu & 1 & 4 \\
\hline Shaban, Hassanein & 1 & 4 \\
\hline Shah, Amreen & 1 & 4 \\
\hline Shaik, Afzal Basha & 1 & 4 \\
\hline Sharma, Disha & 1 & 4 \\
\hline Siddiqui, Anam & 1 & 4 \\
\hline Sivrikaya, Abdullah & 1 & 4 \\
\hline Soto-Chilaca, Gerardo A. & 1 & 4 \\
\hline Supraja, Kondapalli & 1 & 4 \\
\hline Syafrudin, Muhammad & 1 & 4 \\
\hline Tarasov, Aleksey & 1 & 4 \\
\hline Tardan, Eric & 1 & 4 \\
\hline Tavasoli, Sanaz & 1 & 4 \\
\hline Trusca, Simona Bianca & 1 & 4 \\
\hline Vani, Katti Lohitha Satya & 1 & 4 \\
\hline Waseem, Muhammad & 1 & 4 \\
\hline Yusop, Zulkifli & 1 & 4 \\
\hline Lauro, Figueroa-Valverde & 16 & 3 \\
\hline Marcela, Rosas-Nexticapa & 16 & 3 \\
\hline Maria, Lopez-Ramos & 16 & 3 \\
\hline Virginia, Mateu-Armand & 9 & 3 \\
\hline Barak, Sheweta & 5 & 3 \\
\hline Curutiu, Carmen & 5 & 3 \\
\hline Marcu, Ecaterina & 5 & 3 \\
\hline Mudgil, Deepak & 5 & 3 \\
\hline Avdeenko, Anatoly & 4 & 3 \\
\hline Ningaiah, Srikantamurthy & 4 & 3 \\
\hline Ratnamala, A. & 4 & 3 \\
\hline Sohrabvandi, Sara & 4 & 3 \\
\hline Basavanna, Vrushabendra & 3 & 3 \\
\hline Lingegowda, Nagarakere S. & 3 & 3 \\
\hline Vassu-Dimov, Tatiana & 3 & 3 \\
\hline Adrom, Belgheis & 2 & 3 \\
\hline Ahmed, Eman F. & 2 & 3 \\
\hline Dewanto, Gana Lahirin & 2 & 3 \\
\hline Doddamani, Shridevi & 2 & 3 \\
\hline Haroun, Ahmed A. & 2 & 3 \\
\hline
\end{tabular}




\begin{tabular}{|c|c|c|}
\hline Author & Documents & Citations \\
\hline Ionescu, Robertina & 2 & 3 \\
\hline Jahangiri, Azin & 2 & 3 \\
\hline Kafil, Hossein Samadi & 2 & 3 \\
\hline Kirboga, Semra & 2 & 3 \\
\hline Kumar, Sanjay & 2 & 3 \\
\hline Muruganandham, Chandramohan & 2 & 3 \\
\hline Oner, Mualla & 2 & 3 \\
\hline Pratama, Mohammad Rizki Fadhil & 2 & 3 \\
\hline Sadek, Mohamed A. & 2 & 3 \\
\hline Sarbu, Ionela & 2 & 3 \\
\hline Tambunan, Usman Sumo Friend & 2 & 3 \\
\hline Umesha, Kumbaradoddi B. & 2 & 3 \\
\hline Veranes-Pantoja, Yaymarilis & 2 & 3 \\
\hline Abdullah, Wan Rafizah Wan & 1 & 3 \\
\hline Ahmad, Rafiq & 1 & 3 \\
\hline Ahmadian, Elham & 1 & 3 \\
\hline Ahmed, Hanan M. & 1 & 3 \\
\hline Ahmed, Md Liton & 1 & 3 \\
\hline Akbarzadeh, Abolfazl & 1 & 3 \\
\hline Al-Anber, Mohammed Ahmed & 1 & 3 \\
\hline Al-Anber, Zaid Ahmed & 1 & 3 \\
\hline Ali, Asmadi & 1 & 3 \\
\hline Ali, Gharieb W. & 1 & 3 \\
\hline Almogbel, Mohammed Suliman & 1 & 3 \\
\hline Altiok, Evren & 1 & 3 \\
\hline Amara, Venkateswara Rao & 1 & 3 \\
\hline Amer, Mohamed S. & 1 & 3 \\
\hline Antunes, Angela & 1 & 3 \\
\hline Anumakonda, Varadarajulu & 1 & 3 \\
\hline Asma, Dilek & 1 & 3 \\
\hline Atalay, Funda Ersoy & 1 & 3 \\
\hline Ayat, Moulkheir & 1 & 3 \\
\hline Azaman, Fazureen & 1 & 3 \\
\hline Azami, Zahra Khalili & 1 & 3 \\
\hline Bakshi, Vasudha & 1 & 3 \\
\hline Basmaji, Pierre & 1 & 3 \\
\hline Batoo, Khalid Mujasam & 1 & 3 \\
\hline Belov, Alexey Alexeevich & 1 & 3 \\
\hline Beyramabadi, S. Ali & 1 & 3 \\
\hline Bilal, Muhammad & 1 & 3 \\
\hline Bingol, Alper & 1 & 3 \\
\hline Boda, Nehru & 1 & 3 \\
\hline Bozok, Fuat & 1 & 3 \\
\hline Buyukalaca, Saadet & 1 & 3 \\
\hline Buyukoz, Melda Y. & 1 & 3 \\
\hline Chigondo, Fidelis & 1 & 3 \\
\hline Demchuk, Inna & 1 & 3 \\
\hline Derakhshankhah, Hossein & 1 & 3 \\
\hline Dogan, Hasan Huseyin & 1 & 3 \\
\hline Dosadina, Elina Eldarovna & 1 & 3 \\
\hline Dubuis, Pascal & 1 & 3 \\
\hline Duddela, Varaprasad & 1 & 3 \\
\hline Dutta, Mithun & 1 & 3 \\
\hline Edjlali, Ladan & 1 & 3 \\
\hline Eftekhari, Aziz & 1 & 3 \\
\hline Eker, Tulin & 1 & 3 \\
\hline El-Mekabaty, Ahmed & 1 & 3 \\
\hline Elsefy, Doaa E. & 1 & 3 \\
\hline Fakhry, Ahmed & 1 & 3 \\
\hline Fatonah, Ani & 1 & 3 \\
\hline Fatyeyeva, Kateryna & 1 & 3 \\
\hline
\end{tabular}




\begin{tabular}{|c|c|c|}
\hline Author & Documents & Citations \\
\hline Forouzanfar, Fateme & 1 & 3 \\
\hline Gadalla, Mamdouh A. & 1 & 3 \\
\hline Gollapudi, Venkata Ramanamurthy & 1 & 3 \\
\hline Gouthami, Thumma & 1 & 3 \\
\hline Grimme, Marc & 1 & 3 \\
\hline Gusdinar, Tutus & 1 & 3 \\
\hline Guyo, Upenyu & 1 & 3 \\
\hline Hadi, Angham G. & 1 & 3 \\
\hline Hairunisa, Nany & 1 & 3 \\
\hline Hajibonabi, Farid & 1 & 3 \\
\hline Hananelhaes & 1 & 3 \\
\hline Harkov, Stefan & 1 & 3 \\
\hline Hasan, Md Rakibul & 1 & 3 \\
\hline Hassan, Md Mehedi & 1 & 3 \\
\hline Hassanein, Abd-Elatif A. & 1 & 3 \\
\hline Heravi, Mohammad M. & 1 & 3 \\
\hline Hosen, Md Sazib & 1 & 3 \\
\hline Inamuddin & 1 & 3 \\
\hline Ing, Alex Wong Chung & 1 & 3 \\
\hline Islam, Md Rakibul & 1 & 3 \\
\hline Ismail, Bushra & 1 & 3 \\
\hline Issaoui, Noureddine & 1 & 3 \\
\hline Jafari, Samira & 1 & 3 \\
\hline Jorge, Juliana & 1 & 3 \\
\hline Kadhom, Mohammed & 1 & 3 \\
\hline Kanchi, Suvardhan & 1 & 3 \\
\hline Kandil, E. M. & 1 & 3 \\
\hline Karimi, Abbas & 1 & 3 \\
\hline Karimian, Ramin & 1 & 3 \\
\hline Kaya, Harun & 1 & 3 \\
\hline Khan, Anish & 1 & 3 \\
\hline Khanmohammadi, Sina & 1 & 3 \\
\hline Khatab, Tamer K. & 1 & 3 \\
\hline Kiran, Gangarapu & 1 & 3 \\
\hline Koun, Malys & 1 & 3 \\
\hline Kulmetyeva, Margarita Anatolyevna & 1 & 3 \\
\hline Lotfipour, Farzaneh & 1 & 3 \\
\hline Mahmoud, Abd Elaziz & 1 & 3 \\
\hline Mahmud, Shahin & 1 & 3 \\
\hline Maleki, Abdollah & 1 & 3 \\
\hline Maleki, Mohammad Jafar & 1 & 3 \\
\hline Mallavarapu, Umamahesh & 1 & 3 \\
\hline Marais, Stephane & 1 & 3 \\
\hline Marcano, Aracelys & 1 & 3 \\
\hline Mehrabani, Mojtaba Ghanbari & 1 & 3 \\
\hline Mele, Altin & 1 & 3 \\
\hline Mignoni, Marcelo Luis & 1 & 3 \\
\hline Miloudi, Safia & 1 & 3 \\
\hline Minakova, Mariya Yur'evna & 1 & 3 \\
\hline Miroshnichenko, Larisa Arkad'evna & 1 & 3 \\
\hline Mohafrash, Samia M. & 1 & 3 \\
\hline Mohamed, Doha Adel Awies & 1 & 3 \\
\hline Mohammadabadi, Rezvan & 1 & 3 \\
\hline Mohammadi, Seyed Abolghasem & 1 & 3 \\
\hline Mohammed, Haitham & 1 & 3 \\
\hline Moreira Ficanha, Aline Matuella & 1 & 3 \\
\hline Morsali, Ali & 1 & 3 \\
\hline Mossa, Abdel-Twab H. & 1 & 3 \\
\hline Mostafidi, Elmira & 1 & 3 \\
\hline Muchanyereyi, Netai & 1 & 3 \\
\hline Ngwenya, Sithulisiwe & 1 & 3 \\
\hline
\end{tabular}




\begin{tabular}{|c|c|c|}
\hline Author & Documents & Citations \\
\hline Noureddine, Olfa & 1 & 3 \\
\hline Nyamunda, Benias Chomunorwa & 1 & 3 \\
\hline Pamungkas, Wilis Okti & 1 & 3 \\
\hline Polykova, Tatyana Yur'evna & 1 & 3 \\
\hline Porumbel, Iuliana & 1 & 3 \\
\hline Pourhassan-Moghaddam, Mohammad & 1 & 3 \\
\hline Prajapati, Anjali & 1 & 3 \\
\hline Pramanik, Panchanan & 1 & 3 \\
\hline Prasad, Devarakonda Krishna & 1 & 3 \\
\hline Rahmouni, Abdelkader & 1 & 3 \\
\hline Rehman, Saeed & 1 & 3 \\
\hline Rezaie, Fereshte & 1 & 3 \\
\hline Roshanravan, Babak & 1 & 3 \\
\hline Ruscuklu, Dane & 1 & 3 \\
\hline Salatin, Sara & 1 & 3 \\
\hline Salim, Sa'diah & 1 & 3 \\
\hline Samani, Amir Abbas & 1 & 3 \\
\hline Samiei, Mohammad & 1 & 3 \\
\hline Samrot, Antony V. & 1 & 3 \\
\hline Seetha, Jaswanth & 1 & 3 \\
\hline Shah, Jehanzeb Ali & 1 & 3 \\
\hline Shahzad, Muhammad & 1 & 3 \\
\hline Sharaawy, Sahar & 1 & 3 \\
\hline Shelepeten, Lesya & 1 & 3 \\
\hline Shukri, Harvie & 1 & 3 \\
\hline Simanina, Elena Vladislavovna & 1 & 3 \\
\hline Stavrova, Larisa Aleksandrovna & 1 & 3 \\
\hline Suslov, Nikolay Innokent'evich & 1 & 3 \\
\hline Tanomand, Asghar & 1 & 3 \\
\hline Tariq, Madiha & 1 & 3 \\
\hline Udut, Elena Vladimirovna & 1 & 3 \\
\hline Udut, Vladimir Vasil'evich & 1 & 3 \\
\hline Ullah, Aziz & 1 & 3 \\
\hline Uslu, Mehmet Emin & 1 & 3 \\
\hline Vatti, Chandra Sekhar & 1 & 3 \\
\hline Verelst, Marc & 1 & 3 \\
\hline Wicaksono, Ig Satrio & 1 & 3 \\
\hline Yilmazer, Ozgur & 1 & 3 \\
\hline Yousef, Tarek Ahmed & 1 & 3 \\
\hline Zarghami, Nosratollah & 1 & 3 \\
\hline Zhang, Shaoqing & 1 & 3 \\
\hline Zhdanov, Vadim Vadimovich & 1 & 3 \\
\hline Zinyama, Ngceboyakwethu Primrose & 1 & 3 \\
\hline Zyuz'kov, Gleb Nikolaevich & 1 & 3 \\
\hline Regina, Cauich-Carrillo & 13 & 2 \\
\hline Lenin, Hau-Heredia & 8 & 2 \\
\hline Jhair, Cabrera-Tuz & 7 & 2 \\
\hline Elodia, Garcia-Cervera & 6 & 2 \\
\hline Gomez Eduardo, Pool & 6 & 2 \\
\hline Malviya, Rishabha & 5 & 2 \\
\hline Rolando, Garcia-Martinez & 5 & 2 \\
\hline Qayyum, Muhammad Abdul & 4 & 2 \\
\hline Lubenets, Vira & 3 & 2 \\
\hline Manjunatha, $\mathrm{H}$. & 3 & 2 \\
\hline Martinez, Karina D. & 3 & 2 \\
\hline Mattaparthi, Venkata Satish Kumar & 3 & 2 \\
\hline Ramesh, S. & 3 & 2 \\
\hline Ratnam, K. Venkata & 3 & 2 \\
\hline Savin, Ioana & 3 & 2 \\
\hline Ali, Farida A. & 2 & 2 \\
\hline Bigdeli, Tania & 2 & 2 \\
\hline
\end{tabular}




\begin{tabular}{|c|c|c|}
\hline Author & Documents & Citations \\
\hline Das, Alok Prasad & 2 & 2 \\
\hline Das, Dipak Kumar & 2 & 2 \\
\hline Foroughifar, Neda & 2 & 2 \\
\hline Gamiotea-Turro, Daylin & 2 & 2 \\
\hline Guerrero, Jose & 2 & 2 \\
\hline Heydari, Reza & 2 & 2 \\
\hline Khorshidian, Nasim & 2 & 2 \\
\hline Kumar, Yogendra & 2 & 2 \\
\hline Kumarasamy, Suresh & 2 & 2 \\
\hline Marcus, Iuliana & 2 & 2 \\
\hline Mishra, Dilip Kumar & 2 & 2 \\
\hline Narayanan, Mathiyazhagan & 2 & 2 \\
\hline Norouzi, Nima & 2 & 2 \\
\hline Prakash, P. & 2 & 2 \\
\hline Ranganathan, Muthusamy & 2 & 2 \\
\hline Rodriguez-Chanfrau, Jorge Enrique & 2 & 2 \\
\hline Shah, Muzammil & 2 & 2 \\
\hline Shahouzehi, Beydolah & 2 & 2 \\
\hline Suhartono, Suhartono & 2 & 2 \\
\hline Tahvildari, Kambiz & 2 & 2 \\
\hline Talebi, Saeed & 2 & 2 \\
\hline Valduga, Eunice & 2 & 2 \\
\hline Abirami, S. & 1 & 2 \\
\hline Abotbina, Ismail Miftah & 1 & 2 \\
\hline Abu-Rayyan, Ahmed & 1 & 2 \\
\hline Abuelazm, Mohamed Gaber & 1 & 2 \\
\hline Acharya, Rashmi & 1 & 2 \\
\hline Aghaei, Hossein & 1 & 2 \\
\hline Ahmad, Mohd Azlan & 1 & 2 \\
\hline Ahmadian, Salman & 1 & 2 \\
\hline Ahmed, Haseen & 1 & 2 \\
\hline Ahmed, Hend & 1 & 2 \\
\hline Al-Anber, Zaid & 1 & 2 \\
\hline Al-Momani, Idrees & 1 & 2 \\
\hline Ali, Kashif & 1 & 2 \\
\hline Ali, Shaista & 1 & 2 \\
\hline Almogbel, Mohammed S. & 1 & 2 \\
\hline Amri, Mohamed & 1 & 2 \\
\hline Apsari, Cynthia Nurul & 1 & 2 \\
\hline Arshad, Muhammad & 1 & 2 \\
\hline Asghari, Somayeh & 1 & 2 \\
\hline Avunduk, Mustafa Cihat & 1 & 2 \\
\hline Bagheri, Samira & 1 & 2 \\
\hline Bahrami, Mohammad Kazem & 1 & 2 \\
\hline Bahrizadeh, Shiva & 1 & 2 \\
\hline Balachandran, Manoj & 1 & 2 \\
\hline Balev, Dessislav & 1 & 2 \\
\hline Barar, Jaleh & 1 & 2 \\
\hline Bare, Yohanes & 1 & 2 \\
\hline Barroso, Ana Rey & 1 & 2 \\
\hline Basyouni, Osama H. & 1 & 2 \\
\hline Ben Khemis, Ines & 1 & 2 \\
\hline Besbes, Nadia & 1 & 2 \\
\hline Biswas, Rajib & 1 & 2 \\
\hline Bittante, Ana Monica Q. B. & 1 & 2 \\
\hline Borah, Priyanka & 1 & 2 \\
\hline Bose, Gouranga & 1 & 2 \\
\hline Brandan, Silvia Antonia & 1 & 2 \\
\hline Butko, Yaroslava & 1 & 2 \\
\hline Cin, Gunseli Turgut & 1 & 2 \\
\hline Cirpici, Ioana & 1 & 2 \\
\hline
\end{tabular}




\begin{tabular}{|c|c|c|}
\hline Author & Documents & Citations \\
\hline Corbu, Viorica & 1 & 2 \\
\hline Dacha, Preethi & 1 & 2 \\
\hline Dan, Nily & 1 & 2 \\
\hline Dang, Phuong T. & 1 & 2 \\
\hline Dastjerdi, Roya & 1 & 2 \\
\hline Davoodi, Seyed Dariush & 1 & 2 \\
\hline De Andrade Junior, Pedro Paulo & 1 & 2 \\
\hline De Camargo, Emerson Rodrigues & 1 & 2 \\
\hline De Queiroz, Daniely Ferreira & 1 & 2 \\
\hline Dehghanzadeh, Salva & 1 & 2 \\
\hline Deng, Taoli & 1 & 2 \\
\hline Dragoev, Stefan & 1 & 2 \\
\hline Ducu, Robert & 1 & 2 \\
\hline Duvoisin, Charles Adriano & 1 & 2 \\
\hline El-Gammal, Ola Ahmed & 1 & 2 \\
\hline El-Sayed, El Sayed M. & 1 & 2 \\
\hline Elizabeth, Agustina & 1 & 2 \\
\hline Eslami, Gilda & 1 & 2 \\
\hline Esmaeilzadeh, Feridun & 1 & 2 \\
\hline Farrukh, Muhammad Akhyar & 1 & 2 \\
\hline Fatahpour, Maryam & 1 & 2 \\
\hline Fathirad, Fariba & 1 & 2 \\
\hline Fazaeli, Reza & 1 & 2 \\
\hline Fedorova, Olena & 1 & 2 \\
\hline Feilizadeh, Mansoor & 1 & 2 \\
\hline Flaker, Christian H. C. & 1 & 2 \\
\hline Gad, Ehab Said & 1 & 2 \\
\hline Gamal, Fatma & 1 & 2 \\
\hline Gerrard, David & 1 & 2 \\
\hline Ghanem, Alia S. A-E. & 1 & 2 \\
\hline Ghasemi, Younes & 1 & 2 \\
\hline Ghita, Alexandra & 1 & 2 \\
\hline Gholamhoseinian, Ahmad & 1 & 2 \\
\hline Gohari, Gholamreza & 1 & 2 \\
\hline Gola, Deepak & 1 & 2 \\
\hline Gupta, Dhriti & 1 & 2 \\
\hline Gururani, Prateek & 1 & 2 \\
\hline Haghani-Haghighi, Hameed & 1 & 2 \\
\hline Hajimohammadi, Bahador & 1 & 2 \\
\hline Halevas, Eleftherios G. & 1 & 2 \\
\hline Hassanpouraghdam, Mohammad Bagher & 1 & 2 \\
\hline Helmy, Samah A. & 1 & 2 \\
\hline Hodges, Deidra R. & 1 & 2 \\
\hline Horst, Jose Diogo & 1 & 2 \\
\hline Hosseini, Saeede Sadat & 1 & 2 \\
\hline Ibrahim, Ahmed H. & 1 & 2 \\
\hline Ivanova, Sonya & 1 & 2 \\
\hline Jabbarzare, Saeid & 1 & 2 \\
\hline Jaratrungtawee, Amornmart & 1 & 2 \\
\hline Jarukumjorn, Kasama & 1 & 2 \\
\hline Joffraud, Jean-Jacques & 1 & 2 \\
\hline Jyoti & 1 & 2 \\
\hline Kamilla, Sushanta K. & 1 & 2 \\
\hline Kanwal, Zobia & 1 & 2 \\
\hline Karami, Soghra & 1 & 2 \\
\hline Karmakar, Pralay Kumar & 1 & 2 \\
\hline Kartawinata, Tutus Gusdinar & 1 & 2 \\
\hline Khammee, Thongchai & 1 & 2 \\
\hline Khan, Fahad & 1 & 2 \\
\hline Khojastehnezhad, Amir & 1 & 2 \\
\hline Kolahdouzan, Masoud & 1 & 2 \\
\hline
\end{tabular}




\begin{tabular}{|c|c|c|}
\hline Author & Documents & Citations \\
\hline Konovalova, Svitlana & 1 & 2 \\
\hline Krishnan, Ramya & 1 & 2 \\
\hline Krisnamurti, Gabriela Candrakirana & 1 & 2 \\
\hline Krychkovska, Aelita & 1 & 2 \\
\hline Kumar, Anil & 1 & 2 \\
\hline Kuno, Mayuso & 1 & 2 \\
\hline Kuz'menko, Lyudmyla & 1 & 2 \\
\hline Kuznetsova, Irina M. & 1 & 2 \\
\hline Lau, Kevin Beng Kieng & 1 & 2 \\
\hline Lavysh, Andrei V. & 1 & 2 \\
\hline Le, Giang H. & 1 & 2 \\
\hline Lee, Gun D. & 1 & 2 \\
\hline Levashova, Olha & 1 & 2 \\
\hline $\mathrm{Li}, \mathrm{Hu}$ & 1 & 2 \\
\hline Lisar, Seyed Yahya Salehi & 1 & 2 \\
\hline Lobo, Jumiarti Andi & 1 & 2 \\
\hline $\begin{array}{llll}\text { Lorenza, Margaretha } & \text { Rika } & \text { Wahyusari } \\
\text { Gabrella } & & & \\
\end{array}$ & 1 & 2 \\
\hline Lourenco, Rodrigo V. & 1 & 2 \\
\hline Lugovskii, Alexander A. & 1 & 2 \\
\hline Madam, Marzieh Sadat & 1 & 2 \\
\hline Madkour, Tarek M. & 1 & 2 \\
\hline Mahdavinia, Gholam Reza & 1 & 2 \\
\hline Maleki, Behrooz & 1 & 2 \\
\hline Maleki-Dizaj, Solmaz & 1 & 2 \\
\hline Malik, Shahana & 1 & 2 \\
\hline Mandour, Sherif Abdelmoneam & 1 & 2 \\
\hline Marpongahtun, Marpongahtun & 1 & 2 \\
\hline Maskevich, Alexander A. & 1 & 2 \\
\hline Massoud, Ramona & 1 & 2 \\
\hline Maurady, Amal & 1 & 2 \\
\hline Menazea, A. A. & 1 & 2 \\
\hline Mishra, Rashmi & 1 & 2 \\
\hline Mishra, Roshni & 1 & 2 \\
\hline Mofrad, Akram Vedad & 1 & 2 \\
\hline Mohamed, Aya A. & 1 & 2 \\
\hline Mohammadi, Abdorreza & 1 & 2 \\
\hline Mohammadi, Gholamabbas & 1 & 2 \\
\hline Mohammadi, Masoumeh & 1 & 2 \\
\hline Mondal, Soumila & 1 & 2 \\
\hline Monka, Natalija & 1 & 2 \\
\hline Mostafavi, Ali & 1 & 2 \\
\hline Mowla, Dariush & 1 & 2 \\
\hline Najee, Haifaa & 1 & 2 \\
\hline Nakev, Jivko & 1 & 2 \\
\hline Nguyen, Manh B. & 1 & 2 \\
\hline Nikolova, Tanya & 1 & 2 \\
\hline Ninsuwan, Unchalee & 1 & 2 \\
\hline Ochoa, Katia & 1 & 2 \\
\hline Oktaviary, Rozana & 1 & 2 \\
\hline Omara, Wessam & 1 & 2 \\
\hline Omidi, Yadollah & 1 & 2 \\
\hline Orlov, Yuriy L. & 1 & 2 \\
\hline Ozturk, Suat & 1 & 2 \\
\hline Pantazaki, Anastasia A. & 1 & 2 \\
\hline Parida, Kulamani & 1 & 2 \\
\hline Pathak, Jainendra & 1 & 2 \\
\hline Pham, Giang T. T. & 1 & 2 \\
\hline Pham, Thu Thi & 1 & 2 \\
\hline Phoonan, Wachiraporn & 1 & 2 \\
\hline Pircalabioru, Gratiela Gradisteanu & 1 & 2 \\
\hline
\end{tabular}




\begin{tabular}{|c|c|c|}
\hline Author & Documents & Citations \\
\hline Plengnok, Usasawan & 1 & 2 \\
\hline Qayyum, Abdul & 1 & 2 \\
\hline Rachmad, Yoga Tribakti & 1 & 2 \\
\hline Ragavi, R. & 1 & 2 \\
\hline Rahman, Taj Ur & 1 & 2 \\
\hline Rajneesh & 1 & 2 \\
\hline Ramana, Ch. V. V. & 1 & 2 \\
\hline Rodriguez-Riera, Zalua & 1 & 2 \\
\hline Sadeghi, Behnam & 1 & 2 \\
\hline Sadeh, Fatemeh Noori & 1 & 2 \\
\hline Sadok, Saloua & 1 & 2 \\
\hline Saghavaz, Bahare Hedayati & 1 & 2 \\
\hline Sahin, Yesim Muge & 1 & 2 \\
\hline Sahna, Engin & 1 & 2 \\
\hline Sajid, Muhammad & 1 & 2 \\
\hline Salim, Mohd Razman & 1 & 2 \\
\hline Salmiati, Salmiati & 1 & 2 \\
\hline Sarfraz, Raja Adil & 1 & 2 \\
\hline Sari, Dewi Ratih Tirto & 1 & 2 \\
\hline Shahi, Shahriar & 1 & 2 \\
\hline Shamspur, Tayebeh & 1 & 2 \\
\hline Shehata, Dina & 1 & 2 \\
\hline Shojaee, Asma & 1 & 2 \\
\hline Shojaee-Aliabadi, Saeedeh & 1 & 2 \\
\hline Siabi, Shadi & 1 & 2 \\
\hline Singh, Prabal P. & 1 & 2 \\
\hline Singh, Shailendra P. & 1 & 2 \\
\hline Sinha, Rajeshwar P. & 1 & 2 \\
\hline Sobral, Paulo J. A. & 1 & 2 \\
\hline Subedi, Mahendra & 1 & 2 \\
\hline Sukrasno & 1 & 2 \\
\hline Sulatskaya, Anna I. & 1 & 2 \\
\hline Tawfik, Walid & 1 & 2 \\
\hline Tishakova, Tetyana & 1 & 2 \\
\hline Tkachova, Oksana & 1 & 2 \\
\hline Topel, Onder & 1 & 2 \\
\hline Topel, Seda Demirel & 1 & 2 \\
\hline Tran, Hoa T. K. & 1 & 2 \\
\hline Turoverov, Konstantin K. & 1 & 2 \\
\hline Tyagi, Pankaj K. & 1 & 2 \\
\hline Ulanova, Vera & 1 & 2 \\
\hline Vashishtha, Vinod Kumar & 1 & 2 \\
\hline Vasylyuk, Sofiya & 1 & 2 \\
\hline Vieira, Rogerio De Almeida & 1 & 2 \\
\hline Vlahova-Vangelova, Dessislava & 1 & 2 \\
\hline Vlaskin, M. S. & 1 & 2 \\
\hline Voropay, Evgeniy S. & 1 & 2 \\
\hline Vu, Loi D. & 1 & 2 \\
\hline Vu, Tuan A. & 1 & 2 \\
\hline Wiwanitkit, Viroj & 1 & 2 \\
\hline Yasri, Sora & 1 & 2 \\
\hline Yazgan, Betul & 1 & 2 \\
\hline Yetmez, Mehmet & 1 & 2 \\
\hline Zeb, Muhammad Aurang & 1 & 2 \\
\hline Zhang, Qiuyun & 1 & 2 \\
\hline Zhang, Yutao & 1 & 2 \\
\hline Zhao, Li & 1 & 2 \\
\hline Ziglari, Alireza & 1 & 2 \\
\hline Zikry, Amina A. F. & 1 & 2 \\
\hline Zoghi, Alaleh & 1 & 2 \\
\hline Francisco, Diaz-Cedillo & 12 & 1 \\
\hline
\end{tabular}




\begin{tabular}{|c|c|c|}
\hline Author & Documents & Citations \\
\hline Eduardo, Pool-Gomez & 5 & 1 \\
\hline Patricia, Hernandez-Vasquez & 5 & 1 \\
\hline Alondra, Alfonso-Jimenez & 4 & 1 \\
\hline Ionescu, Bogdan & 4 & 1 \\
\hline Ionescu, Diana & 4 & 1 \\
\hline Khalil, Ahmed M. & 4 & 1 \\
\hline Laura, Benitez-Coeto & 4 & 1 \\
\hline Raquel, Estrella-Barron & 4 & 1 \\
\hline Singh, Sulochana & 4 & 1 \\
\hline Yaritza, Borges-Ballote & 4 & 1 \\
\hline Aggarwal, Babita & 3 & 1 \\
\hline Ajeet & 3 & 1 \\
\hline Arba, Muhammad & 3 & 1 \\
\hline Arsene, Cosmin & 3 & 1 \\
\hline Bleotu, Coralia & 3 & 1 \\
\hline Cedillo Francisco, Diaz & 3 & 1 \\
\hline Cucu, Natalia & 3 & 1 \\
\hline Dalai, Biswajit & 3 & 1 \\
\hline Konovalova, Svetlana & 3 & 1 \\
\hline Logoyda, Liliya S. & 3 & 1 \\
\hline Perla, Parra-Galindo & 3 & 1 \\
\hline Sheremet, Michael, I & 3 & 1 \\
\hline Singh, Inderbir & 3 & 1 \\
\hline Singh, Saroj Kumar & 3 & 1 \\
\hline Socorro, Herrera-Meza & 3 & 1 \\
\hline Sukla, Lala Behari & 3 & 1 \\
\hline Talukdar, Malabika & 3 & 1 \\
\hline Abaszadeh, Mehdi & 2 & 1 \\
\hline Abou El Ela, Amal El Sayeh F. & 2 & 1 \\
\hline Akhtar, Javeed & 2 & 1 \\
\hline Alejandra, Garcimarero-Espino E. & 2 & 1 \\
\hline Altin, Serdar & 2 & 1 \\
\hline Ashrafi, Hajar & 2 & 1 \\
\hline Azadi, Amir & 2 & 1 \\
\hline Bankupalli, Satyavathi & 2 & 1 \\
\hline Bohlouli, Sepideh & 2 & 1 \\
\hline Casu, Cinzia & 2 & 1 \\
\hline Dash, Sarat Kumar & 2 & 1 \\
\hline Demirel, Serkan & 2 & 1 \\
\hline Denti, Andressa Franco & 2 & 1 \\
\hline Duta, Maria & 2 & 1 \\
\hline Dzantiev, Boris B. & 2 & 1 \\
\hline El Khatib, Mona Mohamed & 2 & 1 \\
\hline Eskandari, Mahnaz & 2 & 1 \\
\hline Gangarapu, Kiran & 2 & 1 \\
\hline Ghavimi, Mohammad Ali & 2 & 1 \\
\hline Ghosh, Anuja & 2 & 1 \\
\hline Ghosh, Manas & 2 & 1 \\
\hline Gilca, Ramona & 2 & 1 \\
\hline Javad, Pazhayakath Thevarkattil Mohamed & 2 & 1 \\
\hline Kamalak, Hakan & 2 & 1 \\
\hline Kar, Prativa & 2 & 1 \\
\hline Mandapati, Ramesh Naidu & 2 & 1 \\
\hline Maria, Cristina & 2 & 1 \\
\hline Mazutti, Marcio A. & 2 & 1 \\
\hline Melnyk, Nataliia A. & 2 & 1 \\
\hline Neacsu, Gabriela & 2 & 1 \\
\hline Nikmatin, Siti & 2 & 1 \\
\hline Oz, Erdinc & 2 & 1 \\
\hline Paduraru, Dan Nicolae & 2 & 1 \\
\hline Pasdaran, Ardalan & 2 & 1 \\
\hline
\end{tabular}




\begin{tabular}{|c|c|c|}
\hline Author & Documents & Citations \\
\hline Pidruchna, Svitlana R. & 2 & 1 \\
\hline Pilosof, Ana M. R. & 2 & 1 \\
\hline Ponnam, Vijetha & 2 & 1 \\
\hline Prabhakar, Pranav Kumar & 2 & 1 \\
\hline Purayil, Sajna Keeyari & 2 & 1 \\
\hline Sacagiu, Bogdan & 2 & 1 \\
\hline Saidy, Euan-Hau & 2 & 1 \\
\hline Samal, D. P. Krishna & 2 & 1 \\
\hline Schmaltz, Silvana & 2 & 1 \\
\hline Selamoglu, Zeliha & 2 & 1 \\
\hline Selvarani, Jenifer A. & 2 & 1 \\
\hline Sharma, Akanksha & 2 & 1 \\
\hline Sharma, Pramod Kumar & 2 & 1 \\
\hline Shiprath, Kudekallu & 2 & 1 \\
\hline Soman, Somy & 2 & 1 \\
\hline Tomas, Lopez-Gutierez & 2 & 1 \\
\hline Tres, Bethina Pascuetti & 2 & 1 \\
\hline Vigano, Luca & 2 & 1 \\
\hline Yazmin, Ortiz-Ake & 2 & 1 \\
\hline Zendeboodi, Fatemeh & 2 & 1 \\
\hline Zherdev, Anatoly, V & 2 & 1 \\
\hline Aamir, Muhammad & 1 & 1 \\
\hline Abbas, Hazim F. & 1 & 1 \\
\hline Abbasi, Amin & 1 & 1 \\
\hline Abd Ali, Ali & 1 & 1 \\
\hline Abd El-Salam, Mohamed H. & 1 & 1 \\
\hline Abd Elaziz, Mariam Sayed & 1 & 1 \\
\hline Abd-Ellatef, Sarah A. & 1 & 1 \\
\hline Abdel-Galil, Ebrahim & 1 & 1 \\
\hline Abdullah, Noorlidah & 1 & 1 \\
\hline Abiras, Hadjer W. & 1 & 1 \\
\hline Adeiza, Otohinoyi David & 1 & 1 \\
\hline Adenike, Sulaiman Faoziyat & 1 & 1 \\
\hline Afanasyev, Ilya I. & 1 & 1 \\
\hline Afchangi, Leila & 1 & 1 \\
\hline Afolabi, Sulaiman Abdulfattah & 1 & 1 \\
\hline Afsahi, Mohammad Mehdi & 1 & 1 \\
\hline Agag, Dina A. & 1 & 1 \\
\hline Agarwal, Prachi & 1 & 1 \\
\hline Ahmed, Shedid S. & 1 & 1 \\
\hline Ahyat, Norhidayah & 1 & 1 \\
\hline Ajobola, Iyiola Oluyinka & 1 & 1 \\
\hline Akhter, Shirin & 1 & 1 \\
\hline Aksenova, Elena A. & 1 & 1 \\
\hline Akyol, Emel & 1 & 1 \\
\hline Al Riami, Khamis & 1 & 1 \\
\hline Al-Quaderi, Golam Dastegir & 1 & 1 \\
\hline Albrakati, Ashraf & 1 & 1 \\
\hline Alcocer-Vallejo, Robert & 1 & 1 \\
\hline Alexander, Anila K. & 1 & 1 \\
\hline Alexandrescu, Lygia & 1 & 1 \\
\hline Alexandrovna, Dobrovolskaya Tatiana & 1 & 1 \\
\hline Alharbi, Najeeb & 1 & 1 \\
\hline Ali, Murad & 1 & 1 \\
\hline Alsamadany, Hameed & 1 & 1 \\
\hline Alshihi, Hajer & 1 & 1 \\
\hline Alzahrani, Yahya & 1 & 1 \\
\hline Aminudin, Norhaniza & 1 & 1 \\
\hline Amirabadi, Parvin Eslami & 1 & 1 \\
\hline Anand, Kiran Kumar Hoskote & 1 & 1 \\
\hline Anchuri, Shyam Sunder & 1 & 1 \\
\hline
\end{tabular}




\begin{tabular}{|c|c|c|}
\hline Author & Documents & Citations \\
\hline Andac, Muge & 1 & 1 \\
\hline Andrei, Graciela & 1 & 1 \\
\hline Aniya, Vineet & 1 & 1 \\
\hline Ardalan, Mohammadreza & 1 & 1 \\
\hline Ariotti, Gustavo & 1 & 1 \\
\hline Arteaga-Robalino, Andrea & 1 & 1 \\
\hline Asadzadeh, Sepideh & 1 & 1 \\
\hline Ashyuce, Sevgi & 1 & 1 \\
\hline Asl, Mir Mehdi Chinifroush & 1 & 1 \\
\hline Awadallah, Omima A. & 1 & 1 \\
\hline Ayaad, Dalia M. & 1 & 1 \\
\hline Ayoubi, Mahdi & 1 & 1 \\
\hline Azadeh, Mehdi & 1 & 1 \\
\hline Azmi, Alyza A. & 1 & 1 \\
\hline Azzam, Rasha A. & 1 & 1 \\
\hline Babu, Kothamasu Suresh & 1 & 1 \\
\hline Badr, Shalabya, I & 1 & 1 \\
\hline Baghel, Dileep Singh & 1 & 1 \\
\hline Bakshi, Herman & 1 & 1 \\
\hline Bal, Celal & 1 & 1 \\
\hline Balivand, Zohreh & 1 & 1 \\
\hline Balzarini, Jan & 1 & 1 \\
\hline Banaei, Shokofeh & 1 & 1 \\
\hline Barghi, Leila & 1 & 1 \\
\hline Barounian, Mohammad Hasan & 1 & 1 \\
\hline Bartok, Ruxandra & 1 & 1 \\
\hline Bashir, Shubli & 1 & 1 \\
\hline Bathla, Angel & 1 & 1 \\
\hline Batool, Madiha & 1 & 1 \\
\hline Bektas, Hatice & 1 & 1 \\
\hline Belarbi, L. & 1 & 1 \\
\hline Bennabi, Fatima & 1 & 1 \\
\hline Bennabi, Lamia & 1 & 1 \\
\hline Bera, Aindrila & 1 & 1 \\
\hline Bereli, Nilay & 1 & 1 \\
\hline Bernardi, Julia Lisboa & 1 & 1 \\
\hline Bgatova, Natalia & 1 & 1 \\
\hline Bhuiyan, Mahabub Alam & 1 & 1 \\
\hline Biswal, Prasandeep & 1 & 1 \\
\hline Bodron, Paul & 1 & 1 \\
\hline Bondarenko, Natalia & 1 & 1 \\
\hline Botezatu, Anca & 1 & 1 \\
\hline Botlagunta, Mahendran & 1 & 1 \\
\hline Burlibasa, Liliana & 1 & 1 \\
\hline Calderon-Pardo, Jose & 1 & 1 \\
\hline Capikova, Jana & 1 & 1 \\
\hline Castellano, Carlo & 1 & 1 \\
\hline Castillo-Cabay, Luis & 1 & 1 \\
\hline Cenet, Menderes & 1 & 1 \\
\hline Cetin, Kemal & 1 & 1 \\
\hline Chaibi, Wahiba & 1 & 1 \\
\hline Chandra, Sharath S. P. & 1 & 1 \\
\hline Chirila, Mihaela & 1 & 1 \\
\hline Choudhury, Shamima & 1 & 1 \\
\hline Colet, Rosicler & 1 & 1 \\
\hline Cristea, Violeta & 1 & 1 \\
\hline Da Costa, Liovando M. & 1 & 1 \\
\hline Da Costa, Marcelo R. & 1 & 1 \\
\hline Da Cruz, Adriano Gomes & 1 & 1 \\
\hline Damilare, Rotimi & 1 & 1 \\
\hline Daoush, Walid Mohamed & 1 & 1 \\
\hline
\end{tabular}




\begin{tabular}{|c|c|c|}
\hline Author & Documents & Citations \\
\hline Daryakenari, Narmi & 1 & 1 \\
\hline Dash, Aditya Kishore & 1 & 1 \\
\hline De Almeida, Thiago C. & 1 & 1 \\
\hline De Clercq, Erik & 1 & 1 \\
\hline De Medeiros Burkert, Janaina Fernandes & 1 & 1 \\
\hline De Mello, Vanessa J. & 1 & 1 \\
\hline De Oliveira, Tania T. & 1 & 1 \\
\hline De Sousa Filho, Paulo Cesar & 1 & 1 \\
\hline Desiateryk, Volodimir, I & 1 & 1 \\
\hline Dhal, Kshirabdhitanaya & 1 & 1 \\
\hline Dhiva, S. & 1 & 1 \\
\hline Dhurai, Bhaarathi & 1 & 1 \\
\hline Din, Mohy Ud & 1 & 1 \\
\hline Ditu, Lia Mara & 1 & 1 \\
\hline Doddarevanna, Revanasiddappa Hosakere & 1 & 1 \\
\hline Dordevic, Dani & 1 & 1 \\
\hline Dulgerbaki, Cigdem & 1 & 1 \\
\hline Dziubanovskyi, Ihor Ya & 1 & 1 \\
\hline Ehsani, Mohammad Reza & 1 & 1 \\
\hline El Gabaly, Shimaa G. & 1 & 1 \\
\hline El Idrissi, Brahim Chafik & 1 & 1 \\
\hline El Yacoubi, Ahmed & 1 & 1 \\
\hline El-Ghamry, Abbas A. & 1 & 1 \\
\hline El-Hawary, Ahmad S. & 1 & 1 \\
\hline El-Kohadary, Sherif & 1 & 1 \\
\hline El-Saber, Batiha Gaber & 1 & 1 \\
\hline El-Sayed, Mohamed H. & 1 & 1 \\
\hline El-Shanshoury, Abd El-Raheem R. & 1 & 1 \\
\hline El-Shibiny, Safinaz & 1 & 1 \\
\hline Elbiomy, Sarah A. & 1 & 1 \\
\hline Elizabeth, Montano-Tapia & 1 & 1 \\
\hline Elsehemy, Islam A. & 1 & 1 \\
\hline Eren, Esin & 1 & 1 \\
\hline Esmaeili, Saeideh & 1 & 1 \\
\hline Etemadi, Farzin & 1 & 1 \\
\hline Fabi, Maryam & 1 & 1 \\
\hline Fadda, Ahmed A. & 1 & 1 \\
\hline Faiz-Ul-Hassan & 1 & 1 \\
\hline Fan, Zheng & 1 & 1 \\
\hline Farahmand, Sara & 1 & 1 \\
\hline Farajzadeh, Aysan & 1 & 1 \\
\hline Farsi, Ali & 1 & 1 \\
\hline Farsi, Milad & 1 & 1 \\
\hline Fawzy, Badie M. & 1 & 1 \\
\hline Fazli, Mostafa & 1 & 1 \\
\hline Felitti, Rafael & 1 & 1 \\
\hline Ferraro, Angelo & 1 & 1 \\
\hline Ferretti, Marco & 1 & 1 \\
\hline Filardi, Marcelo A. & 1 & 1 \\
\hline Filibus, Maimako Rotdelmwa & 1 & 1 \\
\hline Foletto, Edson L. & 1 & 1 \\
\hline Fusinato, Veronica Baldon & 1 & 1 \\
\hline Gaber, Heba A. & 1 & 1 \\
\hline Galvao Silva, Katleyn Polizeli & 1 & 1 \\
\hline Garg, Kanika & 1 & 1 \\
\hline Gautam, Pankaj & 1 & 1 \\
\hline Gayeski, Luana & 1 & 1 \\
\hline George, Mathew & 1 & 1 \\
\hline Ghadam, Parinaz & 1 & 1 \\
\hline Ghalfi, Yehya Chakib & 1 & 1 \\
\hline Ghelich, Pezhman & 1 & 1 \\
\hline
\end{tabular}




\begin{tabular}{|c|c|c|}
\hline Author & Documents & Citations \\
\hline Golmohammadi, Mohammad Ghasem & 1 & 1 \\
\hline Gomez, Lorenzo Martinez & 1 & 1 \\
\hline Gonsalves, Dymphan F. & 1 & 1 \\
\hline Gouda, Shrouk Mohamed & 1 & 1 \\
\hline Govindharaj, Dhivyadharshini & 1 & 1 \\
\hline Guemra, K. & 1 & 1 \\
\hline Haghighatseir, Niloofar & 1 & 1 \\
\hline Hashmi, Farwa & 1 & 1 \\
\hline Hashmi, Ghulam Muhammad Mujtba & 1 & 1 \\
\hline Hassan, Hassan M. & 1 & 1 \\
\hline Hassan, Nazim & 1 & 1 \\
\hline Hegazy, Ehab & 1 & 1 \\
\hline Helal, Maher H. & 1 & 1 \\
\hline Hermawan, Bambang & 1 & 1 \\
\hline Hesaraki, Saeed & 1 & 1 \\
\hline Hoffmann, Ronaldo & 1 & 1 \\
\hline Hoghoghi, Atoosa & 1 & 1 \\
\hline Holban, Alina Maria & 1 & 1 \\
\hline Honarvar, Bizhan & 1 & 1 \\
\hline Honerio Franca, Adenilda Cristina & 1 & 1 \\
\hline Hooshdar, Pegah & 1 & 1 \\
\hline Hossain, K. M. Amjad & 1 & 1 \\
\hline Hosseinian Khatibi, Seyed Mahdi & 1 & 1 \\
\hline Hristoforou, Evangelos & 1 & 1 \\
\hline Hudyma, Arsen A. & 1 & 1 \\
\hline Hussain, Fida & 1 & 1 \\
\hline Hussain, Zainab & 1 & 1 \\
\hline Iacob, Mirela Maria & 1 & 1 \\
\hline Ibrahim, Mohamed Abbas & 1 & 1 \\
\hline Injorhor, Preeyaporn & 1 & 1 \\
\hline Ionescu, Ecaterina & 1 & 1 \\
\hline Ionescu, Petra & 1 & 1 \\
\hline Iordache, Florin & 1 & 1 \\
\hline Irmansyah, Irmansyah & 1 & 1 \\
\hline Ishak, Mohamad Ridzwan & 1 & 1 \\
\hline Ismail, Wan Norfazilah Wan & 1 & 1 \\
\hline Istrate, Claudia Mihaela & 1 & 1 \\
\hline Ivanushko, Yana G. & 1 & 1 \\
\hline Jacques, Rosangela Assis & 1 & 1 \\
\hline Jafarkhani, Mahboubeh & 1 & 1 \\
\hline Jagminas, Arunas & 1 & 1 \\
\hline Jahadi, Mahshid & 1 & 1 \\
\hline Jamiu, Adisa Muhammad & 1 & 1 \\
\hline Jan, Nida & 1 & 1 \\
\hline Janardan, S. & 1 & 1 \\
\hline Janardan, Sannapaneni & 1 & 1 \\
\hline Jancikova, Simona & 1 & 1 \\
\hline Jannat, Behrooz & 1 & 1 \\
\hline Javadi, Afshin & 1 & 1 \\
\hline Javadi, Mina & 1 & 1 \\
\hline Jivoinovici, Radita & 1 & 1 \\
\hline Joly, Nicolas & 1 & 1 \\
\hline Joseph, Lincy & 1 & 1 \\
\hline Joshi, Harish Chandra & 1 & 1 \\
\hline Ju, Zhiyu & 1 & 1 \\
\hline Kakh, Saeed Gharib Hosseini & 1 & 1 \\
\hline Kalantari, Ghazal & 1 & 1 \\
\hline Kambila, Vijaya Kumar & 1 & 1 \\
\hline Kamma, Sreenivasulu & 1 & 1 \\
\hline Karabanovas, Vitalijus & 1 & 1 \\
\hline Karki, Subhas S. & 1 & 1 \\
\hline
\end{tabular}




\begin{tabular}{|c|c|c|}
\hline Author & Documents & Citations \\
\hline Karpicz, Renata & 1 & 1 \\
\hline Kasipandian, Kasirajan & 1 & 1 \\
\hline Kazemzadeh, Asghar & 1 & 1 \\
\hline Kermanshahi, Rouha K. & 1 & 1 \\
\hline Kesarla, Mohan Kumar & 1 & 1 \\
\hline Khajehpour, Hossein & 1 & 1 \\
\hline Khakpour, Aboolfazal & 1 & 1 \\
\hline Khaleghi, Mahsa & 1 & 1 \\
\hline Khan, Aimal & 1 & 1 \\
\hline Khan, Amrita & 1 & 1 \\
\hline Khan, Hasmat & 1 & 1 \\
\hline Khanniri, Elham & 1 & 1 \\
\hline Khorasani, Mohammad Taghi & 1 & 1 \\
\hline Khorshahi, Hasan & 1 & 1 \\
\hline Khosravi, Kianoush & 1 & 1 \\
\hline Khurshid, Shazia & 1 & 1 \\
\hline Kim, Irina & 1 & 1 \\
\hline Kit, Ng Hou & 1 & 1 \\
\hline Klaic, Rodrigo & 1 & 1 \\
\hline Klein, Tais & 1 & 1 \\
\hline Komur, Ali Ihsan & 1 & 1 \\
\hline Koriem, Khaled Mohamed Mohamed & 1 & 1 \\
\hline Kothandan, Ram & 1 & 1 \\
\hline Kovalenko, Anatoliy E. & 1 & 1 \\
\hline Kravchuk, Larysa & 1 & 1 \\
\hline Kraysman, Natalia V. & 1 & 1 \\
\hline Kuanar, Bijayalaxmi & 1 & 1 \\
\hline Kuhn, Raquel C. & 1 & 1 \\
\hline Kulkarni, Sunil & 1 & 1 \\
\hline Kumari, Alka & 1 & 1 \\
\hline Kushnir, Marta, V & 1 & 1 \\
\hline Labbaci, Abdallah & 1 & 1 \\
\hline Laredj, Hocine & 1 & 1 \\
\hline Latifi, Ali Mohammad & 1 & 1 \\
\hline Leman, Zulkiflle & 1 & 1 \\
\hline Li, Zhongchun & 1 & 1 \\
\hline Lkrik, Abdelaziz & 1 & 1 \\
\hline Logoyda, Liliya & 1 & 1 \\
\hline Loos, Andrea & 1 & 1 \\
\hline Ludin, Norasikin Ahmad & 1 & 1 \\
\hline Lykov, Alexander & 1 & 1 \\
\hline Mabood, Fazal & 1 & 1 \\
\hline Machado, Izabela Trindade & 1 & 1 \\
\hline Mahapatra, Biswajita & 1 & 1 \\
\hline Mahmoud, Abdel Aziz & 1 & 1 \\
\hline Mahmoud, Sally M. & 1 & 1 \\
\hline Makegowda, Manjushree & 1 & 1 \\
\hline Malek, Wadeea & 1 & 1 \\
\hline Malik, Muhammad Azad & 1 & 1 \\
\hline Mallampalli, Bhanu & 1 & 1 \\
\hline Mamalis, Athanasios G. & 1 & 1 \\
\hline Manassero, Carlo & 1 & 1 \\
\hline Mansour, Mandi & 1 & 1 \\
\hline Mansouri, Seyed Soheil & 1 & 1 \\
\hline Marciniak, Cintia & 1 & 1 \\
\hline Maria, Kazi Hanium & 1 & 1 \\
\hline Martin, Patrick & 1 & 1 \\
\hline Martinez-Garcia, Marcos & 1 & 1 \\
\hline Martinez-Klimova, Elena & 1 & 1 \\
\hline Martins, Jordana Santos & 1 & 1 \\
\hline Massit, Asmaa & 1 & 1 \\
\hline
\end{tabular}




\begin{tabular}{|c|c|c|}
\hline Author & Documents & Citations \\
\hline Mehboob, Nida & 1 & 1 \\
\hline Mehrnejad, Faramarz & 1 & 1 \\
\hline Meikhail, Mahrous S. & 1 & 1 \\
\hline Memis, Elifcan & 1 & 1 \\
\hline Mendes, Luan Dos Santos & 1 & 1 \\
\hline Mihailescu, Patricia Elena & 1 & 1 \\
\hline Mikalauskaite, Agne & 1 & 1 \\
\hline Mikhailovich, Emelyanov Victor & 1 & 1 \\
\hline Mirzaei, Morteza & 1 & 1 \\
\hline Mishra, Sunanda & 1 & 1 \\
\hline Misri, Sairizal & 1 & 1 \\
\hline Mittal, Amit & 1 & 1 \\
\hline Mohamad, Faridah & 1 & 1 \\
\hline Mohamed, Eisawy R. & 1 & 1 \\
\hline Mohammed, Aya R. & 1 & 1 \\
\hline Mohareb, Rafat M. & 1 & 1 \\
\hline Mola, Adeleh & 1 & 1 \\
\hline Moradi, Rezvan Seid & 1 & 1 \\
\hline Morata, Antonio & 1 & 1 \\
\hline Moria, Hazim & 1 & 1 \\
\hline Mortazavian, Amir Mohammad & 1 & 1 \\
\hline Mousavi, Sayedali & 1 & 1 \\
\hline Mozafari, Mohammad R. & 1 & 1 \\
\hline Moztarzadeh, Fathollah & 1 & 1 \\
\hline Mukundaswamy, Chethan Kumar & 1 & 1 \\
\hline Muresan, Andrei & 1 & 1 \\
\hline Nachimuthu, Saraswathy & 1 & 1 \\
\hline Najm, Asmaa Soheil & 1 & 1 \\
\hline Nath, Deepak & 1 & 1 \\
\hline Nazemi, Adeleh & 1 & 1 \\
\hline Negandari, Ramin & 1 & 1 \\
\hline Nieto-Camacho, Antonio & 1 & 1 \\
\hline Nisar, Mohammad & 1 & 1 \\
\hline Nosotti, Maria Giulia & 1 & 1 \\
\hline Oksuz, Aysegul Uygun & 1 & 1 \\
\hline Olayinka, Akolade Jubril & 1 & 1 \\
\hline Ongun, Kerim & 1 & 1 \\
\hline Orlova, Natalia & 1 & 1 \\
\hline Ozaltun, Betul & 1 & 1 \\
\hline Padilla-Monroy, Salma & 1 & 1 \\
\hline Pakdel, Seyyed Mandi Vahid & 1 & 1 \\
\hline Pal, Abhisek & 1 & 1 \\
\hline Palani, R. & 1 & 1 \\
\hline Palanisamy, Kaliyan & 1 & 1 \\
\hline Panahi, Shariat & 1 & 1 \\
\hline Panda, Bibhu Prasad & 1 & 1 \\
\hline Pangan, Akbar Reformasi & 1 & 1 \\
\hline Parida, Siba Prasad & 1 & 1 \\
\hline Paroul, Natalia & 1 & 1 \\
\hline Pattammadath, Sajeesh & 1 & 1 \\
\hline Pattanaik, Archana & 1 & 1 \\
\hline Peleshok, Kateryna & 1 & 1 \\
\hline Pelinescu, Diana & 1 & 1 \\
\hline Pellizaro, Thales A. G. & 1 & 1 \\
\hline Perchyonok, V. Tamara & 1 & 1 \\
\hline Petchi, Iyappan & 1 & 1 \\
\hline Petzold, Gudrun & 1 & 1 \\
\hline Piatnochka, Volodymyr & 1 & 1 \\
\hline Piddubna, Antonina A. & 1 & 1 \\
\hline Pimentel, Tatiana Colombo & 1 & 1 \\
\hline Piponski, Marjan & 1 & 1 \\
\hline
\end{tabular}




\begin{tabular}{|c|c|c|}
\hline Author & Documents & Citations \\
\hline Pourjafar, Hadi & 1 & 1 \\
\hline Poveshchenko, Olga & 1 & 1 \\
\hline Pradhan, Abanti & 1 & 1 \\
\hline Pradhan, Debabrata & 1 & 1 \\
\hline Prajisya, P. T. & 1 & 1 \\
\hline Purohit, Soumya Ranjan & 1 & 1 \\
\hline Qureshi, Tahira & 1 & 1 \\
\hline Qureshi, Zahid Muhammad & 1 & 1 \\
\hline Rad, Aziz Homayouni & 1 & 1 \\
\hline Radu, Monica Violeta & 1 & 1 \\
\hline Rafiei, Pedram & 1 & 1 \\
\hline Rahman, Mohammad Azizur & 1 & 1 \\
\hline Rahmani, Sakineh & 1 & 1 \\
\hline Raiz, Nadia & 1 & 1 \\
\hline Rajamani, Lakshminarayanan & 1 & 1 \\
\hline Raji, P. & 1 & 1 \\
\hline Rakesh, Gunnam & 1 & 1 \\
\hline Ramakrishana, Seeram & 1 & 1 \\
\hline Ramirez-Apan, Teresa & 1 & 1 \\
\hline Ramteke, Pramod W. & 1 & 1 \\
\hline Rani, Aksha & 1 & 1 \\
\hline Ranjith, K. R. & 1 & 1 \\
\hline Rastegar, Mona & 1 & 1 \\
\hline Reato, Paloma Truccolo & 1 & 1 \\
\hline Reddy Bogireddy, Naveen Kumar & 1 & 1 \\
\hline Renitta, Emilin R. & 1 & 1 \\
\hline Reshef, Noam & 1 & 1 \\
\hline Rezzouk, Abdellah & 1 & 1 \\
\hline Ribeiro Barbosa, Maria Paula & 1 & 1 \\
\hline Rodrigues, Eliseu & 1 & 1 \\
\hline Rotomskis, Ricardas & 1 & 1 \\
\hline Rout, Prasant Kumar & 1 & 1 \\
\hline Ruksakulpiwat, Chaiwat & 1 & 1 \\
\hline Ruksakulpiwat, Yupaporn & 1 & 1 \\
\hline Rusu, Elena & 1 & 1 \\
\hline Safenkova, Irina, V & 1 & 1 \\
\hline Saha, Dilip Kumar & 1 & 1 \\
\hline Sahithya, Chamarthy Sai & 1 & 1 \\
\hline Sahli, Fatima Zohra & 1 & 1 \\
\hline Saidi, Mohamed & 1 & 1 \\
\hline Saidi-Amroun, Nadia & 1 & 1 \\
\hline Salehi, Zeinab & 1 & 1 \\
\hline Salem-Bekhit, Mounir M. & 1 & 1 \\
\hline Salem-Bekhit, Mounir Mohamed & 1 & 1 \\
\hline Saliba, Lucas Falquetti & 1 & 1 \\
\hline Salit, Mohd Sapuan & 1 & 1 \\
\hline Sallet, Daniela & 1 & 1 \\
\hline Samara, Athina & 1 & 1 \\
\hline Sani, Hakimeh Moghaddas & 1 & 1 \\
\hline Santamaria-Aguirre, Javier & 1 & 1 \\
\hline Saorin Puton, Bruna Maria & 1 & 1 \\
\hline Sarghie, Larisa & 1 & 1 \\
\hline Sari, Ajeng Arum & 1 & 1 \\
\hline Sariri, Reyhaneh & 1 & 1 \\
\hline Sarma, M. S. S. R. K. N. & 1 & 1 \\
\hline Sarmadi, Bahareh & 1 & 1 \\
\hline Sassi, Mohamed & 1 & 1 \\
\hline Schwarz, Simona & 1 & 1 \\
\hline Sedaghat, Mahshid & 1 & 1 \\
\hline Selvarasi, J. & 1 & 1 \\
\hline Sen, Ismail & 1 & 1 \\
\hline
\end{tabular}




\begin{tabular}{|c|c|c|}
\hline Author & Documents & Citations \\
\hline Sepahvandi, Azadeh & 1 & 1 \\
\hline Sequetto, Priscila L. & 1 & 1 \\
\hline Serra, Osvaldo Antonio & 1 & 1 \\
\hline Sevindik, Mustafa & 1 & 1 \\
\hline Sezer, Gokhan & 1 & 1 \\
\hline Shah, Syed Sadaqat & 1 & 1 \\
\hline Shahbazi, Ali & 1 & 1 \\
\hline Shahi, Navin Chandra & 1 & 1 \\
\hline Shaikhiev, Ildar G. & 1 & 1 \\
\hline Sharma, Apoorva & 1 & 1 \\
\hline Sharma, Vasudha & 1 & 1 \\
\hline Sheibani, Hassan & 1 & 1 \\
\hline Sher, Muhammad & 1 & 1 \\
\hline Shevchenko, Stanislav, I & 1 & 1 \\
\hline Shidlovskyi, Viktor O. & 1 & 1 \\
\hline Shiprath, Kudelallu & 1 & 1 \\
\hline Singh, Saurabh & 1 & 1 \\
\hline Singh, Smita & 1 & 1 \\
\hline Skrypska, Olga, V & 1 & 1 \\
\hline Snoeck, Robert & 1 & 1 \\
\hline Sona, K. P. & 1 & 1 \\
\hline Souidi, Kaies & 1 & 1 \\
\hline Spannemberg, Stefani S. & 1 & 1 \\
\hline Spesivtseva, Svetlana E. & 1 & 1 \\
\hline Sruthi, Durga P. & 1 & 1 \\
\hline Stanislav, Alexandra Alina & 1 & 1 \\
\hline Stanislav, Alina Alexandra & 1 & 1 \\
\hline Steinbach, Christine & 1 & 1 \\
\hline Stephen, Adeyemi Oluyomi & 1 & 1 \\
\hline Stoica, Ileana & 1 & 1 \\
\hline Suarez-Lepe, Jose A. & 1 & 1 \\
\hline $\begin{array}{ll}\text { Subramanian, } & \text { Vadakkeputhanmadom } \\
\text { Krishnaiyer } & \end{array}$ & 1 & 1 \\
\hline Subramanian, Vasuki & 1 & 1 \\
\hline Suciu, Ileana & 1 & 1 \\
\hline Suciu, Ioana & 1 & 1 \\
\hline Sukardan, Danny & 1 & 1 \\
\hline Sukumaran, Sandya & 1 & 1 \\
\hline Sullca Grimaldez, Lourdes & 1 & 1 \\
\hline Sundalian, Melvia & 1 & 1 \\
\hline Suresh, Kothanlasu & 1 & 1 \\
\hline Surovtseva, Maria & 1 & 1 \\
\hline Sverguzova, Svetlana V. & 1 & 1 \\
\hline Swamy, Budde Kumar & 1 & 1 \\
\hline Swamy, Budde Kumara & 1 & 1 \\
\hline Sydorchuk, Larysa P. & 1 & 1 \\
\hline Taghdir, Majid & 1 & 1 \\
\hline Taheri, Mansoor & 1 & 1 \\
\hline Taskaeva, Iuliia & 1 & 1 \\
\hline Tatmatsu Rocha, Jose Carlos & 1 & 1 \\
\hline Thirumurugan, R. & 1 & 1 \\
\hline Thota, Sreekanth & 1 & 1 \\
\hline Tkach, Volodymyr V. & 1 & 1 \\
\hline Tkachuk, Nina P. & 1 & 1 \\
\hline Toba, Anifowoshe Abass & 1 & 1 \\
\hline Tolaba, Angel G. & 1 & 1 \\
\hline Tondepu, Subbaiah & 1 & 1 \\
\hline Topcu, Aykut & 1 & 1 \\
\hline Tounsi, Moufida Saidani & 1 & 1 \\
\hline Tremlova, Bohuslava & 1 & 1 \\
\hline Tucceri, Ricardo & 1 & 1 \\
\hline
\end{tabular}




\begin{tabular}{|c|c|c|}
\hline Author & Documents & Citations \\
\hline Tyagi, Nidhi & 1 & 1 \\
\hline Urnau, Leticia & 1 & 1 \\
\hline Vasilevich, Natalya I. & 1 & 1 \\
\hline Veras, Mirella & 1 & 1 \\
\hline Verma, Santosh Kumar & 1 & 1 \\
\hline Verveha, Bohdana M. & 1 & 1 \\
\hline Viktorovich, Emelyanov Viktor & 1 & 1 \\
\hline Vilela, Daisy De Araujo & 1 & 1 \\
\hline Wadood, Syed Fazal & 1 & 1 \\
\hline Wali, Hazrat & 1 & 1 \\
\hline Wannes, Wissem Aidi & 1 & 1 \\
\hline Wu, Changzeng & 1 & 1 \\
\hline Wu, Xingxing & 1 & 1 \\
\hline Wu, Zucheng & 1 & 1 \\
\hline $\mathrm{Xu}, \mathrm{Jie}$ & 1 & 1 \\
\hline $\mathrm{Xu}, \mathrm{Wei}$ & 1 & 1 \\
\hline $\mathrm{Xu}$, Zhihong & 1 & 1 \\
\hline Yaghmaei, Parichehreh & 1 & 1 \\
\hline Yagodynets, Petro, I & 1 & 1 \\
\hline Yang, $\mathrm{Xu}$ & 1 & 1 \\
\hline Yanuar, Arry & 1 & 1 \\
\hline Yarothckina, Angela N. & 1 & 1 \\
\hline Yaseen, Mifftha & 1 & 1 \\
\hline Yoong, Wong Sim & 1 & 1 \\
\hline Yu, Binbin & 1 & 1 \\
\hline Yuniarto, Adhi & 1 & 1 \\
\hline Zakharchuk, Uliana & 1 & 1 \\
\hline Zandu, Simran Kaur & 1 & 1 \\
\hline Zavgorodnyi, Serhiy M. & 1 & 1 \\
\hline Zhang, Shengmiao & 1 & 1 \\
\hline Zheng, Haoyue & 1 & 1 \\
\hline Zhu, Xiaoshan & 1 & 1 \\
\hline Maria Virginia, Mateu-Armad & 5 & 0 \\
\hline Abelardo, Camacho-Luis & 4 & 0 \\
\hline Alejandara, Garcimarrero E. & 4 & 0 \\
\hline Ditu, Lia-Mara & 4 & 0 \\
\hline Fouda, Abd El-Aziz S. & 3 & 0 \\
\hline Kvan, Olga & 3 & 0 \\
\hline Nanda, Binita & 3 & 0 \\
\hline Nanda, Braja B. & 3 & 0 \\
\hline Abdullah, Faizuan & 2 & 0 \\
\hline Abdullah, Hasan Zuhudi & 2 & 0 \\
\hline Aebisher, David & 2 & 0 \\
\hline Alexandru, Ionela & 2 & 0 \\
\hline Anagnostopoulos, Constantine & 2 & 0 \\
\hline Andreescu, Nicoleta & 2 & 0 \\
\hline Asadi-Shekaari, Majid & 2 & 0 \\
\hline Bakri, Youssef & 2 & 0 \\
\hline Bartusik, Dorota & 2 & 0 \\
\hline Bouyahya, Abdelhakim & 2 & 0 \\
\hline Carrera Sanchez, Cecilio & 2 & 0 \\
\hline Chadha, Hina & 2 & 0 \\
\hline Cianciolo, Simona & 2 & 0 \\
\hline Constantinescu, Ileana & 2 & 0 \\
\hline Da Costa, Liovando Marciano & 2 & 0 \\
\hline Da Costa, Marcelo Rocha & 2 & 0 \\
\hline Dash, Upendra Nath & 2 & 0 \\
\hline De Oliveira, Tania Toledo & 2 & 0 \\
\hline Dehghani, Leila & 2 & 0 \\
\hline Dong, Xiaofeng & 2 & 0 \\
\hline Dulamea, Octaviana Adriana & 2 & 0 \\
\hline
\end{tabular}




\begin{tabular}{|c|c|c|}
\hline Author & Documents & Citations \\
\hline Duskaev, Galimzhan & 2 & 0 \\
\hline Elias, Rita S. & 2 & 0 \\
\hline Esmaeilpour, Khadijeh & 2 & 0 \\
\hline Ghita, Gina & 2 & 0 \\
\hline Grumezescu, Alexandru Mihai & 2 & 0 \\
\hline Haleem, Eslam Abdel & 2 & 0 \\
\hline Kalantaripour, Taj Pari & 2 & 0 \\
\hline Kenawy, Sayed H. & 2 & 0 \\
\hline Kong, Ling Bing & 2 & 0 \\
\hline Kosyan, Dianna & 2 & 0 \\
\hline Kumar, Arvind & 2 & 0 \\
\hline Laslo, Lucian & 2 & 0 \\
\hline Li, Shaobo & 2 & 0 \\
\hline Li, Xiuying & 2 & 0 \\
\hline Luo, Wenyan & 2 & 0 \\
\hline Mathiyalagan, Siva & 2 & 0 \\
\hline Mezhueva, Larisa & 2 & 0 \\
\hline Munir, Shahzad & 2 & 0 \\
\hline Nandi, Shoma Paul & 2 & 0 \\
\hline Niculescu, Marius & 2 & 0 \\
\hline Parfenova, Aksana M. & 2 & 0 \\
\hline Pereira, Wander Lopes & 2 & 0 \\
\hline Pervez, Rashid & 2 & 0 \\
\hline Prastyo, Dedy Dwi & 2 & 0 \\
\hline Puiu, Maria & 2 & 0 \\
\hline Rashidiani, Jamal & 2 & 0 \\
\hline Rasit, Nazaitulshila & 2 & 0 \\
\hline Sahoo, Naresh Kumar & 2 & 0 \\
\hline Shaikh, Tanveer Mahamad Alli & 2 & 0 \\
\hline Sharma, Angkita & 2 & 0 \\
\hline Singh, Pooja & 2 & 0 \\
\hline Tomas, Lopez-Gutierrez & 2 & 0 \\
\hline Udriste, Anca Amalia & 2 & 0 \\
\hline Virginia, Mateu-Armad & 2 & 0 \\
\hline Wang, Yonghong & 2 & 0 \\
\hline Wang, Yu & 2 & 0 \\
\hline Xiao, Zhuohao & 2 & 0 \\
\hline Xu, Xiaoqiang & 2 & 0 \\
\hline Yang, Jie & 2 & 0 \\
\hline Zabot, Giovani Leone & 2 & 0 \\
\hline Zare, Reza & 2 & 0 \\
\hline Zhang, Xia & 2 & 0 \\
\hline Abdel-Rahim, Emam A. & 1 & 0 \\
\hline Abdelghany, Amr & 1 & 0 \\
\hline Abdelmalek, Ilham & 1 & 0 \\
\hline Abdelmoaty, Alaa Salah & 1 & 0 \\
\hline Abedi, Farshid & 1 & 0 \\
\hline Abolhassanzadeh, Zohreh & 1 & 0 \\
\hline Abou Gabal, Raghda & 1 & 0 \\
\hline Abou-Okeil, Ashraf & 1 & 0 \\
\hline About-Jaudet, Elie & 1 & 0 \\
\hline Abutorabi, Roshanak & 1 & 0 \\
\hline Achille, Tarsitano & 1 & 0 \\
\hline Adil, Muhammad & 1 & 0 \\
\hline Adjeng, Andi Nafisah Tendri & 1 & 0 \\
\hline Adriano, Piattelli & 1 & 0 \\
\hline Afonin, Andrey N. & 1 & 0 \\
\hline Afsharian, Zahra & 1 & 0 \\
\hline Afyouni, Nazgol Esmalian & 1 & 0 \\
\hline Aggouf, Moufida & 1 & 0 \\
\hline Aghajani, Zahra & 1 & 0 \\
\hline
\end{tabular}




\begin{tabular}{|c|c|c|}
\hline Author & Documents & Citations \\
\hline Aghamohammadi, Mohammad & 1 & 0 \\
\hline Aghazadeh, Marziyeh & 1 & 0 \\
\hline Aghazadeh, Zahra & 1 & 0 \\
\hline Agustriawan, David & 1 & 0 \\
\hline Ahangari, Zohreh & 1 & 0 \\
\hline Ahmad, Sajjad & 1 & 0 \\
\hline Ahmad, Shakeel & 1 & 0 \\
\hline Ahmadov, Ismat S. & 1 & 0 \\
\hline Ahmadov, Ismat Suleyman & 1 & 0 \\
\hline Ahmadpour, Anahita & 1 & 0 \\
\hline Ahmed, Basem S. & 1 & 0 \\
\hline Ahmed, Iftikhar & 1 & 0 \\
\hline Ahmed, Irfan & 1 & 0 \\
\hline Ahmed, Muniruddin & 1 & 0 \\
\hline Ahuja, Munish & 1 & 0 \\
\hline Ai, Jafar & 1 & 0 \\
\hline Aissaoui, Amel & 1 & 0 \\
\hline Akhtar, Mohammad Saleem & 1 & 0 \\
\hline Akselevich, Vasilisa & 1 & 0 \\
\hline Aktumsek, Abdurrahman & 1 & 0 \\
\hline Al Abdullah, Wahib & 1 & 0 \\
\hline Al-Ahmed, Zehba A. & 1 & 0 \\
\hline Al-Anssari, Reham Adnan & 1 & 0 \\
\hline Al-Masoudi, Najim A. & 1 & 0 \\
\hline Al-Namnam, Nisreen Mohammed & 1 & 0 \\
\hline Alajmi, Assil Najeeb & 1 & 0 \\
\hline Alatas, Husin & 1 & 0 \\
\hline Aleksey, Ruchay & 1 & 0 \\
\hline Aleksey, Sizentsov N. & 1 & 0 \\
\hline Aleksey, Torshkov A. & 1 & 0 \\
\hline Aleshkov, Alexey & 1 & 0 \\
\hline Alessandro, Spadari & 1 & 0 \\
\hline Alexandrea, Marin-Parra & 1 & 0 \\
\hline Alexeevich, Belov Alexey & 1 & 0 \\
\hline Alexeevna, Vaniushenkova Anna & 1 & 0 \\
\hline Aleynikov, Andrey Yu. & 1 & 0 \\
\hline Alfonso-Jimenez, Alondra & 1 & 0 \\
\hline Alghuthaymi, Mousa & 1 & 0 \\
\hline Alhiti, Hazim Abdul Rahman & 1 & 0 \\
\hline Ali, Umar & 1 & 0 \\
\hline Aljadaan, Shaker A. N. & 1 & 0 \\
\hline Aljuhani, Enas & 1 & 0 \\
\hline Almeida, Thiago & 1 & 0 \\
\hline Altan, Halenur & 1 & 0 \\
\hline Aminizadeh, Soheil & 1 & 0 \\
\hline Ammar, Hussein & 1 & 0 \\
\hline Ammare, Yibrah & 1 & 0 \\
\hline Amr, Ahmed & 1 & 0 \\
\hline Andrea, Valsesia & 1 & 0 \\
\hline Andriets, Volodymyr V. & 1 & 0 \\
\hline Anissa, Belhadj & 1 & 0 \\
\hline Anna, Popova N. & 1 & 0 \\
\hline Annapaola, Parrilli & 1 & 0 \\
\hline Aparicio, Rosa & 1 & 0 \\
\hline Aquino, Lidia Raquel C. & 1 & 0 \\
\hline Arif, Sekh Md & 1 & 0 \\
\hline Arifin, Muhammad Zainul & 1 & 0 \\
\hline Arsenovych, Hudyma Arsen & 1 & 0 \\
\hline Arslaner, Cennet & 1 & 0 \\
\hline Arslanova, Lenara & 1 & 0 \\
\hline Artem, Bykov & 1 & 0 \\
\hline
\end{tabular}




\begin{tabular}{|c|c|c|}
\hline Author & Documents & Citations \\
\hline Artem, Bykov, V & 1 & 0 \\
\hline Asare, Ebenezer Aquisman & 1 & 0 \\
\hline Aslam, Asim & 1 & 0 \\
\hline Asnaoui, Hassan & 1 & 0 \\
\hline Atabati, Elham & 1 & 0 \\
\hline Ataei, Abdollah & 1 & 0 \\
\hline Atanasova, Ventzeslava & 1 & 0 \\
\hline Atici, Emine & 1 & 0 \\
\hline Avola, Roberto & 1 & 0 \\
\hline Axelevitch, Alexander & 1 & 0 \\
\hline Ayad, Mohamed N. & 1 & 0 \\
\hline Ayar, Ahmet & 1 & 0 \\
\hline Ayoubi-Chianeh, Mojgan & 1 & 0 \\
\hline Azami, Mahmoud & 1 & 0 \\
\hline Azelee, Ihsan Wan & 1 & 0 \\
\hline Azimi, Alireza & 1 & 0 \\
\hline Azimi-Nezhad, Mohsen & 1 & 0 \\
\hline Azmi, Alyza Azzura Abd Rahman & 1 & 0 \\
\hline Azovtseva, Natalya A. & 1 & 0 \\
\hline B-Rao, Chandrika & 1 & 0 \\
\hline Babu, B. Sridhar & 1 & 0 \\
\hline Badr, Gamila El-Sayed Abdalla & 1 & 0 \\
\hline Badra, Haddou & 1 & 0 \\
\hline Bagheri, Amir & 1 & 0 \\
\hline Bagmut, Irina & 1 & 0 \\
\hline Bahmaei, Manochehr & 1 & 0 \\
\hline Bahrami, Reza & 1 & 0 \\
\hline Bahri, Parisima Haddad & 1 & 0 \\
\hline Bajaj, Komal & 1 & 0 \\
\hline Bakhshpour, Monireh & 1 & 0 \\
\hline Bakil, Siti Natrah Abdul & 1 & 0 \\
\hline Bakshaliyeva, Konul F. & 1 & 0 \\
\hline Balahbib, Abdelaali & 1 & 0 \\
\hline Baloch, Zulqarnain & 1 & 0 \\
\hline Bansod, Ankur, V & 1 & 0 \\
\hline Barabadi, Zahra & 1 & 0 \\
\hline Baranovych, Diana & 1 & 0 \\
\hline Barbara, Dozza & 1 & 0 \\
\hline Barrientos, Kaory & 1 & 0 \\
\hline Bartzeliotis, Antonios & 1 & 0 \\
\hline Barysheva, Elena & 1 & 0 \\
\hline Bashiri, Hamideh & 1 & 0 \\
\hline Bedi, Pooja & 1 & 0 \\
\hline Behera, Manoranjan & 1 & 0 \\
\hline Beklemishev, Mikhail K. & 1 & 0 \\
\hline Bengharez, Zohra & 1 & 0 \\
\hline Bernal-Ballen, Andres & 1 & 0 \\
\hline Bety, Sarabia-Alcocer & 1 & 0 \\
\hline Beydokhti, Hossein & 1 & 0 \\
\hline Bhadraiah, Umesha K. & 1 & 0 \\
\hline Bhatt, Anjani K. & 1 & 0 \\
\hline Bhattacharya, Shramana & 1 & 0 \\
\hline Bhattarai, Ajaya & 1 & 0 \\
\hline Bidjou-Haiour, Chahra & 1 & 0 \\
\hline Bilgin, Bilal & 1 & 0 \\
\hline Biswal, Susanta Kumar & 1 & 0 \\
\hline Bocharov, Andriy, V & 1 & 0 \\
\hline Bohdanivna, Hetmaniuk Iryna & 1 & 0 \\
\hline Boldyrev, Artur & 1 & 0 \\
\hline Bonartsev, Anton & 1 & 0 \\
\hline Bonartseva, Garina & 1 & 0 \\
\hline
\end{tabular}




\begin{tabular}{|c|c|c|}
\hline Author & Documents & Citations \\
\hline Bordoloi, Manobjyoti & 1 & 0 \\
\hline Borges, Franz Viana & 1 & 0 \\
\hline Bouariu, Alexandra & 1 & 0 \\
\hline Boudali, Lilia Khalfallah & 1 & 0 \\
\hline Brahmbhatt, Harshad & 1 & 0 \\
\hline Brown, John W. & 1 & 0 \\
\hline Brun, Thiarles & 1 & 0 \\
\hline Buitrago, Laura & 1 & 0 \\
\hline Burik, Mikhail & 1 & 0 \\
\hline Bykov, Atrem & 1 & 0 \\
\hline Byrappa, K. & 1 & 0 \\
\hline Byrappa, Satish K. & 1 & 0 \\
\hline Cabrera-Tuz, Jhair & 1 & 0 \\
\hline Camacho-Luis, Abelardo & 1 & 0 \\
\hline Carbone, Claudia & 1 & 0 \\
\hline Cardea, Stefano & 1 & 0 \\
\hline Ceni, Gustavo & 1 & 0 \\
\hline Ceylan, Kevser Betul & 1 & 0 \\
\hline Chaitra, Turuvekere Krishnamurthy & 1 & 0 \\
\hline Chandra & 1 & 0 \\
\hline Chang, Su-Youne & 1 & 0 \\
\hline Chee, Ching Yern & 1 & 0 \\
\hline Chelariu, Mihaela & 1 & 0 \\
\hline Chethan, Javarasetty & 1 & 0 \\
\hline Chin, Suk Fun & 1 & 0 \\
\hline Chitsaz, Niloofar & 1 & 0 \\
\hline Chua, Lee Suan & 1 & 0 \\
\hline Chuan, Lee Te & 1 & 0 \\
\hline Cimpoeru, Cristina & 1 & 0 \\
\hline Ciobotaru, Irina-Elena & 1 & 0 \\
\hline Columbus, Soumya & 1 & 0 \\
\hline Confortin, Tassia & 1 & 0 \\
\hline Constantinescu, Vlad & 1 & 0 \\
\hline Corsaro, Roberta & 1 & 0 \\
\hline Cotar, Ani Ioana & 1 & 0 \\
\hline Crespi, Francesco & 1 & 0 \\
\hline Cristea, Violeta-Corina & 1 & 0 \\
\hline Cruciani, Giuseppe & 1 & 0 \\
\hline Csoka, Levente & 1 & 0 \\
\hline Curkovic, Lidija & 1 & 0 \\
\hline Curtis, Robin & 1 & 0 \\
\hline Dabaghi, Parviz & 1 & 0 \\
\hline Dahman, Yaser & 1 & 0 \\
\hline Daniel Jr, Jair Joao & 1 & 0 \\
\hline Dantas Neto, Afonso A. & 1 & 0 \\
\hline Daoudi, Kais & 1 & 0 \\
\hline Das, Arun Kumar & 1 & 0 \\
\hline Das, Dorothy & 1 & 0 \\
\hline Das, Pundarikaksha & 1 & 0 \\
\hline De Barros Neto, Eduardo L. & 1 & 0 \\
\hline De Castro Dantas, Tereza N. & 1 & 0 \\
\hline De Marco, Iolanda & 1 & 0 \\
\hline Dehuri, Sujit K. & 1 & 0 \\
\hline Dehury, Sujit K. & 1 & 0 \\
\hline Dekamin, Mohammad G. & 1 & 0 \\
\hline Delahay, Gerard & 1 & 0 \\
\hline Demaman Oro, Carolina E. & 1 & 0 \\
\hline Demirci, Salih Cercis & 1 & 0 \\
\hline Denev, Panteley & 1 & 0 \\
\hline Derakhshandeh, Maryam & 1 & 0 \\
\hline Devi, Niharbala & 1 & 0 \\
\hline
\end{tabular}




\begin{tabular}{|c|c|c|}
\hline Author & Documents & Citations \\
\hline Devi, Savita & 1 & 0 \\
\hline Diacu, Elena & 1 & 0 \\
\hline Dianna, Kosyan & 1 & 0 \\
\hline Diaz Cedillo, Francisco & 1 & 0 \\
\hline Dikheel, Tahir Rissan & 1 & 0 \\
\hline Diwan, Prakash V. & 1 & 0 \\
\hline Djauhari, Arlin Besari & 1 & 0 \\
\hline Doddramappa, Shridevi D. & 1 & 0 \\
\hline Dong Zhaoxin & 1 & 0 \\
\hline Doostmohammadi, Razieh & 1 & 0 \\
\hline Dos Santos, Maicon S. N. & 1 & 0 \\
\hline Dragu, Denisa & 1 & 0 \\
\hline Driva, Paraskevi & 1 & 0 \\
\hline Droepenu, Eric Kwabena & 1 & 0 \\
\hline Drummen, Gregor P. C. & 1 & 0 \\
\hline Duan, Xiaohui & 1 & 0 \\
\hline Dubrovskis, Vilis & 1 & 0 \\
\hline Dudhe, Anshu & 1 & 0 \\
\hline Dudko, Andrey & 1 & 0 \\
\hline Duhan, Neha & 1 & 0 \\
\hline Dzhavakhiya, Vitaly & 1 & 0 \\
\hline Dzhuryak, Valentina & 1 & 0 \\
\hline Echeverri, Juan D. & 1 & 0 \\
\hline Egodage, Shantha & 1 & 0 \\
\hline El Hamdaoui, Lahcen & 1 & 0 \\
\hline El Magdoub, Hekmat M. & 1 & 0 \\
\hline El Moussaouiti, Mohammed & 1 & 0 \\
\hline El Omari, Nasreddine & 1 & 0 \\
\hline El-Hennawi, Heba & 1 & 0 \\
\hline El-Sherbieny, Sherien M. R. & 1 & 0 \\
\hline Elena, Rusakova & 1 & 0 \\
\hline Eli Alejandra, Garcimarrero-Espino & 1 & 0 \\
\hline Elshemy, Nagla & 1 & 0 \\
\hline Esfandiari, Ebrahim & 1 & 0 \\
\hline Estrella-Barron, Raquel & 1 & 0 \\
\hline Evgeniy, Kagakin I. & 1 & 0 \\
\hline Ezoji, Fariba & 1 & 0 \\
\hline Ezzat, Dina & 1 & 0 \\
\hline Faghri, Mohammad & 1 & 0 \\
\hline Faghri, Mohammed & 1 & 0 \\
\hline Faisal, Shah & 1 & 0 \\
\hline Fallah, Niloofar & 1 & 0 \\
\hline Falsafi, Parisa & 1 & 0 \\
\hline Fanuli, Matteo & 1 & 0 \\
\hline Faramarzpour, Mahsa & 1 & 0 \\
\hline Farhana, Nikhat & 1 & 0 \\
\hline Fauziyah, Nur & 1 & 0 \\
\hline Felcman, Judith & 1 & 0 \\
\hline Ferdous, Kazi Sayma & 1 & 0 \\
\hline Figueroa-Valverde, Lauro & 1 & 0 \\
\hline Filardi, Marcelo Augusto & 1 & 0 \\
\hline Firmansyah, Adang & 1 & 0 \\
\hline Foot, Peter J. S. & 1 & 0 \\
\hline Francescut, Lorenza & 1 & 0 \\
\hline Fung, Hau Zhe & 1 & 0 \\
\hline Furmuly, Mubasher & 1 & 0 \\
\hline Gaidi, Mounir & 1 & 0 \\
\hline Garcia-Cervera, Elodia & 1 & 0 \\
\hline Gargari, Seyed Latif Mousavi & 1 & 0 \\
\hline Gasimov, Eldar K. & 1 & 0 \\
\hline Gerasimov, Alexander & 1 & 0 \\
\hline
\end{tabular}




\begin{tabular}{|c|c|c|}
\hline Author & Documents & Citations \\
\hline Gharehaghaji, Nahideh & 1 & 0 \\
\hline Ghaztar, Muhammad Mustakim Mohd & 1 & 0 \\
\hline Ghedini, Elena & 1 & 0 \\
\hline Ghervan, Marian & 1 & 0 \\
\hline Gholian, Mohammad Mandi & 1 & 0 \\
\hline Ghorab, Mahmoud & 1 & 0 \\
\hline Ghosh, Samaresh & 1 & 0 \\
\hline Ghosh, Usha & 1 & 0 \\
\hline Giallongo, Cesarina & 1 & 0 \\
\hline Giannakos, Alexander & 1 & 0 \\
\hline Giorgio, Lesci Isidoro & 1 & 0 \\
\hline Giovanna, Iezzi & 1 & 0 \\
\hline Giri, Pankaj & 1 & 0 \\
\hline Gladishev, Andrey R. & 1 & 0 \\
\hline Gladisheva, Anastasiya V. & 1 & 0 \\
\hline Golfeshan, Farzaneh & 1 & 0 \\
\hline Golizadeh, Mortaza & 1 & 0 \\
\hline Golshani, Moazameh & 1 & 0 \\
\hline Gomes Martins, Carlos Henrique & 1 & 0 \\
\hline Gracy, Aroon & 1 & 0 \\
\hline Gradisteanu, Gratiela & 1 & 0 \\
\hline Grosu, Mirela & 1 & 0 \\
\hline Grynchuk, Andriy F. & 1 & 0 \\
\hline Grynchuk, Fedir, V & 1 & 0 \\
\hline Guastaldi, Antonio Carlos & 1 & 0 \\
\hline Guerrero-Escalante, Sebastian & 1 & 0 \\
\hline Guma, Manaf A. & 1 & 0 \\
\hline Gunaseelan, Rajeswari & 1 & 0 \\
\hline Gunay, Mehmet & 1 & 0 \\
\hline Guo, Xingjia & 1 & 0 \\
\hline Guzzetti, Karina A. & 1 & 0 \\
\hline Hadi, Saeid & 1 & 0 \\
\hline Hadjizadeh, Afra & 1 & 0 \\
\hline Haggage, Karima & 1 & 0 \\
\hline Haghighatnia, Yaghoub & 1 & 0 \\
\hline Hairom, Nur Hanis Hayati & 1 & 0 \\
\hline Hakeim, Osama A. & 1 & 0 \\
\hline Hamad, Mohamed N. F. & 1 & 0 \\
\hline Hamedi, Azadeh & 1 & 0 \\
\hline Hamidi, Mehrdad & 1 & 0 \\
\hline Hammam, Mohamed M. & 1 & 0 \\
\hline Han, Chengliang & 1 & 0 \\
\hline Hanna, Adly Abdalla & 1 & 0 \\
\hline Hao, Aijun & 1 & 0 \\
\hline Harbar, Oleksandr & 1 & 0 \\
\hline Hartati, Fadjar Kurnia & 1 & 0 \\
\hline Hashemibeni, Batool & 1 & 0 \\
\hline Hassan, Amir & 1 & 0 \\
\hline Hassanzadeh-Tabrizi, S. A. & 1 & 0 \\
\hline Hau-Heredia, Lenin & 1 & 0 \\
\hline Hau-Heredia-Lenin & 1 & 0 \\
\hline Hayat, Rifat & 1 & 0 \\
\hline Herrera-Meza, Socorro & 1 & 0 \\
\hline Horishniy, Ihor M. & 1 & 0 \\
\hline Hossam, Sameh & 1 & 0 \\
\hline Hosseini, Nikoo & 1 & 0 \\
\hline Hosseini, Sorour & 1 & 0 \\
\hline Hosseinvand, Amir & 1 & 0 \\
\hline Hozeifi, Samane & 1 & 0 \\
\hline Huang, Ziyue & 1 & 0 \\
\hline Hussain, Mubashir & 1 & 0 \\
\hline
\end{tabular}




\begin{tabular}{|c|c|c|}
\hline Author & Documents & Citations \\
\hline Hussain, Munir & 1 & 0 \\
\hline Hussain, Sajjad & 1 & 0 \\
\hline Iacob, Maria Mirela & 1 & 0 \\
\hline Ibrahim, Medhtat A. & 1 & 0 \\
\hline Ibrahim, Nik Noor Idayu Nik & 1 & 0 \\
\hline Ibrahim, Slamet & 1 & 0 \\
\hline Idrees, Muhammad & 1 & 0 \\
\hline Idris, Maizlinda & 1 & 0 \\
\hline Idris, Maizlinda Izwana & 1 & 0 \\
\hline Iftoda, Oksana & 1 & 0 \\
\hline Ihorivna, Hanberher Iryna & 1 & 0 \\
\hline Ihsan, Sunandar & 1 & 0 \\
\hline Ijsselstijn, Linda & 1 & 0 \\
\hline Imran, Muhammad Saeed & 1 & 0 \\
\hline Iqbal, M. Zafar & 1 & 0 \\
\hline Iramain, Maximiliano A. & 1 & 0 \\
\hline Ismail, Arashidatul Akmar & 1 & 0 \\
\hline Ismail, Razali & 1 & 0 \\
\hline Ivashchuk, Serhiy & 1 & 0 \\
\hline Ivashkin, Mikhail & 1 & 0 \\
\hline Ivchenko, Vladimir Aleksandrovich & 1 & 0 \\
\hline Iyappan, P. & 1 & 0 \\
\hline Jabrailzade, Sabia M. & 1 & 0 \\
\hline Jafari, Ayyoob & 1 & 0 \\
\hline Jafari, Sanaz & 1 & 0 \\
\hline Jahromi, Mohammad Ali Farboodniay & 1 & 0 \\
\hline Jain, Seema Mahor & 1 & 0 \\
\hline Jalal, Abdullah & 1 & 0 \\
\hline Jalali, Atefeh & 1 & 0 \\
\hline Jamal, Muhammad Ameen & 1 & 0 \\
\hline Jamil, Arslan & 1 & 0 \\
\hline Jamshidian, Saeid & 1 & 0 \\
\hline Jana, Batakrishna & 1 & 0 \\
\hline Jana, Snehasis & 1 & 0 \\
\hline Janardhan, S. & 1 & 0 \\
\hline Janghorban, Sadaf & 1 & 0 \\
\hline Jaramillo, Marisol & 1 & 0 \\
\hline Jattak, Zafarullah & 1 & 0 \\
\hline Javed, Nasir & 1 & 0 \\
\hline Jayakodi, Santhoshkumar & 1 & 0 \\
\hline Jayash, Soher Nagi & 1 & 0 \\
\hline Jin Yi & 1 & 0 \\
\hline Jorobekova, Sharipa & 1 & 0 \\
\hline Josefa, Pat-Estrella & 1 & 0 \\
\hline Kacar, Dogan & 1 & 0 \\
\hline Kainat & 1 & 0 \\
\hline Kakalij, Rahul M. & 1 & 0 \\
\hline Kakati, Mridusmita & 1 & 0 \\
\hline Kalia, Rajesh & 1 & 0 \\
\hline Kalia, Sapna & 1 & 0 \\
\hline Kalmer, Ramin Ramezani & 1 & 0 \\
\hline Kamal, Hazirah & 1 & 0 \\
\hline Kamal, Mustafa & 1 & 0 \\
\hline Kamel, Medhat M. & 1 & 0 \\
\hline Kamilla, Sushanta Kumar & 1 & 0 \\
\hline Kamyshnyi, Olexandr & 1 & 0 \\
\hline Kanashiro, Milton & 1 & 0 \\
\hline Kanashiro, Milton Masahiko & 1 & 0 \\
\hline Kaptan, Binnur & 1 & 0 \\
\hline Karakurt, Serdar & 1 & 0 \\
\hline Karatzas, Anastasios & 1 & 0 \\
\hline
\end{tabular}




\begin{tabular}{|c|c|c|}
\hline Author & Documents & Citations \\
\hline Karimi, Afzal & 1 & 0 \\
\hline Karpe, Sameer Arvind & 1 & 0 \\
\hline Kartashov, Maksim & 1 & 0 \\
\hline Kasmaee, Mohammad & 1 & 0 \\
\hline Kasmawati, Henny & 1 & 0 \\
\hline Kassaee, Mohammad Zaman & 1 & 0 \\
\hline Kassem, Ahmed & 1 & 0 \\
\hline Kasture, Pooja & 1 & 0 \\
\hline Kasymova, Elvira & 1 & 0 \\
\hline Kazantseva, Tetiana & 1 & 0 \\
\hline Kendrekar, Pravin & 1 & 0 \\
\hline Kerk, Wen Chiann & 1 & 0 \\
\hline Kermani, Ali Sharifzadeh & 1 & 0 \\
\hline Ketharin, Tunasamy & 1 & 0 \\
\hline Khadiv-Parsi, Parisa & 1 & 0 \\
\hline Khafri, Soraya & 1 & 0 \\
\hline Khalid, Azeem & 1 & 0 \\
\hline Khalid, Waqas & 1 & 0 \\
\hline Khalis, Mohammed & 1 & 0 \\
\hline Khan, Ahmad Kamran & 1 & 0 \\
\hline Khan, Ibrahim & 1 & 0 \\
\hline Khanna, Smriti & 1 & 0 \\
\hline Khare, Purnima Swarup & 1 & 0 \\
\hline Khezerloo, Davood & 1 & 0 \\
\hline Khiriya, Pradeep Kumar & 1 & 0 \\
\hline Khodadadi, Effat & 1 & 0 \\
\hline Khorvash, Fariborz & 1 & 0 \\
\hline Khoshgozaran-Abras, Sadegh & 1 & 0 \\
\hline Khoshtinat, Khadijeh & 1 & 0 \\
\hline Khosravi-Darani, Kianosh & 1 & 0 \\
\hline Kiani, Lila & 1 & 0 \\
\hline Kilic, Mehmet & 1 & 0 \\
\hline Kim, Hong Gun & 1 & 0 \\
\hline Kim, Keekyoung & 1 & 0 \\
\hline Kiran, G. & 1 & 0 \\
\hline Klimovitskii, Alexander & 1 & 0 \\
\hline Knut, Ruslan & 1 & 0 \\
\hline Koc, Ziya Erdem & 1 & 0 \\
\hline Kok, Kuan Ying & 1 & 0 \\
\hline Koleva, Milena & 1 & 0 \\
\hline Kolisnyk, Igor & 1 & 0 \\
\hline Kondeva-Burdina, Magdalena & 1 & 0 \\
\hline Kongjan, Prawit & 1 & 0 \\
\hline Konstantin, Uzun & 1 & 0 \\
\hline Kooshki, Hamid & 1 & 0 \\
\hline Koroleva, Lyubov Fedorovna & 1 & 0 \\
\hline Kostova, Irena & 1 & 0 \\
\hline Kotian, Sumana Y. & 1 & 0 \\
\hline Kshanovska, Anna & 1 & 0 \\
\hline Kudrevych, Iryna & 1 & 0 \\
\hline Kudrevych, Oleksandr & 1 & 0 \\
\hline Kudva, Narayana U. N. & 1 & 0 \\
\hline Kuhn, Katia Regina & 1 & 0 \\
\hline Kuhn, Raquel Cristine & 1 & 0 \\
\hline Kukovinets, Olga & 1 & 0 \\
\hline Kulkarni-Almeida, Asha & 1 & 0 \\
\hline Kumar, Akshay & 1 & 0 \\
\hline Kumar, Amarendra & 1 & 0 \\
\hline Kumar, B. Dinesh & 1 & 0 \\
\hline Kumar, J. Rajendra & 1 & 0 \\
\hline Kumar, Tarun & 1 & 0 \\
\hline
\end{tabular}




\begin{tabular}{|c|c|c|}
\hline Author & Documents & Citations \\
\hline Kumari, Karuna & 1 & 0 \\
\hline Kumari, Rosy & 1 & 0 \\
\hline Kunavina, Elena & 1 & 0 \\
\hline Kurajica, Stanislav & 1 & 0 \\
\hline Kuritka, Ivo & 1 & 0 \\
\hline Kuryakova, Tatiana & 1 & 0 \\
\hline Kuryakova, Tatyana & 1 & 0 \\
\hline Kus, Harun & 1 & 0 \\
\hline Kus, Mahmut & 1 & 0 \\
\hline Kushnir, Oksana & 1 & 0 \\
\hline Kwac, Lee $\mathrm{Ku}$ & 1 & 0 \\
\hline Kydralieva, Kamila & 1 & 0 \\
\hline Lasareva, Elena V. & 1 & 0 \\
\hline Lasareva, Elena, V & 1 & 0 \\
\hline Latif, Amirul Faiz Abdul & 1 & 0 \\
\hline Le Cerf, Didier & 1 & 0 \\
\hline Le Thi Minh Chanh & 1 & 0 \\
\hline Leonardo, Ciocca & 1 & 0 \\
\hline Levenets, Tatiana & 1 & 0 \\
\hline Levytska, Svitlana & 1 & 0 \\
\hline Li, Qingqing & 1 & 0 \\
\hline Li, Sergey & 1 & 0 \\
\hline Li, Yunchun & 1 & 0 \\
\hline Liang, Huayin & 1 & 0 \\
\hline Liu, Peili & 1 & 0 \\
\hline Liu, Yuyu & 1 & 0 \\
\hline $\begin{array}{ll}\text { Lokeshwari, } & \text { Devirammanahalli } \\
\text { Mahadevaswamy } & \\
\end{array}$ & 1 & 0 \\
\hline Lolo, Jumiarti Andi & 1 & 0 \\
\hline Lopez-Ramos, Maria & 1 & 0 \\
\hline Lyadov, Nikolay & 1 & 0 \\
\hline Macedo, Ana Angelica M. & 1 & 0 \\
\hline Machunda, Revocatus & 1 & 0 \\
\hline Maciel, Maria A. M. & 1 & 0 \\
\hline Mahadi, Mahani & 1 & 0 \\
\hline Maharramov, Abel Mammadal & 1 & 0 \\
\hline Mahendran, Botlagunta & 1 & 0 \\
\hline Mahmood, Mian Habib-Ur-Rahman & 1 & 0 \\
\hline Mahmood, Qaisar & 1 & 0 \\
\hline Maideen, Naina Mohamed Pakkir & 1 & 0 \\
\hline Makoviichuk, Christina Yaroslavivna & 1 & 0 \\
\hline Makovitzky, Josef & 1 & 0 \\
\hline Maleki, Nariman & 1 & 0 \\
\hline Manciu, Marian & 1 & 0 \\
\hline Mandal, Pinaki & 1 & 0 \\
\hline Mandic, Vilko & 1 & 0 \\
\hline Manzur, Maria E. & 1 & 0 \\
\hline Marcus, Maria-Iuliana & 1 & 0 \\
\hline Mari-Parra, Alexandrea & 1 & 0 \\
\hline Maria, Guillen-Morales & 1 & 0 \\
\hline Marin-Parra, A. & 1 & 0 \\
\hline Marrez, Diaa A. & 1 & 0 \\
\hline Martinez, Karina & 1 & 0 \\
\hline Marzouk, Mohamed Abd El Fattah & 1 & 0 \\
\hline Mashele, Samson & 1 & 0 \\
\hline Masoumi-Ardakani, Yaser & 1 & 0 \\
\hline Matei, Lilia & 1 & 0 \\
\hline Mateu-Armand, Virginia & 1 & 0 \\
\hline Mathiyazhagan, N. & 1 & 0 \\
\hline Maurya, Prem Chandra & 1 & 0 \\
\hline Mazumdar, Himanshu & 1 & 0 \\
\hline
\end{tabular}




\begin{tabular}{|c|c|c|}
\hline Author & Documents & Citations \\
\hline Mazutti, Marcio Antonio & 1 & 0 \\
\hline Mehta, Apurva & 1 & 0 \\
\hline Meliana, Yenny & 1 & 0 \\
\hline Melnychuk, Larysa & 1 & 0 \\
\hline Menegazzo, Federica & 1 & 0 \\
\hline Menevse, Esma & 1 & 0 \\
\hline Meriem, Henni & 1 & 0 \\
\hline Merufinia, Edris & 1 & 0 \\
\hline Mesli, Abderrezzak & 1 & 0 \\
\hline Messaoud, Chaib & 1 & 0 \\
\hline Milani, Abbas & 1 & 0 \\
\hline Mincu, Mariana & 1 & 0 \\
\hline Mindolina, Yulia & 1 & 0 \\
\hline Mirajul-Haq & 1 & 0 \\
\hline Mishra, Bibhuti Bhusan & 1 & 0 \\
\hline Mishra, I. M. & 1 & 0 \\
\hline Mishra, Manswini & 1 & 0 \\
\hline Mishra, Pawan Kumar & 1 & 0 \\
\hline Mishra, Ratish Chandra & 1 & 0 \\
\hline Mishra, Soumya Ranjan & 1 & 0 \\
\hline Mishra, Sujata & 1 & 0 \\
\hline Mobarakeh, M. Iranpour & 1 & 0 \\
\hline Mobini-Dehkordi, Mohsen & 1 & 0 \\
\hline Mochulska, Oksana & 1 & 0 \\
\hline Moghaddam, Arasb Dabbagh & 1 & 0 \\
\hline Moghaddam, Mehrdad Moosazadeh & 1 & 0 \\
\hline Mohamad, Abdul Wahab & 1 & 0 \\
\hline Mohammad, Nurul Aqilah & 1 & 0 \\
\hline Mohammadi, Maedeh & 1 & 0 \\
\hline Mohammadi, Reza & 1 & 0 \\
\hline Mohammadi-Samani, Soliman & 1 & 0 \\
\hline Mohammadian, Mehdi & 1 & 0 \\
\hline Mohapatra, Priyaranjan & 1 & 0 \\
\hline Montalvo, Gemma & 1 & 0 \\
\hline Moraveji, Mostafa Keshavarz & 1 & 0 \\
\hline Mores, Rubia & 1 & 0 \\
\hline Morillo, Marielba & 1 & 0 \\
\hline Moroz, Petro, V & 1 & 0 \\
\hline Mortazavi, Ali & 1 & 0 \\
\hline Mostafa, Amr Ahmed & 1 & 0 \\
\hline Mostafa, Dina & 1 & 0 \\
\hline Moulai-Mostefa, Nadji & 1 & 0 \\
\hline Mudarisova, Roza & 1 & 0 \\
\hline Muhamad, Mimi Suliza & 1 & 0 \\
\hline Mukhametzyanov, Timur & 1 & 0 \\
\hline Mulyani, Evi & 1 & 0 \\
\hline Murthy, Srinivasa, V & 1 & 0 \\
\hline Musetescu, Alina & 1 & 0 \\
\hline Mustapha, Siti Noor Hidayah & 1 & 0 \\
\hline Muthusamy, R. & 1 & 0 \\
\hline Mykhailovych, Andreychyn Serhiy & 1 & 0 \\
\hline Nadh, R. Venkata & 1 & 0 \\
\hline Naeimi, Mitra & 1 & 0 \\
\hline Najafpour, Ghasem & 1 & 0 \\
\hline Namazov, Nizami R. & 1 & 0 \\
\hline Naseri, Farnoosh & 1 & 0 \\
\hline Nasiri, Alireza & 1 & 0 \\
\hline Nassari, Hani & 1 & 0 \\
\hline Nayak, Rasmita & 1 & 0 \\
\hline Nazarova, Marina Yu. & 1 & 0 \\
\hline Nazarparvar, Melika & 1 & 0 \\
\hline
\end{tabular}




\begin{tabular}{|c|c|c|}
\hline Author & Documents & Citations \\
\hline Nedelcu, Daniela & 1 & 0 \\
\hline Negahdari, Ramin & 1 & 0 \\
\hline Nejati-Shendi, Mohammad & 1 & 0 \\
\hline Nikolaevna, Ivanova Svetlana & 1 & 0 \\
\hline Nikyfor, Livia & 1 & 0 \\
\hline Noh-Delgado, Raquel & 1 & 0 \\
\hline Nomura, Kiyoshi & 1 & 0 \\
\hline Noori, Allahyar & 1 & 0 \\
\hline Noormand, Fatemeh & 1 & 0 \\
\hline Novikov, Volodymyr & 1 & 0 \\
\hline Novoselov, Igor & 1 & 0 \\
\hline Nunez-Vallejos, Diego-Alejandro & 1 & 0 \\
\hline Nurmawati, Oktovia & 1 & 0 \\
\hline Nursamsiar & 1 & 0 \\
\hline Okudan, Nilsel & 1 & 0 \\
\hline Olehovych, Dobrianskyi Taras & 1 & 0 \\
\hline Olevska, Yuliia & 1 & 0 \\
\hline Olevskyi, Viktor & 1 & 0 \\
\hline Olga, Kvan, V & 1 & 0 \\
\hline Omar, Fuaad & 1 & 0 \\
\hline Ortiz, Camilo & 1 & 0 \\
\hline Ozalp, Coskun & 1 & 0 \\
\hline Packiaraj, G. & 1 & 0 \\
\hline Pal, Anita & 1 & 0 \\
\hline Palabiyik, Ibrahim & 1 & 0 \\
\hline Panda, Snehashis & 1 & 0 \\
\hline Panus, Emilia & 1 & 0 \\
\hline Paolo, Baldissara & 1 & 0 \\
\hline Paolo, Mora & 1 & 0 \\
\hline Parikesit, Arli Aditya & 1 & 0 \\
\hline Parizi, Moazameh Alidad & 1 & 0 \\
\hline Parnia, Feridoun & 1 & 0 \\
\hline Parul & 1 & 0 \\
\hline Pasechnik, Tatiana & 1 & 0 \\
\hline Pashkovska, Natalia Viktoravna & 1 & 0 \\
\hline Patamia, Ildebrando & 1 & 0 \\
\hline Patra, Swarna Mayee & 1 & 0 \\
\hline Pattanaik, Priyabrata & 1 & 0 \\
\hline Paul, Parimal & 1 & 0 \\
\hline Paulraj, Ponnaiah & 1 & 0 \\
\hline Pawar, Rajandra & 1 & 0 \\
\hline Perara, Sudarshana Jayaraj & 1 & 0 \\
\hline Petkova, Nadezhda & 1 & 0 \\
\hline Petrynych, Oksana & 1 & 0 \\
\hline Pezzino, Salvatore & 1 & 0 \\
\hline Phule, Ajit Dattatreya & 1 & 0 \\
\hline Piatnochka, Volodymyr, I & 1 & 0 \\
\hline Piddubna, Antonina Natolivna & 1 & 0 \\
\hline Pitsikalis, Marinos & 1 & 0 \\
\hline Pizzolitto, Cristina & 1 & 0 \\
\hline Plume, Imants & 1 & 0 \\
\hline Pogrebnaya, Tatiana & 1 & 0 \\
\hline Polianskiy, Ihor Iu & 1 & 0 \\
\hline Polina, Lapsina V. & 1 & 0 \\
\hline Ponomareva, Polina & 1 & 0 \\
\hline Pool Gomez, Eduardo & 1 & 0 \\
\hline Popletaeva, Sofya & 1 & 0 \\
\hline Popov, Valerii & 1 & 0 \\
\hline Pourbagher-Shahri, Ali Mohammad & 1 & 0 \\
\hline Pournasrollah, Alireza & 1 & 0 \\
\hline Pradhan, Mamata & 1 & 0 \\
\hline
\end{tabular}




\begin{tabular}{|c|c|c|}
\hline Author & Documents & Citations \\
\hline Pradhan, Manas Ranjan & 1 & 0 \\
\hline Pradhan, Sanghamitra & 1 & 0 \\
\hline Pramanik, Goutam & 1 & 0 \\
\hline Pramanik, Susmita & 1 & 0 \\
\hline Pramanik, Tanay & 1 & 0 \\
\hline Prokhorenko, Victor & 1 & 0 \\
\hline Pu'ad, Nor Atiqah Syafinaz Mohd & 1 & 0 \\
\hline Puglisi, Fabrizio & 1 & 0 \\
\hline Puspitasari, Ratih Dyah & 1 & 0 \\
\hline Putri, Rahmawati & 1 & 0 \\
\hline Pytka, Alex S. & 1 & 0 \\
\hline Qi Zhang & 1 & 0 \\
\hline Raad, Haidar Taimouri & 1 & 0 \\
\hline Rabbani, Hossein & 1 & 0 \\
\hline Radwan, Mostafa A. & 1 & 0 \\
\hline Radwan, Sara H. & 1 & 0 \\
\hline Rahbar, Mahdi & 1 & 0 \\
\hline Rahman, Atiq Ur & 1 & 0 \\
\hline Rahman, Md. Mominur & 1 & 0 \\
\hline Rai, K. M. Lokanatha & 1 & 0 \\
\hline Raja, Iftikhar & 1 & 0 \\
\hline Rajendrachari, Shashanka & 1 & 0 \\
\hline Rakhmatullin, Shamil & 1 & 0 \\
\hline Ramazanov, Mahammadali A. & 1 & 0 \\
\hline Ramazanov, Mahammadali Ahmad & 1 & 0 \\
\hline Ramli, Siti Zulaikha & 1 & 0 \\
\hline Ranjbariyan, Alireza & 1 & 0 \\
\hline Rao, Nagasarapu Mallikharjuna & 1 & 0 \\
\hline Rashwan, Salah M. & 1 & 0 \\
\hline Rasooli, Iraj & 1 & 0 \\
\hline Ravinder, D. & 1 & 0 \\
\hline Raviz, Elahe Karami & 1 & 0 \\
\hline Ray, Pratima & 1 & 0 \\
\hline Reddy, Golamari Siva & 1 & 0 \\
\hline Reddy, Ronda Srinivasa & 1 & 0 \\
\hline Reihani, Seyedeh Fatemeh Seyed & 1 & 0 \\
\hline Rejeb, Randa & 1 & 0 \\
\hline Repchuk, Yulia & 1 & 0 \\
\hline Reverchon, Ernesto & 1 & 0 \\
\hline Rezaei, Rasoul & 1 & 0 \\
\hline Rezaeipour, Ebrahim & 1 & 0 \\
\hline Rezgui, Nacer & 1 & 0 \\
\hline Rezvani, Majid & 1 & 0 \\
\hline Rimpy & 1 & 0 \\
\hline Rivera, David & 1 & 0 \\
\hline Rizvi, Zejah & 1 & 0 \\
\hline Robaina-Mesa, Malvis & 1 & 0 \\
\hline Rodenas, Elvira & 1 & 0 \\
\hline Rodriguez Chanfrau, Jorge E. & 1 & 0 \\
\hline Rodriguez Chanfrau, Jorge Enrique & 1 & 0 \\
\hline Rojas-Fermin, Luis & 1 & 0 \\
\hline Romli, Ahmad Zafir & 1 & 0 \\
\hline Rosas-Nexticapa, Marcela & 1 & 0 \\
\hline Roshani, Kimia & 1 & 0 \\
\hline Rosoiu, Natalia & 1 & 0 \\
\hline Rouhi, Yashar & 1 & 0 \\
\hline Rout, Ardhendu Sekhar & 1 & 0 \\
\hline Roy, Apurba Kumar & 1 & 0 \\
\hline Rudyk, Roxana A. & 1 & 0 \\
\hline Rusciano, Dario & 1 & 0 \\
\hline Ruslin & 1 & 0 \\
\hline
\end{tabular}




\begin{tabular}{|c|c|c|}
\hline Author & Documents & Citations \\
\hline Rykov, Alexandre I. & 1 & 0 \\
\hline Rzaeva, Afak L. & 1 & 0 \\
\hline Rzayev, Fuad H. & 1 & 0 \\
\hline Sabri, Nadjia & 1 & 0 \\
\hline Sabrina, Gioria & 1 & 0 \\
\hline Sachin, K. M. & 1 & 0 \\
\hline Sadak, Abderrahim & 1 & 0 \\
\hline Saeed, Bahjat & 1 & 0 \\
\hline Saffar-Teluri, A. & 1 & 0 \\
\hline Sagitova, Alina & 1 & 0 \\
\hline Saha, Petr & 1 & 0 \\
\hline Sahoo, Priyadarshini Shivani & 1 & 0 \\
\hline Sahoo, Subrat Kumar & 1 & 0 \\
\hline Sahumena, Muh Handoyo & 1 & 0 \\
\hline Sailaja, Muchipali & 1 & 0 \\
\hline Sajeesh, Pattammadath & 1 & 0 \\
\hline Salah, Salwa & 1 & 0 \\
\hline Salamanca, Constain H. & 1 & 0 \\
\hline Salman, Nor Syazwani & 1 & 0 \\
\hline Samini, Fariborz & 1 & 0 \\
\hline Sane, Zhu & 1 & 0 \\
\hline Sangtarash, Mohammad Hossein & 1 & 0 \\
\hline Sanjeev, Airy & 1 & 0 \\
\hline Sankar, Dannana Gowri & 1 & 0 \\
\hline Sapka, Marianthi & 1 & 0 \\
\hline Sara, Ragazzini & 1 & 0 \\
\hline Sarangi, Dayananda & 1 & 0 \\
\hline Sarkar, Prosanta & 1 & 0 \\
\hline Sarmah, Bidyut Kumar & 1 & 0 \\
\hline Sehil, Hafida & 1 & 0 \\
\hline Seifi, Mohammad & 1 & 0 \\
\hline Seijo-Santos, Angel & 1 & 0 \\
\hline Selimin, Mohamad Ali & 1 & 0 \\
\hline Semenovich, Markvichev Nikolay & 1 & 0 \\
\hline Semianiv, Marianna & 1 & 0 \\
\hline Septiyanti, Melati & 1 & 0 \\
\hline Shaghayegh, Gohar & 1 & 0 \\
\hline Shah, Dawood & 1 & 0 \\
\hline Shah, Fayyaz Ali & 1 & 0 \\
\hline Shah, Sayed Suliman & 1 & 0 \\
\hline Shahin, Asmaa & 1 & 0 \\
\hline Shahpar, Amir Hossein & 1 & 0 \\
\hline Shahrabi, Mohammadjavad Ameri & 1 & 0 \\
\hline Shakarami, Zahra & 1 & 0 \\
\hline Shako, Alireza & 1 & 0 \\
\hline Shalaby, Eman & 1 & 0 \\
\hline Shalaby, Rizk Moustafa & 1 & 0 \\
\hline Shams, Akbar & 1 & 0 \\
\hline Shanmugam, Venkat Kumar & 1 & 0 \\
\hline Shanthakumar, Dileep C. & 1 & 0 \\
\hline Shao, Xiaolin & 1 & 0 \\
\hline Sharifan, Anousheh & 1 & 0 \\
\hline Sharifi, Esmaeel & 1 & 0 \\
\hline Sharma, J. K. & 1 & 0 \\
\hline Sharma, Manisha & 1 & 0 \\
\hline Sharma, Promod Kumar & 1 & 0 \\
\hline Sharma, Rajiv & 1 & 0 \\
\hline Sharma, Sangita & 1 & 0 \\
\hline Sharma, Shweta & 1 & 0 \\
\hline Shaygannejad, Vahid & 1 & 0 \\
\hline Shcherbakova, Larisa & 1 & 0 \\
\hline
\end{tabular}




\begin{tabular}{|c|c|c|}
\hline Author & Documents & Citations \\
\hline Shendabadi, Alireza Rezazadeh & 1 & 0 \\
\hline Shende, Pravin & 1 & 0 \\
\hline Sheoran, Ruchika & 1 & 0 \\
\hline Sheremet, Michael & 1 & 0 \\
\hline Sheremet, Michael Ivanovich & 1 & 0 \\
\hline Shridevi, D. & 1 & 0 \\
\hline Shuba, Dmytro & 1 & 0 \\
\hline Shukla, Vinita & 1 & 0 \\
\hline Sidashenko, Olga & 1 & 0 \\
\hline Siddique, Md Nurul Islam & 1 & 0 \\
\hline Sidorenko, Galina & 1 & 0 \\
\hline Signoretto, Michela & 1 & 0 \\
\hline Silva, Cleber C. & 1 & 0 \\
\hline Silva, Maria S. & 1 & 0 \\
\hline Silva, Thayna Souza & 1 & 0 \\
\hline Simonneaux, Gerard & 1 & 0 \\
\hline Sinesi, Antonia & 1 & 0 \\
\hline Singh, Kamaldeep & 1 & 0 \\
\hline Singh, Man & 1 & 0 \\
\hline Singh, Shefali & 1 & 0 \\
\hline Sizentsov, Alexey & 1 & 0 \\
\hline Sokolenko, Alina & 1 & 0 \\
\hline Sokolenko, Ludmila & 1 & 0 \\
\hline Sombra, Antonio S. B. & 1 & 0 \\
\hline Soreshjani, Sedigheh Abdollahzadeh & 1 & 0 \\
\hline Sotnikov, Dmitriy, V & 1 & 0 \\
\hline Soyler, Mustafa & 1 & 0 \\
\hline Srinivasulu, Kamma & 1 & 0 \\
\hline Srivastava, V. C. & 1 & 0 \\
\hline Statsyuk, Natalia & 1 & 0 \\
\hline Straume, Indulis & 1 & 0 \\
\hline Su, Lan & 1 & 0 \\
\hline Sukhodolska, Natalia & 1 & 0 \\
\hline Sulyma, Marta & 1 & 0 \\
\hline Sun, Xueliang & 1 & 0 \\
\hline Suratno & 1 & 0 \\
\hline Suresh, K. & 1 & 0 \\
\hline Suresh, S. & 1 & 0 \\
\hline Svahn, Isabelle & 1 & 0 \\
\hline Svetlana, Zamana P. & 1 & 0 \\
\hline Swain, Nandita & 1 & 0 \\
\hline Swapna, B. & 1 & 0 \\
\hline Sydorchuk, Andrii & 1 & 0 \\
\hline Sydorchuk, Larysa & 1 & 0 \\
\hline Sydorchuk, Ruslan & 1 & 0 \\
\hline Syukur, Elsa Ofta Sari & 1 & 0 \\
\hline Tabarkiewicz, Jacek & 1 & 0 \\
\hline Tabrizi, Sina Alizadeh & 1 & 0 \\
\hline Tahani, Soheil & 1 & 0 \\
\hline Tahmasebi, Elahe & 1 & 0 \\
\hline Talbaoui, Ahmed & 1 & 0 \\
\hline Talmaci, Rodica & 1 & 0 \\
\hline Talpur, Mir Zulqarnain & 1 & 0 \\
\hline Tamoshina, Olga & 1 & 0 \\
\hline Tan, Huey Ling & 1 & 0 \\
\hline Teiwes, Hanno & 1 & 0 \\
\hline Tekale, Sunil & 1 & 0 \\
\hline Teodorescu, Irina & 1 & 0 \\
\hline Tezerji, Najmeh Sheibani & 1 & 0 \\
\hline Thakur, Ajay Kumar & 1 & 0 \\
\hline Thiruvengadam, Veeraswamy & 1 & 0 \\
\hline
\end{tabular}




\begin{tabular}{|c|c|c|}
\hline Author & Documents & Citations \\
\hline Tian, Changan & 1 & 0 \\
\hline Tibullo, Daniele & 1 & 0 \\
\hline Timchy, Kateryna & 1 & 0 \\
\hline Tiomno-Tiomnova, Olinka & 1 & 0 \\
\hline Titkova, Anna & 1 & 0 \\
\hline Todero, Izelmar & 1 & 0 \\
\hline Todorov, Lozan & 1 & 0 \\
\hline Toma, Diana & 1 & 0 \\
\hline Tomas Joel, Lopez-Gutierrez & 1 & 0 \\
\hline Torab, Ali & 1 & 0 \\
\hline Tosun, Gul & 1 & 0 \\
\hline Tres, Marcus & 1 & 0 \\
\hline Tres, Marcus Vinicius & 1 & 0 \\
\hline Tres, Marcus, V & 1 & 0 \\
\hline Tripathi, Smriti Ojha & 1 & 0 \\
\hline Tripathy, Abhisheck Dutt & 1 & 0 \\
\hline Tripathy, Arpita & 1 & 0 \\
\hline Tripathy, Hara Prasada & 1 & 0 \\
\hline Trivedi, Mahendra Kumar & 1 & 0 \\
\hline Turkoglu, Emir Alper & 1 & 0 \\
\hline Tutka, Piotr & 1 & 0 \\
\hline Tzankova, Virginia & 1 & 0 \\
\hline Usman, Muhammad & 1 & 0 \\
\hline Usubillaga, Alfredo & 1 & 0 \\
\hline Vadim, Dodonov G. & 1 & 0 \\
\hline Vafaiee, Mohaddeseh & 1 & 0 \\
\hline Valentynovych, Zhulkevych Igor & 1 & 0 \\
\hline Valerino-Diaz, Alexander & 1 & 0 \\
\hline Valiente, Mercedes & 1 & 0 \\
\hline Varaminian, Farshad & 1 & 0 \\
\hline Vasilache, Didona & 1 & 0 \\
\hline Vassilev, Dragomir & 1 & 0 \\
\hline Vasudha, B. & 1 & 0 \\
\hline Veliyeva, Solmaz B. & 1 & 0 \\
\hline Venkatesan, Arthi & 1 & 0 \\
\hline Venquiaruto, Luciana Dornelles & 1 & 0 \\
\hline Verma, Shakuntla & 1 & 0 \\
\hline Vicas, C. S. & 1 & 0 \\
\hline Vilhena, Felipe S. & 1 & 0 \\
\hline Vilhena, Fernando S. D. S. & 1 & 0 \\
\hline Vladimir, Kolpakov & 1 & 0 \\
\hline Vladimirovich, Kalenov Sergei & 1 & 0 \\
\hline Voinova, Tatiana & 1 & 0 \\
\hline Voroniuk, Ksenia & 1 & 0 \\
\hline Vrancianu, Corneliu Ovidiu & 1 & 0 \\
\hline Wagutu, Agatha W. & 1 & 0 \\
\hline Walpalage, Shantha & 1 & 0 \\
\hline Wang, Junhu & 1 & 0 \\
\hline Wang, Xiangming & 1 & 0 \\
\hline Wee, Boon Siong & 1 & 0 \\
\hline Wirasutisna, Komar Ruslan & 1 & 0 \\
\hline Xu, Liping & 1 & 0 \\
\hline Yadav, Jaya Parkash & 1 & 0 \\
\hline Yadav, Krishna Kant & 1 & 0 \\
\hline Yan, Zhao & 1 & 0 \\
\hline Yao, Jie & 1 & 0 \\
\hline Yaroslav, Sizentsov A. & 1 & 0 \\
\hline Yaroslavivna, Fedoniuk Larysa & 1 & 0 \\
\hline Yarynych, Yulia & 1 & 0 \\
\hline Yasar, Kurban & 1 & 0 \\
\hline Yasmeen, Nafeesa & 1 & 0 \\
\hline
\end{tabular}




\begin{tabular}{l|l|l} 
Author & Documents & Citations \\
\hline Yausheva, Elena & 1 & 0 \\
\hline Yazdani, Javad & 1 & 0 \\
\hline Yilmaztekin, Murat & 1 & 0 \\
\hline Yin, Qiyi & 1 & 0 \\
\hline Yousaf, Muhammad & 1 & 0 \\
\hline Yousefi, Mojtaba & 1 & 0 \\
\hline Yusifova, Anahanum A. & 1 & 0 \\
\hline Zabot, Giovani L. & 1 & 0 \\
\hline Zaher, Amira & 1 & 0 \\
\hline Zahir, Muhammad & 1 & 0 \\
\hline Zamani, Saeed & 1 & 0 \\
\hline Zamri, Siti Sarah & 1 & 0 \\
\hline Zeini, Mohsen & 1 & 0 \\
\hline Zengin, Gokhan & 1 & 0 \\
\hline Zernov, Anton & 1 & 0 \\
\hline Zhang Xurui & 1 & 0 \\
\hline Zhang, Jiujun & 1 & 0 \\
\hline Zhang, Lizhi & 1 & 0 \\
\hline Zhang, Xiangjun & 1 & 0 \\
\hline Zhebo, Anna & 1 & 0 \\
\hline Zia, Huma & 1 & 0 \\
\hline Ziganshin, Marat & 1 & 0 \\
\hline Zihayat, Bahare & 1 & 0 \\
\hline Zolfaghari, Davood & 1 & 0 \\
\hline Zulkifli, Zulfah & 1 & 0 \\
\hline Zurac, Sabina & 1 & 0 \\
\hline & &
\end{tabular}

Table S2. List of 838 institutes with total documents and citations.

Organization

\begin{tabular}{|c|c|c|}
\hline Organızatıon & Documents & Citations \\
\hline Islamic Azad Univ & 77 & 226 \\
\hline Natl Res Ctr & 46 & 155 \\
\hline Ain Shams Univ & 23 & 114 \\
\hline Univ Tehran Med Sci & 13 & 81 \\
\hline Aligarh Muslim Univ & 5 & 61 \\
\hline Univ Bucharest & 26 & 46 \\
\hline Tabriz Univ Med Sci & 16 & 43 \\
\hline Amirkabir Univ Technol & 13 & 41 \\
\hline Gitam Deemed Be Univ & 9 & 39 \\
\hline Russian Acad Sci & 18 & 30 \\
\hline Shahid Beheshti Univ Med Sci & 16 & 29 \\
\hline Beni Suef Univ & 2 & 29 \\
\hline Cairo Univ & 10 & 27 \\
\hline British Univ Egypt & 5 & 27 \\
\hline Mansoura Univ & 9 & 23 \\
\hline Univ Tehran & 8 & 23 \\
\hline Shahid Sadoughi Univ Med Sci & 4 & 23 \\
\hline Curtin Univ & 7 & 22 \\
\hline Mat \& Energy Res Ctr Merc & 4 & 22 \\
\hline Univ Teknol Malaysia & 12 & 21 \\
\hline King Saud Univ & 8 & 21 \\
\hline Osmania Univ & 4 & 21 \\
\hline King Khalid Univ & 6 & 20 \\
\hline Univ Bucharest Icub & 3 & 19 \\
\hline Danylo Halytsky Lviv Natl Med Univ & 5 & 18 \\
\hline Ivan Franko Natl Univ Lviv & 5 & 18 \\
\hline Univ Tabriz & 7 & 16 \\
\hline Birla Inst Technol & 3 & 16 \\
\hline Iran Univ Med Sci & 3 & 16 \\
\hline Cent Reference Synevo Lab Romania & 2 & 16 \\
\hline Shiraz Univ Med Sci & 9 & 15 \\
\hline Univ Nacl Tucuman & 6 & 15 \\
\hline Jntua & 2 & 15 \\
\hline Chung Yuan Christian Univ & 1 & 15 \\
\hline Jamia Millia Islamia & 1 & 15 \\
\hline
\end{tabular}




\section{Organization}

Natl Inst Cardiovasc Dis Prof Cc Iliescu

$\mathrm{Nrc}$

Inst Teknol Sepuluh Nopember

Univ Vale Rio Doce

Minist Hlth Russia

Sri Krishnadevaraya Univ

Univ Mysore

Sharif Univ Technol

Amer Univ Cairo

Bulgarian Acad Sci

Majmaah Univ Al Majmaah

Vignans Inst Engn Women

Ege Univ

Mashhad Univ Med Sci

Natl Inst Oceanog \& Fisheries

Shaheed Benazir Bhutto Women Univ

Univ Oil \& Gas

Univ Peshawar

Univ Sains Malaysia

Ural State Univ Econ

Ggdsd Coll

Univ Dunrea De Jos

Baqiyatallah Univ Med Sci

Bandung Inst Technol

Birjand Univ Med Sci

Carol Davila Univ Med \& Pharm

Emergency Univ Hosp

Hacettepe Univ

Neyshabur Univ Med Sci

Vellore Inst Technol

Univ Reg Integrada Alto Uruguai \& Missoes

Vietnam Natl Univ Ho Chi Minh City

Glasgow Caledonian Univ

Bhupal Nobles Post Grad Bnpg Coll

Inst Sci \& Technol Polymers

Shahidsadoughi Univ Med Sci

Sofia Univ St Kliment Ohridski

Univ Chem Technol \& Metallurgy

Selcuk Univ

Univ Texas El Paso

Lhokseumawe State Polytech

Mayo Clin

Vietnam Natl Univ

Thiru Kollanjiappar Arts Coll

Natl Tsing Hua Univ

Nriag

Quaid I Azam Univ

Vietnam Natl Univ Ho Chi Minh City Vnuhcm

Djilali Liabes Univ

Uttaranchal Univ

Abant Izzet Baysal Univ

Arena Arbeitsgemeinschaft Ressourcenschonende \& N

Fed Univ Sao Carlos Ufscar

Graz Univ

Imec

Nawi Graz

Shahid Beheshti Univ

Torbat Heydariyeh Univ Med Sci

Univ Ghent

Univ Sistan \& Baluchestan

Velayat Univ

Gla Univ

Vit Univ

Alexandria Univ

Maragheh Univ Med Sci

Northeastern Univ

\begin{tabular}{|c|c|}
\hline Documents & Citations \\
\hline 3 & 14 \\
\hline 3 & 14 \\
\hline 8 & 13 \\
\hline 2 & 13 \\
\hline 1 & 13 \\
\hline 1 & 13 \\
\hline 8 & 12 \\
\hline 4 & 12 \\
\hline 3 & 12 \\
\hline 2 & 12 \\
\hline 1 & 12 \\
\hline 1 & 12 \\
\hline 3 & 11 \\
\hline 3 & 11 \\
\hline 3 & 11 \\
\hline 2 & 11 \\
\hline 2 & 11 \\
\hline 2 & 11 \\
\hline 2 & 11 \\
\hline 2 & 11 \\
\hline 1 & 11 \\
\hline 1 & 11 \\
\hline 7 & 10 \\
\hline 5 & 10 \\
\hline 5 & 10 \\
\hline 5 & 10 \\
\hline 5 & 10 \\
\hline 5 & 10 \\
\hline 5 & 10 \\
\hline 5 & 10 \\
\hline 3 & 10 \\
\hline 3 & 10 \\
\hline 2 & 10 \\
\hline 1 & 10 \\
\hline 1 & 10 \\
\hline 1 & 10 \\
\hline 1 & 10 \\
\hline 1 & 10 \\
\hline 9 & 9 \\
\hline 4 & 9 \\
\hline 3 & 9 \\
\hline 3 & 9 \\
\hline 3 & 9 \\
\hline 2 & 9 \\
\hline 1 & 9 \\
\hline 1 & 9 \\
\hline 6 & 8 \\
\hline 4 & 8 \\
\hline 3 & 8 \\
\hline 3 & 8 \\
\hline 2 & 8 \\
\hline 1 & 8 \\
\hline 1 & 8 \\
\hline 1 & 8 \\
\hline 1 & 8 \\
\hline 1 & 8 \\
\hline 1 & 8 \\
\hline 1 & 8 \\
\hline 1 & 8 \\
\hline 6 & 7 \\
\hline 5 & 7 \\
\hline 4 & 7 \\
\hline 4 & 7 \\
\hline 3 & 7 \\
\hline 3 & 7 \\
\hline 3 & 7 \\
\hline
\end{tabular}




\begin{tabular}{|c|c|c|}
\hline Organization & Documents & Citations \\
\hline Univ Hail & 3 & 7 \\
\hline Landmark Univ & 2 & 7 \\
\hline Univ Fed Mato Grosso & 2 & 7 \\
\hline Univ Fed Sao Carlos & 2 & 7 \\
\hline Vtpchem Pty Ltd & 2 & 7 \\
\hline Ada Fdn & 1 & 7 \\
\hline Amu & 1 & 7 \\
\hline Dubna State Univ & 1 & 7 \\
\hline Fed Univ & 1 & 7 \\
\hline Fed Univ Matogrosso & 1 & 7 \\
\hline Fed Univ Matogrosso Do Sul & 1 & 7 \\
\hline $\mathrm{Icm}$ & 1 & 7 \\
\hline Masaryk Univ Brno & 1 & 7 \\
\hline Menoufia Univ & 1 & 7 \\
\hline Natl Res Ctr Hbrc & 1 & 7 \\
\hline Univ Andres Bello & 1 & 7 \\
\hline Univ Catolica Norte & 1 & 7 \\
\hline Univ Fed Abc & 1 & 7 \\
\hline Univ Fed Sao Paulo & 1 & 7 \\
\hline Witec Gmbh & 1 & 7 \\
\hline Al Azhar Univ & 5 & 6 \\
\hline Univ Estadual Paulista & 5 & 6 \\
\hline Yunnan Univ & 4 & 6 \\
\hline Daffodil Int Univ & 3 & 6 \\
\hline Ferdowsi Univ Mashhad & 3 & 6 \\
\hline Shahed Univ & 3 & 6 \\
\hline Univ Fed Mato Grosso Do Sul & 3 & 6 \\
\hline Univ Malaysia Pahang & 3 & 6 \\
\hline Aalborg Univ & 2 & 6 \\
\hline Anna Univ Chennai & 2 & 6 \\
\hline Arignar Anna Govt Arts Coll & 2 & 6 \\
\hline Bharathiar Univ & 2 & 6 \\
\hline Cent Glass \& Ceram Res Inst & 2 & 6 \\
\hline Mawlana Bhashani Sci \& Technol Univ Mbstu & 2 & 6 \\
\hline Sree Krishna Coll Engn & 2 & 6 \\
\hline Thiruvalluvar Univ & 2 & 6 \\
\hline All Saints Univ & 1 & 6 \\
\hline Islamic Azad Med Univ & 1 & 6 \\
\hline Marmara Univ & 1 & 6 \\
\hline Mcpherson Univ & 1 & 6 \\
\hline Tabriz Univ & 1 & 6 \\
\hline Univ Palermo & 1 & 6 \\
\hline Univ Pendidikan Indonesia & 1 & 6 \\
\hline Vit & 1 & 6 \\
\hline Siksha O Anusandhan Deemed Be Univ & 10 & 5 \\
\hline Sathyabama Inst Sci \& Technol & 4 & 5 \\
\hline Comsats Univ Islamabad & 3 & 5 \\
\hline King Abdulaziz Univ & 3 & 5 \\
\hline Al Balqa Univ & 2 & 5 \\
\hline Its Coll Pharm & 2 & 5 \\
\hline Jouf Univ & 2 & 5 \\
\hline Jss Coll Arts Commerce \& Sci & 2 & 5 \\
\hline Res \& Dev Inst Environm Protect & 2 & 5 \\
\hline Aarhus Univ & 1 & 5 \\
\hline Birla Inst Technol Mesra & 1 & 5 \\
\hline Concordia Univ & 1 & 5 \\
\hline Fiocruz Minas & 1 & 5 \\
\hline Haeri Univ Meybod & 1 & 5 \\
\hline Indian Inst Chem Biol & 1 & 5 \\
\hline Integrated Pharmacol \& Drug Interact Res Grp Gpqf & 1 & 5 \\
\hline Natl Inst Technol Silchar & 1 & 5 \\
\hline Sadat Univ & 1 & 5 \\
\hline Santo Agostinho Inst & 1 & 5 \\
\hline Sci \& Art Univ & 1 & 5 \\
\hline Univ Dunarea De Jos & 1 & 5 \\
\hline Univ Khartoum & 1 & 5 \\
\hline
\end{tabular}




\begin{tabular}{|c|c|c|}
\hline Organization & Documents & Citations \\
\hline Univ Taif & 1 & 5 \\
\hline Anurag Grp Inst & 4 & 4 \\
\hline Inonu Univ & 4 & 4 \\
\hline Osmaniye Korkut Ata Univ & 4 & 4 \\
\hline Tezpur Univ & 4 & 4 \\
\hline Univ Catania & 4 & 4 \\
\hline Univ Malaysia Terengganu & 4 & 4 \\
\hline Helwan Univ & 3 & 4 \\
\hline Shahid Bahonar Univ Kerman & 3 & 4 \\
\hline Univ Indonesia & 3 & 4 \\
\hline Al Nahrain Univ & 2 & 4 \\
\hline Gitam Deemed Univ & 2 & 4 \\
\hline Natl Inst Genet Engn \& Biotechnol & 2 & 4 \\
\hline Prince Songkla Univ & 2 & 4 \\
\hline Sao Paulo State Univ & 2 & 4 \\
\hline Sri Jayachamarajendra Coll Engn & 2 & 4 \\
\hline Univ Nizwa & 2 & 4 \\
\hline Abetaia & 1 & 4 \\
\hline Actr Univ Ain Temouchent Dgrct & 1 & 4 \\
\hline Bangor Univ & 1 & 4 \\
\hline Bhabha Atom Res Ctr & 1 & 4 \\
\hline Cirad & 1 & 4 \\
\hline Comsats Univ Islamabad Cui & 1 & 4 \\
\hline $\mathrm{Cttm}$ & 1 & 4 \\
\hline Curtin Univ Malaysia & 1 & 4 \\
\hline Dicle Univ & 1 & 4 \\
\hline Dongguk Univ & 1 & 4 \\
\hline Hazara Univ Mansehra & 1 & 4 \\
\hline Inst Nanotechnol Int & 1 & 4 \\
\hline Irccs San Raffaele Pisana & 1 & 4 \\
\hline Maharanis Sci Coll Women & 1 & 4 \\
\hline Megarezky Univ & 1 & 4 \\
\hline Near East Univ & 1 & 4 \\
\hline Pineta Grande Private Hosp & 1 & 4 \\
\hline Sies Coll Arts Sci \& Commerce & 1 & 4 \\
\hline Univ Americas Puebla & 1 & 4 \\
\hline Univ Azad Jammu \& Kashmir & 1 & 4 \\
\hline Univ Brunei Darussalam & 1 & 4 \\
\hline Univ Islam Madura & 1 & 4 \\
\hline Univ Pittsburgh & 1 & 4 \\
\hline Univ Salerno Italy & 1 & 4 \\
\hline Ural Fed Univ & 1 & 4 \\
\hline Vasile Goldis Western Univ Arad & 1 & 4 \\
\hline Vignan Pharm Coll & 1 & 4 \\
\hline Villa Fiorita Private Hosp & 1 & 4 \\
\hline Univ Autonomous Campeche & 17 & 3 \\
\hline Univ Veracruzana & 17 & 3 \\
\hline Kerman Univ Med Sci & 6 & 3 \\
\hline Mansinhbhai Inst Dairy \& Food Technol & 5 & 3 \\
\hline Vidyavardhaka Coll Engn & 4 & 3 \\
\hline Firat Univ & 3 & 3 \\
\hline Univ Agr & 3 & 3 \\
\hline Univ Djillali Liabes Sidi Bel Abbes & 3 & 3 \\
\hline Abdul Wali Khan Univ Mardan & 2 & 3 \\
\hline Akdeniz Univ & 2 & 3 \\
\hline Chulalongkorn Univ & 2 & 3 \\
\hline Comsats Inst Informat Technol & 2 & 3 \\
\hline Govt Coll Univ & 2 & 3 \\
\hline Graph Era Deemed Be Univ & 2 & 3 \\
\hline Kermanshah Univ Med Sci & 2 & 3 \\
\hline Mutah Univ & 2 & 3 \\
\hline Necmettin Erbakan Univ & 2 & 3 \\
\hline Shiraz Univ & 2 & 3 \\
\hline Suranaree Univ Technol & 2 & 3 \\
\hline Tanta Univ & 2 & 3 \\
\hline Univ Havana & 2 & 3 \\
\hline
\end{tabular}




\section{Organization}

Univ Muhammadiyah Palangkaraya

Univ Sao Paulo

Uri Erechim

Urmia Univ Med Sci

Acad Ivodent

Alkarkh Univ Sci

Alten

Bindura Univ Sci Educ

Biotechnol \& Bioengn Res \& Applicat Ctr Biomer

Carmat Sa

Cemes Cnrs

Commerce Minist

Ctr Ensino Riograndense

Cukurova Univ

D Moulay Taher Univ

Di Mendeleev Univ Chem Technol Russia Muctr

Durban Univ Technol

Fac Med \& Biomed Sci

Giresun Univ

Ibn Khaldoun Univ

Ibn Khaldoun Univ Tiaret

Imam Mohammad Ibn Saud Islamic Univ

Innovatecs Biotechnol Res \& Dev

Inoprod

Izmir Inst Technol

Kalasalingam Univ

Malek Ashtar Univ

Manicaland State Univ Appl Sci

Manisa Celal Bayar Univ

Mawlana Bhashani Sci \& Technol Univ Santosh

Midlands State Univ

Minist Justice

Minist Women \& Children Affairs

Mother Theresa Coll Pharm

Niist

Nile Univ

Normandie Univ

Oranl Univ Ahmed Benbella

Port Said Univ

Rajeev Gandhi Mem Coll Engn \& Technol

Rangamati Sci \& Technol Univ

Tomsk State Univ

Trisakti Univ

Univ Babylon

Univ Monastir

Univ Prof Dr Asen Zlatarov

Univ Siena

Univ Tirana

Vr Coll

William Osler Hlth Ctr

Yogi Vemana Univ

Inst Politecn Nacl

Galgotias Univ

Univ Buenos Aires

Donbass State Engn Acad

Lviv Natl Polytech Univ

Mahsa Univ

Mgr Coll

Natl Inst Res \& Dev Environm Protect

Natl Res Inst Astron \& Geophys Nano Nriag

Univ Milan

Annamalai Univ

Banaras Hindu Univ

Hazara Univ

Imperial Coll

Kemerovo State Univ

\begin{tabular}{|l|l} 
Documents & Citations \\
\hline
\end{tabular}

2

3

\section{3}

\begin{tabular}{|l|l|}
\hline 2 & 3 \\
\hline 2 & 3 \\
\hline
\end{tabular}

2

1

1

1
1
1

1

1

1

1

1

1

1

1

1

1
1
1

1

1

1

1

1

1

1

1

1
1
1

1

1
1
1

1
1
1

1

1
1
1

\begin{tabular}{|l}
1 \\
\hline 1 \\
\hline 1
\end{tabular}

\begin{tabular}{l}
1 \\
1 \\
\hline
\end{tabular}

1

1

15

\begin{tabular}{|l|l}
4 \\
4 \\
3
\end{tabular}

\begin{tabular}{l}
3 \\
3 \\
\hline
\end{tabular}

\begin{tabular}{l}
3 \\
3 \\
\hline
\end{tabular}

\begin{tabular}{l}
3 \\
3 \\
\hline
\end{tabular}

\begin{tabular}{l}
3 \\
3 \\
\hline
\end{tabular}

3

3

\begin{tabular}{l}
2 \\
2 \\
\hline
\end{tabular}

2

2

2

\begin{tabular}{l}
3 \\
3 \\
\hline
\end{tabular}

(2)

\begin{tabular}{l|l}
\hline & 3 \\
\hline & 3 \\
\hline
\end{tabular}

3
3
3
3
3
3

3

3
3
3
3

3

3

3

3

3

3

\begin{tabular}{l}
3 \\
3 \\
\hline
\end{tabular}

3
3
3
3

$\frac{3}{3}$

3

\begin{tabular}{l}
3 \\
\hline 3 \\
\hline 3 \\
\hline 3 \\
\hline 2 \\
\hline 2 \\
\hline 2 \\
\hline 2 \\
\hline 2 \\
\hline 2 \\
\hline 2 \\
\hline 2 \\
\hline 2 \\
\hline 2 \\
\hline 2 \\
\hline 2 \\
\hline 2 \\
\hline 2
\end{tabular}


Organization

Rama Devi Womens Univ

Semnan Univ Med Sci

Univ Agr Faisalabad

Univ Food Technol

Univ Kristen Indonesia Toraja

Univ Nacl Autonoma Mexico

Ab Sciex Thailand Ltd

Adiyaman Univ

Agricultural Acad

Anshun Univ

Antalya Bilim Univ

Aristotle Univ Thessaloniki

Azienda Us1 9 Grosseto

Belarusian State Univ

Brawijaya Univ

Bulent Ecevit Univ

Christ Univ

Djilaliliabesuniversty

Dr Dy Patil Univ

Drexel Univ

Fac Med Biosci \& Nursing

Fac Sci Tunis

Fed Univ Santa Catarina Ufsc

Fed Univ Sao Paulo Unifesp

Gadjah Mada Univ

Grad Univ Adv Technol

Guizhou Univ

Hakim Sabzevari Univ

Hanoi Univ Ind

Higher Inst Technol \& Appl Sci Instec

Ibnkhaldoun Univ Tiaret

Icfai Univ

Ifremer

Im Sechenov First Moscow State Med Univ

Inst Chem Vast

Inst Natl Sci \& Technol Mer

Istanbul Arel Univ Turkey

Kafrelsheikh Univ

Kamaraj Coll Arts \& Sci

Kharkiv Natl Med Univ

King Mongkuts Univ Technol Thonburi

Kmt Primary Care Ctr

Mohi Ud Din Islamic Univ

Nanjing Agr Univ

Natl Cent Univ

Natl Res Inst Astron \& Geophys

Natl Univ Pharm

Noida Inst Engn \& Technol

Nusa Nipa Univ

Oleksandr Dovzhenko Hlukhiv Natl Pedag Univ

Phranakhon Rajabhat Univ

Pt Jawaharlal Nehru Coll

Pukyong Natl Univ

Sekolah Progresif Bumi Shalawat

Siksha O Anusandhan

Srinakharinwirot Univ

St Petersburg State Polytech Univ

Stand Res Org

Ucsi Univ

Univ Abdelamlek Essaadi

Univ Extremadura

Univ Johannesburg

Univ Maragheh

Univ Sumatera Utara

Virginia Tech

Yanka Kupala State Univ Grodno

\begin{tabular}{|l|l} 
Documents & Citations \\
\hline
\end{tabular}

2

2

\begin{tabular}{l|l}
2 & 2 \\
\hline
\end{tabular}

\begin{tabular}{l|l}
2 & 2 \\
2 & 2
\end{tabular}

\begin{tabular}{l|l}
2 & 2 \\
\hline 2 & 2 \\
\hline
\end{tabular}

2

1

1

1

1

\begin{tabular}{l}
1 \\
1 \\
\hline
\end{tabular}

1

1

1

1
1

1

1
1
1

1

1

1
1
1

1

1
1
1
1

1

1

1

1

1
1
1

1

1
1
1

\begin{tabular}{l}
1 \\
1 \\
1 \\
\hline
\end{tabular}

1

1
1
1

1
1
1

1

1
1
1

1

1
1
1

1

1
1
1

1
1
1
1

1
1
1

1
1
1
1

1
1
1
1

1

1

2

2

2
2
2
2
2
2

2

2

2

2

2

2

2
2
2
2

2
2
2
2

2
2
2
2
2

2

2
2
2




\begin{tabular}{|c|c|c|}
\hline Organization & Documents & Citations \\
\hline Yarmouk Univ & 1 & 2 \\
\hline Yazd Univ & 1 & 2 \\
\hline Yildiz Tech Univ & 1 & 2 \\
\hline I Horbachevsky Ternopil Natl Med Univ & 5 & 1 \\
\hline Bukovinian State Med Univ & 4 & 1 \\
\hline Giet Univ & 4 & 1 \\
\hline Univ Autonoma Carmen & 4 & 1 \\
\hline Univ Med \& Pharm Carol Davila & 4 & 1 \\
\hline Amity Univ & 3 & 1 \\
\hline Assoc Epigenet \& Metabol & 3 & 1 \\
\hline Cent Reference Lab Synevo & 3 & 1 \\
\hline Chitkara Univ & 3 & 1 \\
\hline Inst Minerals \& Mat Technol Csir & 3 & 1 \\
\hline Lovely Profess Univ & 3 & 1 \\
\hline Soa Deemed Be Univ & 3 & 1 \\
\hline Tarbiat Modares Univ & 3 & 1 \\
\hline Univ Fed Santa Maria & 3 & 1 \\
\hline Univ Fed Vicosa & 3 & 1 \\
\hline Univ Halu Oleo & 3 & 1 \\
\hline Aja Univ Med Sci & 2 & 1 \\
\hline Assiut Univ & 2 & 1 \\
\hline Bogor Agr Univ & 2 & 1 \\
\hline Chinese Acad Sci & 2 & 1 \\
\hline Imam Mohammad Ibn Saud Islamic Univ Imsiu & 2 & 1 \\
\hline Indian Inst Chem Technol & 2 & 1 \\
\hline Kazan Fed Univ & 2 & 1 \\
\hline Koneru Lakshmaiah Educ Fdn & 2 & 1 \\
\hline Natl Inst Technol & 2 & 1 \\
\hline Nigde Omer Halisdemir Univ & 2 & 1 \\
\hline Reg Inst Educ Ncert & 2 & 1 \\
\hline Sekolah Tinggi Farmasi Indonesia & 2 & 1 \\
\hline Semnan Univ & 2 & 1 \\
\hline Univ Kebangsaan Malaysia & 2 & 1 \\
\hline Univ Malaya & 2 & 1 \\
\hline Univ Manchester & 2 & 1 \\
\hline Visva Bharati Univ & 2 & 1 \\
\hline 8054 Monash Univ Lpo & 1 & 1 \\
\hline Abdulwali Khan Univ & 1 & 1 \\
\hline Aksaray Univ & 1 & 1 \\
\hline Alanya Alaaddin Keykubat Univ & 1 & 1 \\
\hline All St Univ & 1 & 1 \\
\hline Allama Iqbal Open Univ & 1 & 1 \\
\hline Ardabil Univ Med Sci & 1 & 1 \\
\hline Assoc Educ Nutr & 1 & 1 \\
\hline Atom Energy Ctr & 1 & 1 \\
\hline Balaji Inst Pharmaceut Sci & 1 & 1 \\
\hline Belgorod State Technol Univ & 1 & 1 \\
\hline Biotechnol Ctr Borj Cedria Technopk & 1 & 1 \\
\hline Cairo Higher Inst Engn & 1 & 1 \\
\hline Catholic Univ & 1 & 1 \\
\hline Centurion Univ & 1 & 1 \\
\hline Chernivtsi Natl Univ & 1 & 1 \\
\hline Chinese Acad Agr Sci & 1 & 1 \\
\hline $\mathrm{Cnr}$ & 1 & 1 \\
\hline Comsats Inst Infonnat Technol & 1 & 1 \\
\hline Ctr Phys Sci \& Technol & 1 & 1 \\
\hline Ctr Rech Interdisciplinaire Readaptat Montreal Me & 1 & 1 \\
\hline Ctr Text & 1 & 1 \\
\hline Damanhour Univ & 1 & 1 \\
\hline Danylo Halytsky Lviv Natl Med Univ Lviv & 1 & 1 \\
\hline Dayananda Sagar Acad Technol \& Management & 1 & 1 \\
\hline Dde Annamalai Univ & 1 & 1 \\
\hline Delta Univ & 1 & 1 \\
\hline Dent Estetika Ltd & 1 & 1 \\
\hline Djilali Liabesuniversty & 1 & 1 \\
\hline Dnipropetrovsk State Med Acad & 1 & 1 \\
\hline
\end{tabular}




\begin{tabular}{|c|c|c|}
\hline Organization & Documents & Citations \\
\hline Donbas State Engn Acad & 1 & 1 \\
\hline E China Univ Sci \& Technol & 1 & 1 \\
\hline Ecopara Diag Med Ctr & 1 & 1 \\
\hline Environm Monitoring Ctr Taizhou & 1 & 1 \\
\hline Fac Ciencias Exactas Unlp & 1 & 1 \\
\hline Fda & 1 & 1 \\
\hline Fed Med Ctr & 1 & 1 \\
\hline Fed Univ Juiz De For Governador Valadares & 1 & 1 \\
\hline Fed Univ Para & 1 & 1 \\
\hline Fed Univ Rio Gande Do Sul Ufrgs & 1 & 1 \\
\hline Fed Univ South Mato Grosso & 1 & 1 \\
\hline Fundacao Univ Fed Rio Grande & 1 & 1 \\
\hline Gaziantep Univ & 1 & 1 \\
\hline Gbpuat & 1 & 1 \\
\hline Gharda Inst Technol & 1 & 1 \\
\hline Govt Sci Coll & 1 & 1 \\
\hline Harkiv Natl Med Univ & 1 & 1 \\
\hline Hebei Univ Technol & 1 & 1 \\
\hline Horbachevsky Ternopil Natl Med Univ Ternopil & 1 & 1 \\
\hline Horus Univ & 1 & 1 \\
\hline Hosp Reghaia & 1 & 1 \\
\hline Housing \& Bldg Natl Res Ctr Hbrc & 1 & 1 \\
\hline I Horbachevsky Ternopil Natl Med Univ Ternopil & 1 & 1 \\
\hline I Horbachevsky Ternopil State Med Univ & 1 & 1 \\
\hline Ibn Tofail Univ & 1 & 1 \\
\hline Ibrahim Badamasi Babangida Univ & 1 & 1 \\
\hline Independent Univ & 1 & 1 \\
\hline Indian Inst Sci & 1 & 1 \\
\hline Indonesian Inst Sci & 1 & 1 \\
\hline Inst Fed Educ Ciencia \& Tecnol Rio De Janeiro Ifr & 1 & 1 \\
\hline Inst Fed Parana Ifpr & 1 & 1 \\
\hline Iran Polymer \& Petrochem Inst & 1 & 1 \\
\hline Iranian Natl Stand Org & 1 & 1 \\
\hline Irri & 1 & 1 \\
\hline Itm Univ & 1 & 1 \\
\hline Iy Horbachevsky State Med Univ & 1 & 1 \\
\hline Jahangirnagar Univ & 1 & 1 \\
\hline Jamia Hamdard & 1 & 1 \\
\hline Jiangsu Key Lab Precious Met Chem \& Engn & 1 & 1 \\
\hline Jiangsu Univ Technol & 1 & 1 \\
\hline Jipmer & 1 & 1 \\
\hline Karunya Inst Sci \& Technol & 1 & 1 \\
\hline Katholieke Univ Leuven & 1 & 1 \\
\hline Kazan Natl Res Technol Univ & 1 & 1 \\
\hline Kerman Momtazan Cement Co & 1 & 1 \\
\hline King Abdul Aziz City Sci \& Technol & 1 & 1 \\
\hline Kle Univ & 1 & 1 \\
\hline Kspoau Kamchatka Polytech Coll & 1 & 1 \\
\hline Kumaraguru Coll Technol & 1 & 1 \\
\hline Lady Reading Hosp Peshawar & 1 & 1 \\
\hline Lahore Coll Women Univ & 1 & 1 \\
\hline Leibniz Inst Polymerforsch Dresden Ev & 1 & 1 \\
\hline Madanapalle Inst Technol \& Sci & 1 & 1 \\
\hline Marrilaxm Reddy Inst Technol \& Management & 1 & 1 \\
\hline Masaryk Univ & 1 & 1 \\
\hline Mat \& Energy Res Ctr & 1 & 1 \\
\hline Math Lax Man Reddy Inst Technol \& Management & 1 & 1 \\
\hline Minist Antiqu & 1 & 1 \\
\hline Minist Hlth Russian Federat & 1 & 1 \\
\hline Minist Saude & 1 & 1 \\
\hline Misan Univ & 1 & 1 \\
\hline Motherhood Univ & 1 & 1 \\
\hline Mugla Sitki Koyman Univ & 1 & 1 \\
\hline Nams Ukraina & 1 & 1 \\
\hline Natl Canc Inst & 1 & 1 \\
\hline Natl Inst Virol & 1 & 1 \\
\hline
\end{tabular}


Organization

Natl Tech Univ Athens

Natl Univ Salta

Natl Univ Singapore Nus

Ncsr Demokritos

Northeast Normal Univ

Northern Border Univ Rafha

Novie Nauchnie Technol Ltd

Plekhanov Russian Univ Econ

Prevenlab Lab

Pushpagiri Coll Pharm

Qurtaba Univ

Replek Farm Ltd

Res Inst Ophthalmol

Romanian Acad

Sam Higginbotton Univ Agr Technol \& Sci

Sheda Sci \& Technol Complex

Singapore Eye Res Inst Seri

South Ural State Univ

Southwest State Univ

Sree Narayana Coll

Suleyman Demirel Univ

Sunyatsen Univ

Synevo Grozovici

Taif Univ

Tech Univ Denmark

Ternopil Reg Drug Treatment Clin

Tf Gorbachev Kuzbass State Tech Univ Kuzstu

Titu Maiorescu Univ Bucharest

Tripura Univ

Univ Alzahra

Univ Artois

Univ Autonama Estado Morelos

Univ Brasilia

Univ Cent Ecuador

Univ Clin Fundeni

Univ Ctr Ain Temouchent

Univ Dhaka

Univ Djilali Liabes

Univ Fed Ceara

Univ Fed Goias

Univ Guilan

Univ Hassiba Benbouali Chlef

Univ Ibn Khaldoun

Univ Ibn Khaldoun Tiaret

Univ Ilorin

Univ Malakand

Univ Nevada

Univ Oran Ahmed Ben Bella

Univ Oslo

Univ Politecn Madrid

Univ Putra Malaysia

Univ Sci \& Technol Usthb

Univ Sidi Mohammed Ben Abdellah

Univ Texas Austin

Univ Toronto

Univ Vet \& Pharmaceut Sci Brno

Vet Res \& Diagnost Lab

Vfstr Deemed Be Univ

Xuchang Univ

Yanbu Ind Coll

Yildiz Tekn Univ

Zaporizhzhya Med Acad Postgrad Educ

Zhejiang Univ

Vikram Deb Autonomous Coll

Orenburg State Univ

Siksha O Anusandhan Deemed Univ

\begin{tabular}{|l|l} 
Documents & Citations \\
\hline
\end{tabular}

\begin{tabular}{l|l}
1 & 1 \\
\hline 1 & 1 \\
\hline 1 & 1 \\
\hline
\end{tabular}

1

1

1

1

1

1

1

1

1

1

1

1

1

1

1

1

1

1

1

1

1

\begin{tabular}{l}
1 \\
1 \\
\hline
\end{tabular}

1

1
1
1

1

1
1
1

\begin{tabular}{l}
1 \\
1 \\
1 \\
\hline
\end{tabular}

1

\begin{tabular}{l}
1 \\
1 \\
\hline
\end{tabular}

\begin{tabular}{l}
1 \\
1 \\
1 \\
\hline
\end{tabular}

1

1

1

\begin{tabular}{l}
1 \\
1 \\
\hline
\end{tabular}

1

1
1
1

1

1

1

1
1
1

1

1
1
1

1
1
5

5

\begin{tabular}{|l|l|}
\hline & 5 \\
3
\end{tabular}

4 
Organization

Univ Juarez Estado Durango

Univ Politehn Bucuresti

Univ Tun Hussein Onn Malaysia

Baku State Univ

Bu Ali Sina Univ

Colentina Clin Hosp

Csir Niist

El Mansoura Univ

Fundeni Clin Inst

Gubkin Univ

Hefei Gas Grp

Hefei Univ

Higher Inst Engn \& Technol El Arish

Iancului Private Lab

Isfahan Univ Med Sci

Jingdezhen Ceram Inst

Lomonosov Moscow State Univ

Mekelle Univ

Nanyang Technol Univ

Ocean Univ China

Qingdao Natl Lab Marine Sci \& Technol

Res Ctr Studies Food Qual \& Agr Prod

Sd Coll Pharm \& Vocat Studies

Shorter Univ

Southern Polytech State Univ

Titu Maiorescu Univ

Univ Basrah

Univ Rhode Isl

Univ Rzeszow

Univ Sci \& Technol China

Univ Seville

Univ Teknol Mara

Victor Babes Med \& Pharmaceut Univ Timisoara

Vishveshwarya Grp Inst

Yunnan Agr Univ

Abdul Wali Khan Univ Mardan Khyber Pakhtunkhwa

Abdul Wali Khan Univ Mardan Kp

Acad Romanian Scientists

Adelaide Univ

Aditya Coll Engn

Agensi Nuklear Malaysia

Aievol Consulting Ltd

Air Univ

Al Qadisiyah Univ

All Russian Res Inst Phytopathol

Alma Mater Studiorum Univ Bologna

Alma Mater Studiorum Univ Bologna Ozzano Emilia

Amirkabir Univ

Andhra Univ

Anjuman I Islams Kalsekar Tech Campus

Assam Agr Univ Aau Jorhat

Azerbaijan Med Univ

Azerbaijan Natl Acad Sci

Azerbaijan State Pedag Univ

Babol Noshirvani Univ Technol

Babol Univ Med Sci

Badji Mokhtar Univ

Balasagyn Kyrgyz Natl Univ

Bankura Sammilani Coll

Bar Ilan Univ

Bartin Univ

Bashkir State Univ

Belgorod State Univ

Bharathidasan Univ

Bio \& Nanosolut

Biochem

\begin{tabular}{|c|c|}
\hline Documents & Citations \\
\hline 3 & 0 \\
\hline 3 & 0 \\
\hline 3 & 0 \\
\hline 2 & 0 \\
\hline 2 & 0 \\
\hline 2 & 0 \\
\hline 2 & 0 \\
\hline 2 & 0 \\
\hline 2 & 0 \\
\hline 2 & 0 \\
\hline 2 & 0 \\
\hline 2 & 0 \\
\hline 2 & 0 \\
\hline 2 & 0 \\
\hline 2 & 0 \\
\hline 2 & 0 \\
\hline 2 & 0 \\
\hline 2 & 0 \\
\hline 2 & 0 \\
\hline 2 & 0 \\
\hline 2 & 0 \\
\hline 2 & 0 \\
\hline 2 & 0 \\
\hline 2 & 0 \\
\hline 2 & 0 \\
\hline 2 & 0 \\
\hline 2 & 0 \\
\hline 2 & 0 \\
\hline 2 & 0 \\
\hline 2 & 0 \\
\hline 2 & 0 \\
\hline 2 & 0 \\
\hline 2 & 0 \\
\hline 2 & 0 \\
\hline 2 & 0 \\
\hline 1 & 0 \\
\hline 1 & 0 \\
\hline 1 & 0 \\
\hline 1 & 0 \\
\hline 1 & 0 \\
\hline 1 & 0 \\
\hline 1 & 0 \\
\hline 1 & 0 \\
\hline 1 & 0 \\
\hline 1 & 0 \\
\hline 1 & 0 \\
\hline 1 & 0 \\
\hline 1 & 0 \\
\hline 1 & 0 \\
\hline 1 & 0 \\
\hline 1 & 0 \\
\hline 1 & 0 \\
\hline 1 & 0 \\
\hline 1 & 0 \\
\hline 1 & 0 \\
\hline 1 & 0 \\
\hline 1 & 0 \\
\hline 1 & 0 \\
\hline 1 & 0 \\
\hline 1 & 0 \\
\hline 1 & 0 \\
\hline 1 & 0 \\
\hline 1 & 0 \\
\hline 1 & 0 \\
\hline 1 & 0 \\
\hline 1 & 0 \\
\hline
\end{tabular}




\begin{tabular}{|c|c|c|}
\hline Organization & Documents & Citations \\
\hline Biochem Engn Mat & 1 & 0 \\
\hline Bit Mesra & 1 & 0 \\
\hline Bue & 1 & 0 \\
\hline Ca Foscari Univ Venice & 1 & 0 \\
\hline Carol Davila Med \& Pharmaceut Univ & 1 & 0 \\
\hline Cas & 1 & 0 \\
\hline Catholic Univ San Antonio Murcia Ucam & 1 & 0 \\
\hline Cent Referencelaboratorysynevo & 1 & 0 \\
\hline Cent Univ Gujarat & 1 & 0 \\
\hline Cent Univ Technol & 1 & 0 \\
\hline Centurion Univ Technol \& Management & 1 & 0 \\
\hline Chelyabinsk State Univ & 1 & 0 \\
\hline Clin Inst Fundeni & 1 & 0 \\
\hline Cmr Inst Technol & 1 & 0 \\
\hline Cnrs Um Um Enscm & 1 & 0 \\
\hline Consortium Instm Ru Venice & 1 & 0 \\
\hline Csir Cent Salt \& Marine Chem Res Inst & 1 & 0 \\
\hline Csir North East Inst Sci \& Technol Neist & 1 & 0 \\
\hline Ctr Drug Res \& Dev & 1 & 0 \\
\hline Ctr Engn \& Chem Invest & 1 & 0 \\
\hline Cyberjaya Univ & 1 & 0 \\
\hline Damietta Univ & 1 & 0 \\
\hline Dayanand Sagar Univ & 1 & 0 \\
\hline Deogiri Coll & 1 & 0 \\
\hline Dharmsinh Desai Univ & 1 & 0 \\
\hline Dnipro Univ Technol & 1 & 0 \\
\hline Dr Soetomo Univ & 1 & 0 \\
\hline Dubai Hlth Author & 1 & 0 \\
\hline Escuela Nacl Ciencias Biol & 1 & 0 \\
\hline European Commiss & 1 & 0 \\
\hline Fac Concordia Facc & 1 & 0 \\
\hline Fed Univ Santa Maria Ufsm & 1 & 0 \\
\hline Fic Kazansc Ras & 1 & 0 \\
\hline Future Univ & 1 & 0 \\
\hline Galgot Univ & 1 & 0 \\
\hline Galgotia Univ & 1 & 0 \\
\hline Gazi Univ & 1 & 0 \\
\hline Gaziosmanpasa Univ & 1 & 0 \\
\hline Govt Punjab & 1 & 0 \\
\hline Guru Jambheshwar Univ Sci \& Technol & 1 & 0 \\
\hline Gyeongsang Natl Univ & 1 & 0 \\
\hline Hemchandracharya North Gujarat Univ & 1 & 0 \\
\hline Higher State Educ Estab Bukovinian State Med Univ & 1 & 0 \\
\hline Holon Inst Technol & 1 & 0 \\
\hline Ibb Univ & 1 & 0 \\
\hline Imsiu & 1 & 0 \\
\hline Indian Council Med Res & 1 & 0 \\
\hline Indian Inst Technol Delhi & 1 & 0 \\
\hline Indian Inst Technol Roorkee & 1 & 0 \\
\hline Indian Sch Mines & 1 & 0 \\
\hline Indonesia Int Inst Life Sci I3l & 1 & 0 \\
\hline Inst Chem \& Chem Technol & 1 & 0 \\
\hline Inst Fed Educ Ciencia \& Tecnol Maranhao & 1 & 0 \\
\hline Inst Publ Hlth & 1 & 0 \\
\hline Inst Virol St Nicolau & 1 & 0 \\
\hline Iran Univ Sci \& Technol & 1 & 0 \\
\hline Irccs Ist Ortoped Rizzoli & 1 & 0 \\
\hline Islamic Azad Univ Sabzevar Ium & 1 & 0 \\
\hline Islamic Azad Univ Tehran Ium & 1 & 0 \\
\hline Jawaharlal Nehru Technol Univ & 1 & 0 \\
\hline Jeonju Univ & 1 & 0 \\
\hline Jntu & 1 & 0 \\
\hline Kabul Univ & 1 & 0 \\
\hline Karadeniz Tech Univ & 1 & 0 \\
\hline Khabarovsk State Univ Econ \& Law & 1 & 0 \\
\hline Kharkiv Med Acad Postgrad Educ & 1 & 0 \\
\hline
\end{tabular}




\begin{tabular}{|c|c|c|}
\hline Organization & Documents & Citations \\
\hline Kharkov Natl Univ & 1 & 0 \\
\hline Kiit Univ & 1 & 0 \\
\hline Kohat Univ Sci \& Technol Kohat & 1 & 0 \\
\hline Kurukshetra Univ & 1 & 0 \\
\hline Kuzbass State Tech Univ & 1 & 0 \\
\hline Lasbela Univ Agr Water \& Marine Sci & 1 & 0 \\
\hline Latvia Univ Life Sci \& Technol & 1 & 0 \\
\hline Liaoning Univ & 1 & 0 \\
\hline Maharishi Markandeshwar Univ & 1 & 0 \\
\hline Maharshi Dayanand Univ & 1 & 0 \\
\hline Malayer Univ & 1 & 0 \\
\hline Maratha Mandal Res Ctr & 1 & 0 \\
\hline Maulana Azad Natl Inst Technol & 1 & 0 \\
\hline Med Res Ctr & 1 & 0 \\
\hline Med Univ & 1 & 0 \\
\hline Medlife & 1 & 0 \\
\hline Mendeleev Univ Chem Technol Russia & 1 & 0 \\
\hline Mendeleev Univ Chem Technol Russia Muctr & 1 & 0 \\
\hline Meram Training \& Res Hosp & 1 & 0 \\
\hline Misr Int Univ & 1 & 0 \\
\hline Mohammed V Univ & 1 & 0 \\
\hline Mohammed V Univ Rabat & 1 & 0 \\
\hline Moulay Ismail Univ & 1 & 0 \\
\hline Must & 1 & 0 \\
\hline Mwenge Catholic Univ & 1 & 0 \\
\hline Mysuru & 1 & 0 \\
\hline Namik Kemal Univ & 1 & 0 \\
\hline Narc & 1 & 0 \\
\hline Natl \& Kapodistrian Univ Athens & 1 & 0 \\
\hline Natl Inst Res \& Dev Environm Protect Bucharest & 1 & 0 \\
\hline Natl Inst Res Cantacuzino & 1 & 0 \\
\hline Nelson Mandela African Inst Sci \& Technol & 1 & 0 \\
\hline North Fluminense State Univ & 1 & 0 \\
\hline Nursing Sch Al Anbar Directorate Hlth & 1 & 0 \\
\hline Okan Univ & 1 & 0 \\
\hline Orenburg State Agr Univ & 1 & 0 \\
\hline Orissa Univ Agr \& Technol & 1 & 0 \\
\hline Ouat & 1 & 0 \\
\hline Ovidius Univ & 1 & 0 \\
\hline Pacific Natl Univ & 1 & 0 \\
\hline Piramal Enterprises Limited & 1 & 0 \\
\hline Pmas Arid Agr Univ & 1 & 0 \\
\hline Pontificia Univ Catolica Rio De Janeiro & 1 & 0 \\
\hline Pooja Bhagavat Mem Mahajana Pg Ctr & 1 & 0 \\
\hline Rajiv Gandhi Proudyogiki Vishwavidyalaya & 1 & 0 \\
\hline Ras & 1 & 0 \\
\hline Res Ctr Biomat & 1 & 0 \\
\hline Res Ctr Chem & 1 & 0 \\
\hline Res Inst Petr Ind & 1 & 0 \\
\hline Rv Engn Coll & 1 & 0 \\
\hline Ryerson Univ & 1 & 0 \\
\hline Sanaa Univ & 1 & 0 \\
\hline Sao Paulo State Univ Unesp & 1 & 0 \\
\hline Sekolah Tinggi Ilmu Farmasi Makassar & 1 & 0 \\
\hline Shahid Bahonar Univ & 1 & 0 \\
\hline Shahrekord Univ & 1 & 0 \\
\hline Shahrekord Univ Med Sci & 1 & 0 \\
\hline Shandong Inst Pharmaceut Ind & 1 & 0 \\
\hline Shanghai Jiao Tong Univ & 1 & 0 \\
\hline Shanghai Univ & 1 & 0 \\
\hline Shaqra Univ & 1 & 0 \\
\hline Sharjah Res Acad & 1 & 0 \\
\hline Sichuan Univ & 1 & 0 \\
\hline Siksha O Anusandhana Deemed Be Univ & 1 & 0 \\
\hline Silicon Inst Technol & 1 & 0 \\
\hline Soa Univ & 1 & 0 \\
\hline
\end{tabular}




\begin{tabular}{|c|c|c|}
\hline Organization & Documents & Citations \\
\hline Sooft Italia Spa & 1 & 0 \\
\hline South China Agr Univ & 1 & 0 \\
\hline St Anna Hosp & 1 & 0 \\
\hline St Orsola Marcello Malpighi Hosp & 1 & 0 \\
\hline State Univ Land Use Planning & 1 & 0 \\
\hline Stefan S Nicolau Inst Virol & 1 & 0 \\
\hline Suez Canal Univ & 1 & 0 \\
\hline Sumgait State Univ & 1 & 0 \\
\hline Svkms Nmims & 1 & 0 \\
\hline Svs Coll Pharmaceut Sci & 1 & 0 \\
\hline Taxon Solut & 1 & 0 \\
\hline Tech Univ Carolo Wilhelmina Braunschweig & 1 & 0 \\
\hline Tech Univ Gabrovo & 1 & 0 \\
\hline Telecommun \& Mat Sci Engn Lab Locem & 1 & 0 \\
\hline Thirukollanjiappar Arts Coll & 1 & 0 \\
\hline Tofigh Daru Res \& Engn Co & 1 & 0 \\
\hline Tokyo Univ Sci & 1 & 0 \\
\hline Tomas Bata Univ Zlin & 1 & 0 \\
\hline Tribhuvan Univ & 1 & 0 \\
\hline Trivedi Global Inc & 1 & 0 \\
\hline Trivedi Sci Res Lab Pvt Ltd & 1 & 0 \\
\hline Uenf & 1 & 0 \\
\hline Ufa State Petr Tech Univ & 1 & 0 \\
\hline Ukrainian State Chem Technol Univ & 1 & 0 \\
\hline Umm Al Qura Univ & 1 & 0 \\
\hline Unist & 1 & 0 \\
\hline Univ Alcala De Henares & 1 & 0 \\
\hline Univ Anbar & 1 & 0 \\
\hline Univ Birmingham & 1 & 0 \\
\hline Univ British Columbia & 1 & 0 \\
\hline Univ Coll Technol & 1 & 0 \\
\hline Univ Contestado & 1 & 0 \\
\hline Univ Djillali Liabes Sidi Belabbes & 1 & 0 \\
\hline Univ Educ Lahore & 1 & 0 \\
\hline Univ Eia & 1 & 0 \\
\hline Univ Elect Sci \& Technol China & 1 & 0 \\
\hline Univ Engn \& Management & 1 & 0 \\
\hline Univ Fed Fluminense & 1 & 0 \\
\hline Univ Fed Maranhao & 1 & 0 \\
\hline Univ Fed Rio Grande Do Norte & 1 & 0 \\
\hline Univ Fed Uberlandia & 1 & 0 \\
\hline Univ Ferrara & 1 & 0 \\
\hline Univ Freiburg & 1 & 0 \\
\hline Univ G Dannunzio & 1 & 0 \\
\hline Univ Ghana & 1 & 0 \\
\hline Univ Iben Khaldoun & 1 & 0 \\
\hline Univ Icesi & 1 & 0 \\
\hline Univ Juarez Durango & 1 & 0 \\
\hline Univ Kingston & 1 & 0 \\
\hline Univ Leicester & 1 & 0 \\
\hline Univ Los Andes & 1 & 0 \\
\hline Univ Lucknow & 1 & 0 \\
\hline Univ Malaysia Sarawak & 1 & 0 \\
\hline Univ Manuela Beltran & 1 & 0 \\
\hline Univ Medea & 1 & 0 \\
\hline Univ Moratuwa & 1 & 0 \\
\hline Univ Mumbai & 1 & 0 \\
\hline Univ Potiguar Laureate Int Univ & 1 & 0 \\
\hline Univ Rennes 1 & 1 & 0 \\
\hline Univ Rouen & 1 & 0 \\
\hline Univ Salerno & 1 & 0 \\
\hline Univ Sharjah & 1 & 0 \\
\hline Univ Sopron & 1 & 0 \\
\hline Univ Tunis El Manar & 1 & 0 \\
\hline Univ Vet \& Anim Sci & 1 & 0 \\
\hline Univ Zagreb & 1 & 0 \\
\hline
\end{tabular}




\begin{tabular}{l|l|l} 
Organization & Documents & Citations \\
\hline Veer Surendra Sai Univ Technol & 1 & 0 \\
\hline Vel Tech Rangarajan Dr Sagunthala R\&D Inst Sci \& & 1 & 0 \\
\hline Vet Res Inst & 1 & 0 \\
\hline Via Vestini 31 & 1 & 0 \\
\hline Victor Segalen Univ Bordeaux2 & 1 & 0 \\
\hline Vignans Fdn Sci Technol \& Res & 1 & 0 \\
\hline Villaserena Fdn Res & 1 & 0 \\
\hline Yeltsin Ural Fed Univ & 1 & 0 \\
\hline Zanjan Univ Med Sci & 1 & 0 \\
\hline Zaporizhzhia State Med Univ & 1 & 0 \\
\hline Zhytomyr Ivan Franko State Univ & 1 & 0
\end{tabular}

Table S3. List of 77 countries with total documents and citations.

\begin{tabular}{|c|c|c|}
\hline Country & Documents & Citations \\
\hline Iran & 164 & 412 \\
\hline India & 152 & 281 \\
\hline Egypt & 72 & 223 \\
\hline Saudi Arabia & 32 & 82 \\
\hline Brazil & 35 & 73 \\
\hline Russia & 39 & 62 \\
\hline Malaysia & 37 & 61 \\
\hline Romania & 35 & 55 \\
\hline Turkey & 33 & 52 \\
\hline Indonesia & 29 & 49 \\
\hline Pakistan & 24 & 36 \\
\hline USA & 16 & 29 \\
\hline Vietnam & 11 & 29 \\
\hline Ukraine & 23 & 27 \\
\hline Taiwan & 3 & 26 \\
\hline Italy & 16 & 22 \\
\hline Argentina & 12 & 19 \\
\hline Peoples R China & 25 & 17 \\
\hline Algeria & 12 & 15 \\
\hline Bulgaria & 5 & 15 \\
\hline France & 8 & 13 \\
\hline Spain & 7 & 13 \\
\hline Thailand & 7 & 13 \\
\hline Mexico & 21 & 10 \\
\hline Canada & 6 & 10 \\
\hline Scotland & 2 & 10 \\
\hline Belgium & 2 & 9 \\
\hline Germany & 7 & 8 \\
\hline Bangladesh & 5 & 8 \\
\hline Australia & 4 & 8 \\
\hline Czech Republic & 4 & 8 \\
\hline Austria & 1 & 8 \\
\hline Nigeria & 2 & 7 \\
\hline Chile & 1 & 7 \\
\hline South Korea & 4 & 6 \\
\hline Tunisia & 4 & 6 \\
\hline Denmark & 2 & 6 \\
\hline Dominica & 1 & 6 \\
\hline Cuba & 4 & 5 \\
\hline Jordan & 3 & 5 \\
\hline South Africa & 3 & 5 \\
\hline Sudan & 1 & 5 \\
\hline Iraq & 5 & 4 \\
\hline Oman & 2 & 4 \\
\hline Brunei & 1 & 4 \\
\hline Wales & 1 & 4 \\
\hline England & 8 & 3 \\
\hline Morocco & 5 & 3 \\
\hline Greece & 3 & 3 \\
\hline Albania & 1 & 3 \\
\hline Zimbabwe & 1 & 3 \\
\hline Belarus & 1 & 2 \\
\hline
\end{tabular}




\begin{tabular}{l|l|l} 
Country & Documents & Citations \\
\hline Singapore & 3 & 1 \\
\hline Latvia & 2 & 1 \\
\hline Ecuador & 1 & 1 \\
\hline Lithuania & 1 & 1 \\
\hline North Macedonia & 1 & 1 \\
\hline Norway & 1 & 1 \\
\hline Philippines & 1 & 1 \\
\hline Uruguay & 1 & 1 \\
\hline Azerbaijan & 3 & 0 \\
\hline Colombia & 3 & 0 \\
\hline Ethiopia & 2 & 0 \\
\hline Poland & 2 & 0 \\
\hline U Arab Emirates & 2 & 0 \\
\hline Afghanistan & 1 & 0 \\
\hline Croatia & 1 & 0 \\
\hline Ghana & 1 & 0 \\
\hline Hungary & 1 & 0 \\
\hline Israel & 1 & 0 \\
\hline Japan & 1 & 0 \\
\hline Kyrgyzstan & 1 & 0 \\
\hline Nepal & 1 & 0 \\
\hline Sri Lanka & 1 & 0 \\
\hline Tanzania & 1 & 0 \\
\hline Venezuela & 1 & 0 \\
\hline Yemen & 1 & 0 \\
& &
\end{tabular}

